\title{
A CHECKLIST OF BIRDS OF KERALA, INDIA
}

\section{Praveen J}

B303, Shriram Spurthi, ITPL Main Road, Brookefields, Bengaluru, Karnataka 560037, India paintedstork@gmail.com

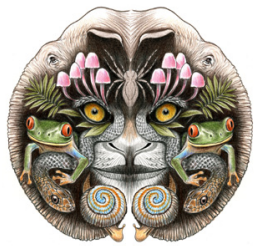

ISSN 0974-7907 (Online) ISSN 0974-7893 (Print)

\section{OPEN ACCESS}

Abstract: A checklist of birds of Kerala State is presented in this paper. Accepted English names, scientific binomen, prevalent vernacular names in Malayalam, IUCN conservation status, endemism, Wildlife (Protection) Act schedules, and the appendices in the CITES, pertaining to the birds of Kerala are also given. The State of Kerala has 500 species of birds, 17 of which are endemic to Western Ghats, and 24 species fall under the various threatened categories of IUCN.

Keywords: CITES, endemism, Malayalam name, vernacular name, Western Ghats, Wildlife (Protection) Act.

Birds are one of the better studied groups of vertebrates in Kerala. The second half of $19^{\text {th }}$ century was dotted with pioneering contributions from T.C. Jerdon, Bourdillon brothers, H.S. Ferguson, James Stewart, Rhodes Morgan and William Davison while the early half of the $20^{\text {th }}$ century included significant works of A.P. Kinloch, H.R. Baker and E.G. Phythian-Adams; all of these British workers contributed immensely to our present day ornithological knowledge. The first significant inventory of birds of Kerala was by Ferguson \& Bourdillon (1903-04) who provided an annotated checklist of 332 birds from the princely state of Travancore. However, the landmark survey of the states of Travancore and Cochin by Dr. Salim Ali in 1933-34 is widely accepted as the formal foundation in ornithology of Kerala. These surveys resulted in two highly popular books, The Birds of Travancore and Cochin (Ali 1953) and Birds of Kerala (Ali 1969); the latter listed 386 species. After two decades, Neelakantan et al. (1993) compiled information on 95 bird species that were subsequently recorded since Ali's work. Birds of Kerala - Status and Distribution (Sashikumar et al. 2011) that covered 453 species is the most recent work from the state. A checklist of birds of Kerala was also published based on this book (Sashikumar et al. 2010). As a part of this work, all records from Kerala were critically assessed and species that were included in prior publications without sufficient documentation were deleted. Praveen \&
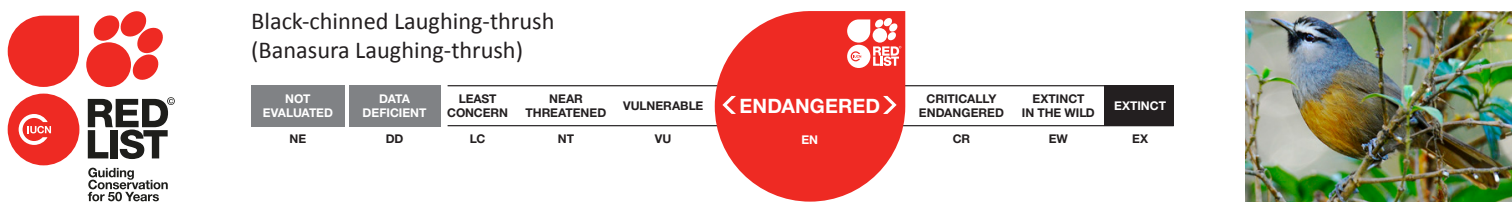

DOI: http://dx.doi.org/10.11609/jott.2001.7.13.7983-8009 | ZooBank: urn:Isid:zoobank.org:pub:ADF45BA2-D906-4D08-90DC-38B1405A9089

Editor: Mewa Singh, University of Mysore, Mysuru, India.

Date of publication: 17 November 2015 (online \& print)

Manuscript details: Ms \# 04305 | Received 11 May 2015 | Final received 23 September 2015 | Finally accepted 29 September 2015

Citation: Praveen, J. (2015). A checklist of birds of Kerala, India. Journal of Threatened Taxa 7(13): 7983-8009; http://dx.doi.org/10.11609/jott.2001.7.13.7983-8009

Copyright: (C) Praveen 2015. Creative Commons Attribution 4.0 International License. JoTT allows unrestricted use of this article in any medium, reproduction and distribution by providing adequate credit to the authors and the source of publication.

Funding: None.

Conflict of Interest: Vernacular names used in this MS has been directly taken from an online directory maintained by Birdwatchers of Kerala Facebook group with explicit permission from its moderator.

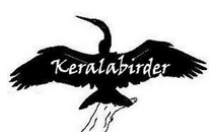

Acknowledgements: I would like to acknowledge the contribution of my team members viz., P.O. Nameer, Rajeev Raghavan, A. Bijukumar, Mohamed Jafer Palot and Sandeep Das in getting this checklist into a final shape. Dileep Anthikkad, Sandeep Das and Vivek Chandran contributed the images used along with this paper. I would also like to acknowledge help rendered by Jinesh P.S. of Birdwatchers of Kerala Facebook group in finalising the vernacular names of birds of Kerala. My thanks are due to two anonymous referees for correcting several errors in this paper. 
Narayanan (2013) updated this checklist with 32 more species recorded between May 2010 and December 2013. They additionally listed eight species which have been historically recorded from Kerala with definite evidence, but not recorded since 1 January 1975. Since the publication of Praveen \& Narayanan (2013), eight species have been added to the avifauna of Kerala through formal publications. These cases are annotated with footnotes. Though naturalised species like Rock Pigeon Columba livia are included in the checklist, definite or potential cage escapees like Red-breasted Parakeet Psittacula alexandri, Budgerigar Melopsittacus undulatus and Blue-throated Bee-eater Merops viridis are excluded. Acute identification difficulties exist in some cases for sight and photographic records particularly for Riparia martins where field separation is impossible on current knowledge. Hence, Pale Martin Riparia diluta and Sand Martin R. riparia are excluded from the list (Praveen \& Narayanan 2014) though definitely one of the species has been reported multiple times from Kerala. Pelagic birds that occur within the maritime limits of Kerala are marked in blue.

Avian taxonomy is in a lot of flux in the last decade with genetic studies questioning traditional taxonomy. We followed the taxonomy of Howard \& Moore $4^{\text {th }}$ edition (Dickinson \& Remson 2013; Dickison \& Christidis 2014) putting a premium on stability over informed speculation. This would mean that several splits proposed by recent authors (Rasmussen \& Anderton 2012) and uncritically accepted by other global taxonomies (Gill \& Donskar 2014; Clements et al. 2014) are not considered here as these proposals are conjectures awaiting separate taxonomic treatment. Birdlife International (2014) follows taxonomy based on the HBW and BirdLife International Illustrated Checklist of the Birds of the World (delHoyo \& Collar 2014); however, the second part on passerines, which essentially has encountered major upheavals, is yet to be assessed. However, there is definitely a scope that most of these taxonomic proposals are likely to be supported in the future with molecular evidence. Hence, we provide the details of contradicting taxonomies as footnotes. In the context, it is worthwhile to state that we follow Praveen et al. (2014) for English names. This reference essentially follows Howard \& Moore $4^{\text {th }}$ edition for taxonomy but have adapted the English names that are more widely used in India (e.g., Yellow-throated Sparrow for Chestnut-shouldered Bush Sparrow). Alternate English names that are prevalent in India for these birds are included in brackets.

Birds probably have the most extensive vernacular literature in Kerala. Names proposed by Neelakantan
(1984) and later extended by Grimmett et al. (2007) and Sashikumar et al. (2011) are generally accepted. Alternate names prevalent in other parts of Kerala are also included. The complete compilation of Malayalam names for all the species were posted in the Birdwatchers of Kerala community in social media (Facebook) and several feedbacks and suggestions obtained are incorporated. BirdLife International has published the 2015 revision of redlist for birds and the same has been used in this work.

In this monograph, 500 species of birds in 88 families and 22 orders are listed. Out of which 17 are endemic to Western Ghats. Twenty-five species fall under the various threatened categories of IUCN, 32 are Near Threatened, 443 fall under one of the schedules of Wildlife (Protection) Act and 71 fall under one of the appendices of CITES.

\section{REFERENCES}

Ali, S. (1953). The Birds of Travancore and Cochin. Oxford University Press, Oxford, 436pp.

Ali, S. (1969). Birds of Kerala. Oxford University Press, Oxford, 444pp.

Bhat, A.K. (2014) From the field:Birds:Long-tailed Skua Stercorarius longicaudus from Azheekkal coast, Kannur District. Malabar Trogon 12(1-3): 36.

BirdLife International (2014). The BirdLife Checklist of the Birds of the World: Version 7. Downloaded from http://www.birdlife.org/ datazone/userfiles/file/Species/Taxonomy/BirdLife_Checklist_ Version_70.zip [.xls zipped $1 \mathrm{MB}$ ]

Clements, J.F., T.S. Schulenberg, M.J. Iliff, M.J., D. Roberson, T.A. Fredericks, B.L. Sullivan \& C.L. Wood (2014). The eBird/Clements checklist of birds of the world: Version 6.9. Downloaded from http:// www.birds.cornell.edu/clementschecklist/download/

Damle, S. \& T. Inskipp (2014). Sighting of Northern Wheatear Oenanthe oenanthe from the Nubra Valley, Ladakh, Jammu and Kashmir, and a review of previous records from the Indian Subcontinent. Indian Birds 9(5\&6): 139-141

del Hoyo, J. \& N.J. Collar (2014). HBW and BirdLife International Illustrated Checklist of the Birds of the World. Volume 1: NonPasserines. Lynx Edicions, Barcelona, 903pp.

Dickinson, E.C. \& J.V.J Remsen (eds.) (2013). The Howard and Moore Complete Checklist of the Birds of the World: 1. Non-Passerines. 4th edition. Eastbourne, U.K.: Aves Press. Vol. 1 of 2 vols, 461pp.

Dickinson, E.C. \& L. Christidis (eds.) (2014). The Howard and Moore Complete Checklist of the Birds of the World: Vol. 2. Passerines. 4th ed. Eastbourne, U.K.: Aves Press. Vol. 2 of 2 vols. 752pp, plus CD content.

Ferguson, H.S. \& T.F. Bourdillon (1903-04). The birds of Travancore, with notes on their nidification. Part I-III. Journal of the Bombay Natural History Society. XV (2): 249-264, XVI(1): 1-18, XV:(3): 455474, XV(4): 654-673

Gandhe, A. (2014). Seabird observations off the western coast of India. Indian Birds 9(5\&6): 137-138.

Gill, F. \& D. Donsker (eds.). (2014). IOC World Bird List (v 4.3); http:// dx.doi.org/10.14344/IOC.ML.4.3

George, P.J. (2014). From the field: Birds: Tree Pipit Anthus trivialis from Attappadi, Palakkad Distrct. Malabar Trogon 12 (1-3): 36

Grimmett, R., T. Inskipp \& P.O. Nameer (2007). Thekkeindiayile Pakshikal [Birds of southern India]. BNHS Field Guides. [In Malayalam]. A. C. Black. London, 1-240pp.

Karuthedathu, D., D. James, A. Sasidevan, M. Moghul, P. Parab, S.S. 


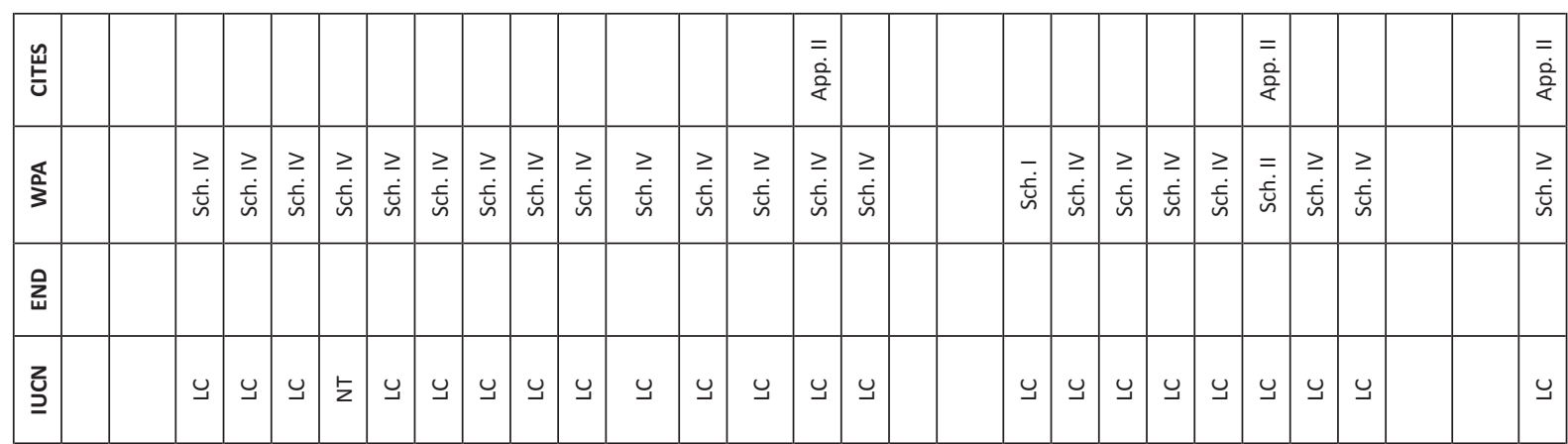

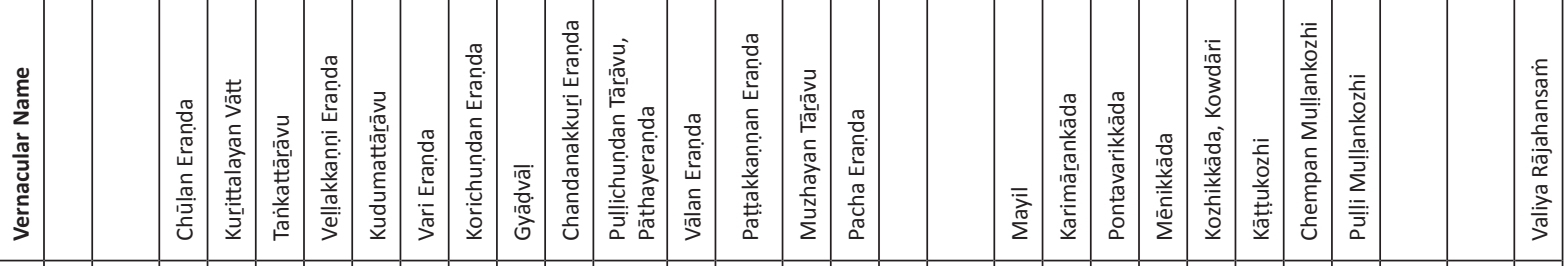

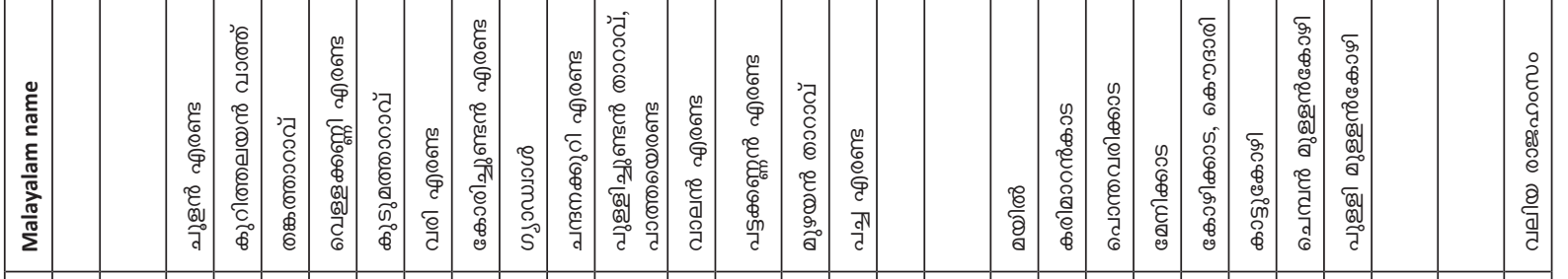

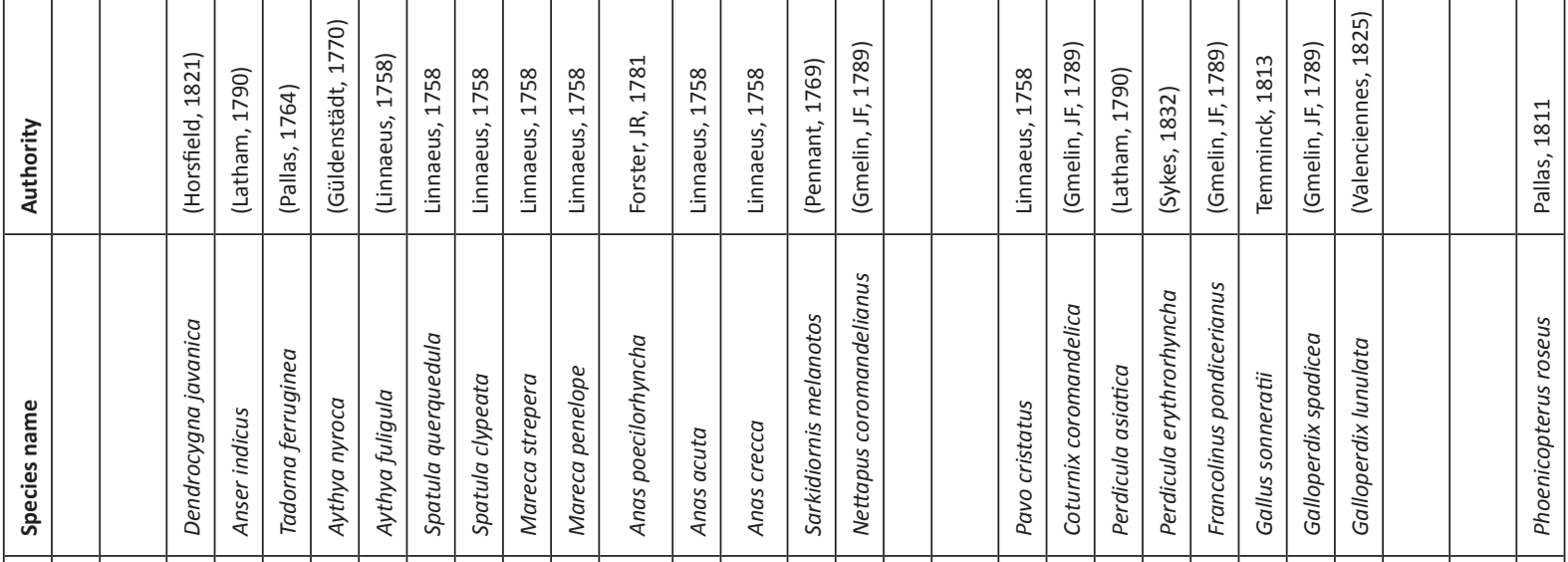

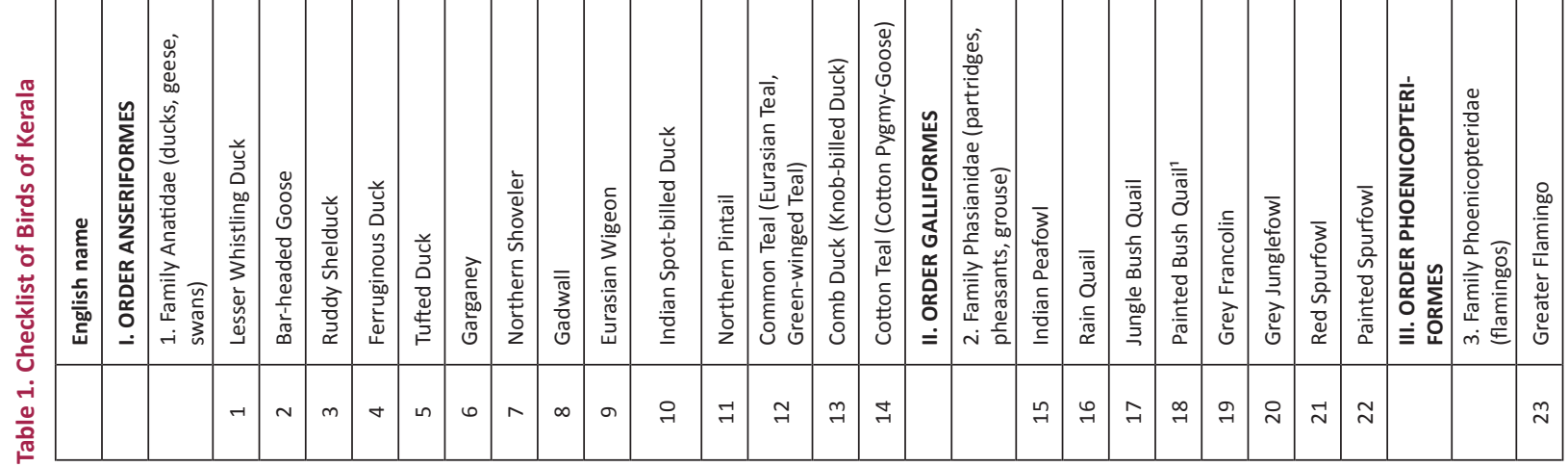




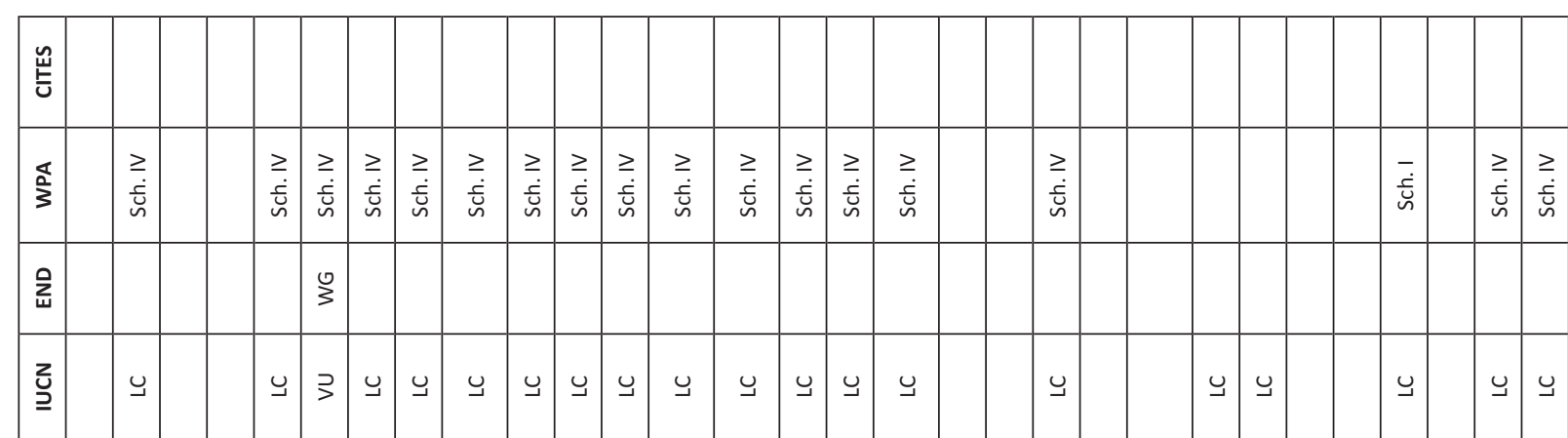

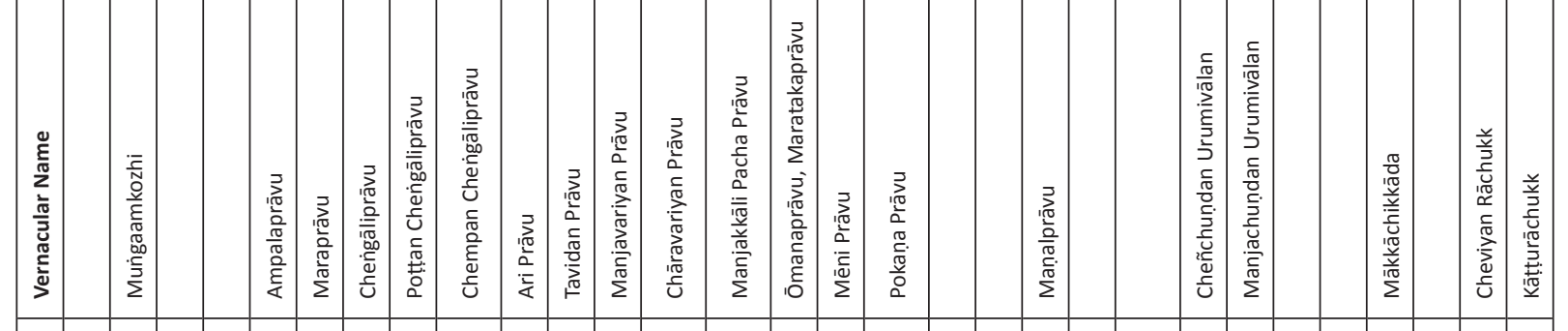

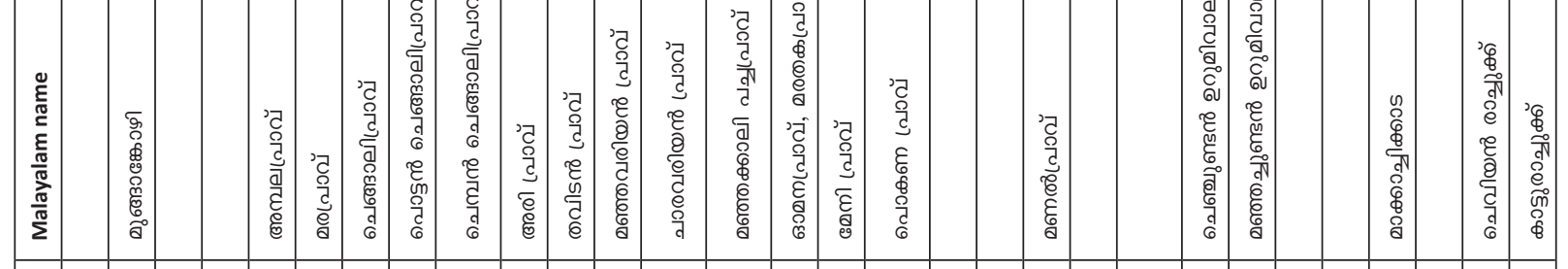

\begin{tabular}{|c|c|c|c|c|c|c|c|c|c|c|c|c|c|c|c|c|c|}
\hline 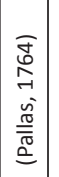 & 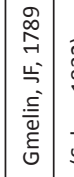 & 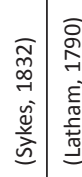 & 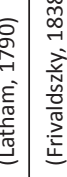 & 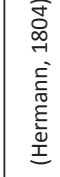 & 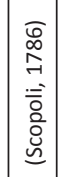 & 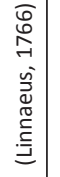 & 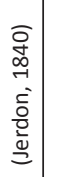 & 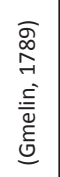 & 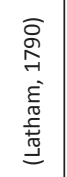 & 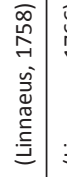 & & 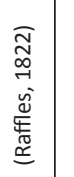 & 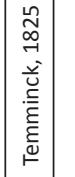 & & 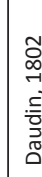 & & 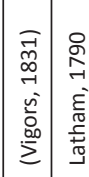 \\
\hline
\end{tabular}

\begin{tabular}{|c|c|c|c|c|c|c|c|c|c|c|c|c|c|c|c|c|}
\hline 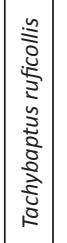 & 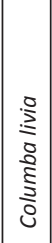 & 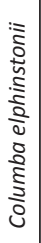 & & & & 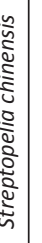 & 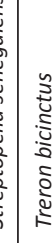 & 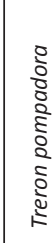 & 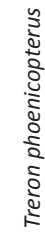 & 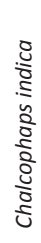 & 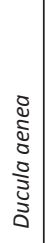 & 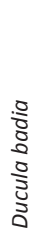 & 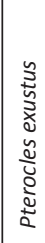 & 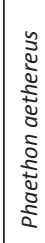 & 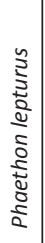 & 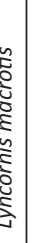 \\
\hline
\end{tabular}

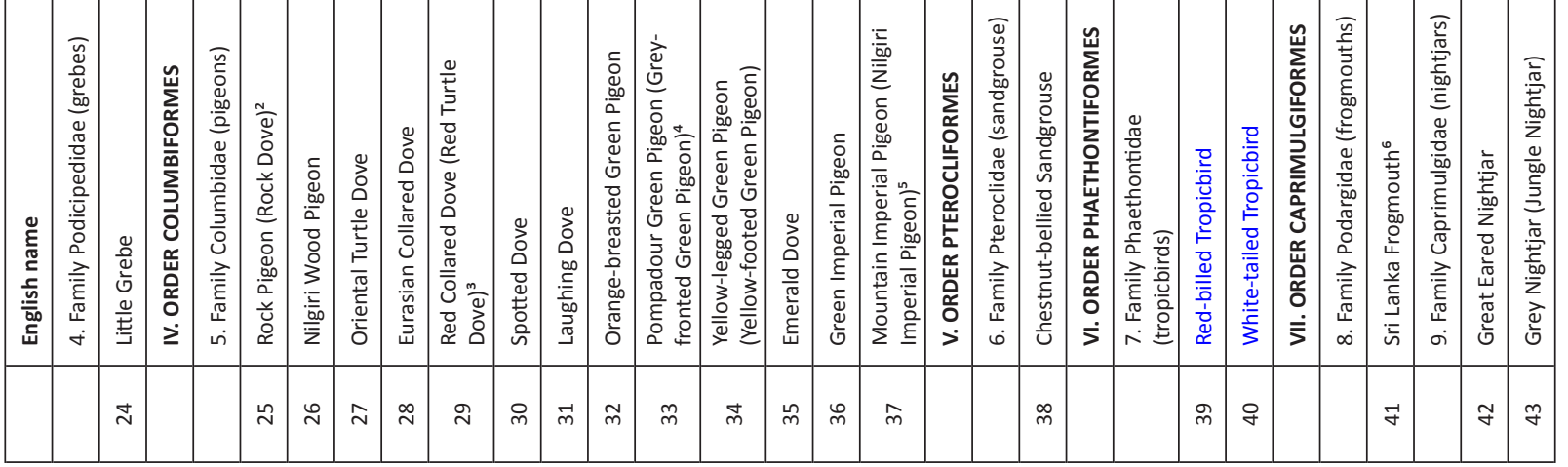




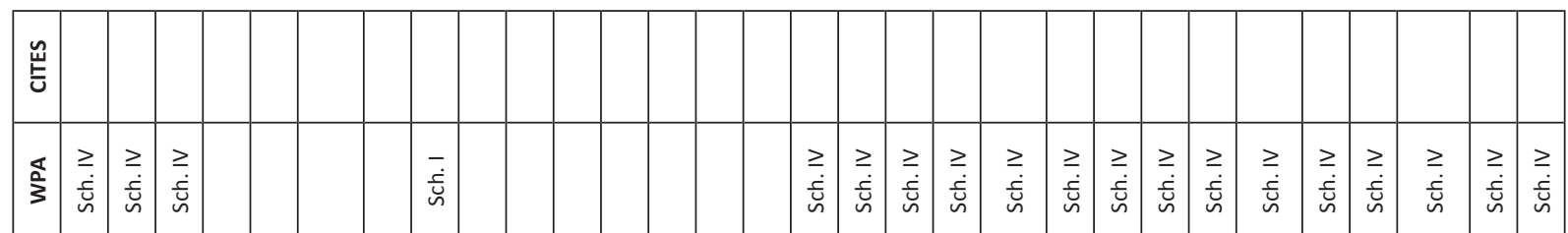

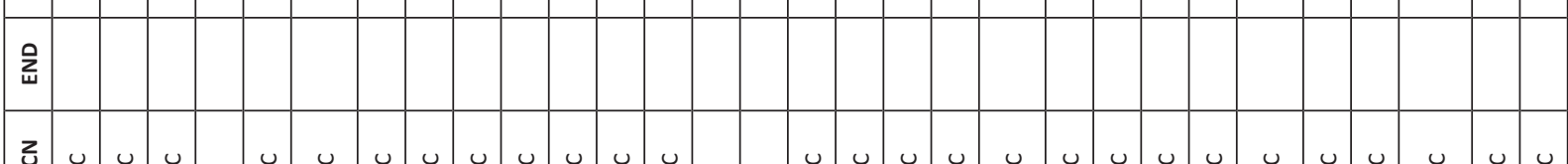

こ

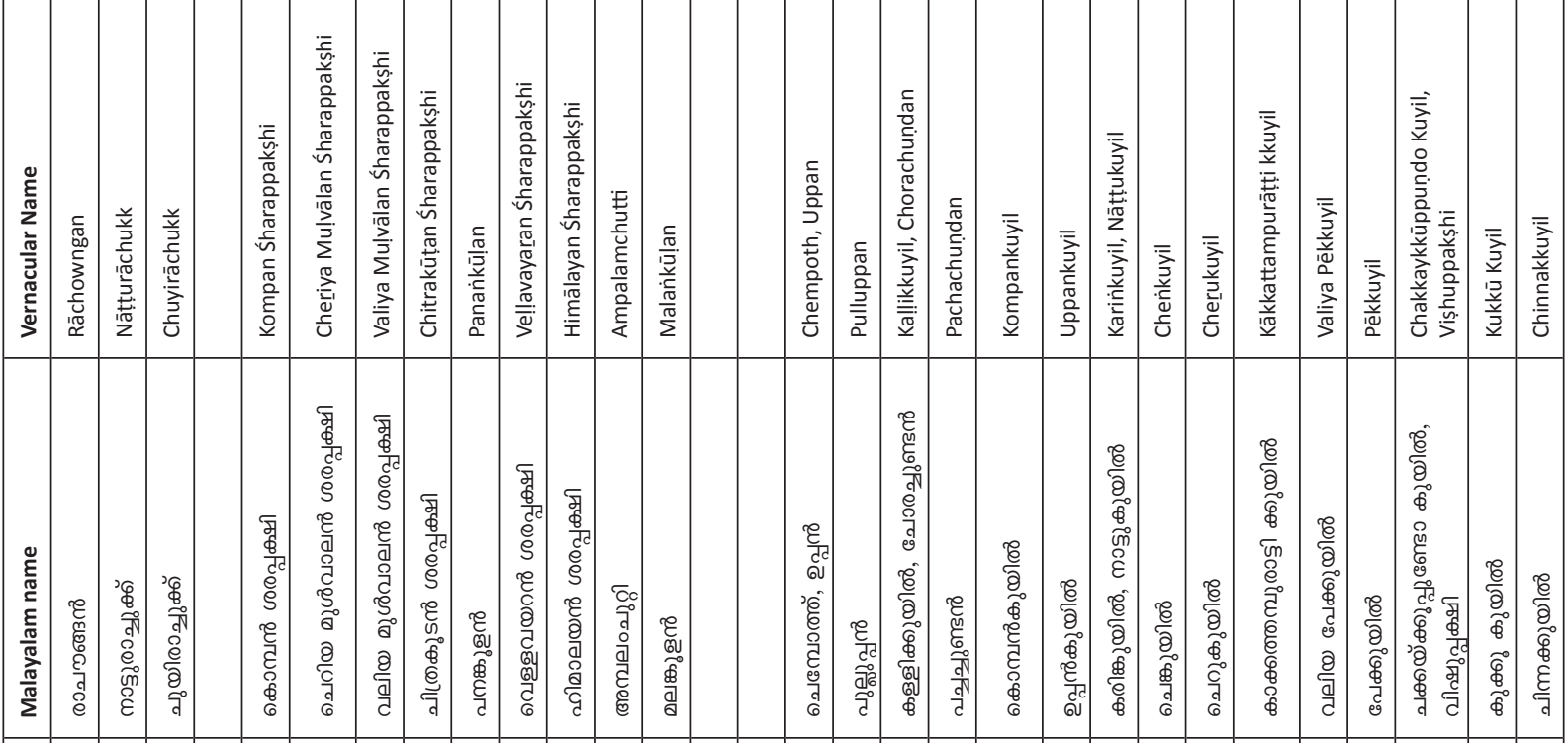

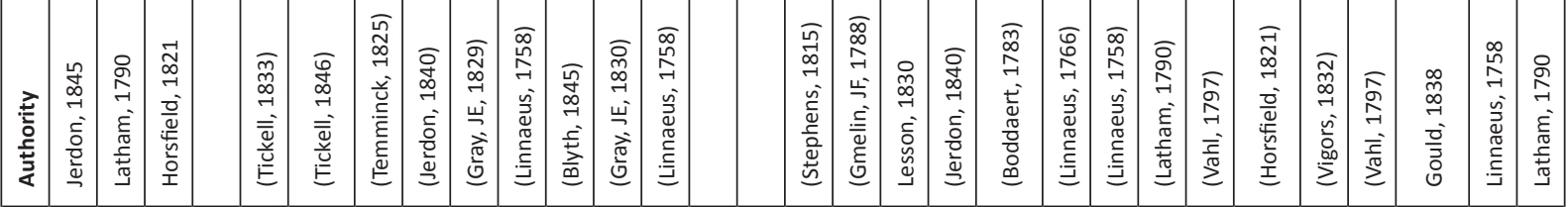

\begin{tabular}{|c|c|c|c|c|c|c|c|c|c|c|c|c|c|c|c|c|c|c|c|c|c|c|}
\hline 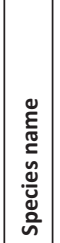 & 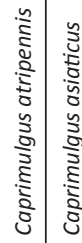 & 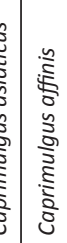 & 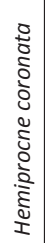 & 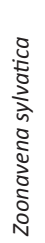 & 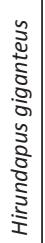 & 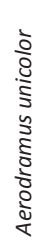 & 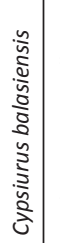 & 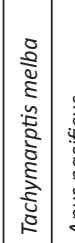 & 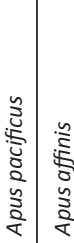 & $\begin{array}{l}3 \\
\frac{3}{2} \\
\frac{3}{8}\end{array}$ & 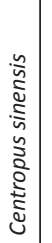 & 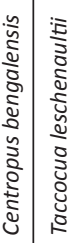 & 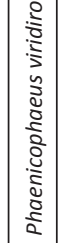 & 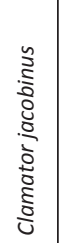 & 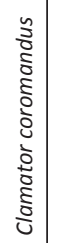 & 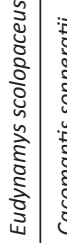 & 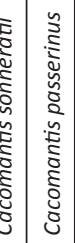 & 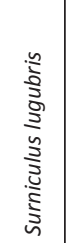 & 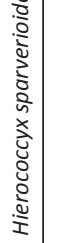 & 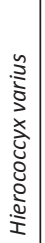 & 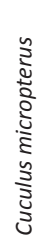 & 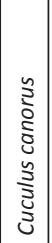 \\
\hline
\end{tabular}

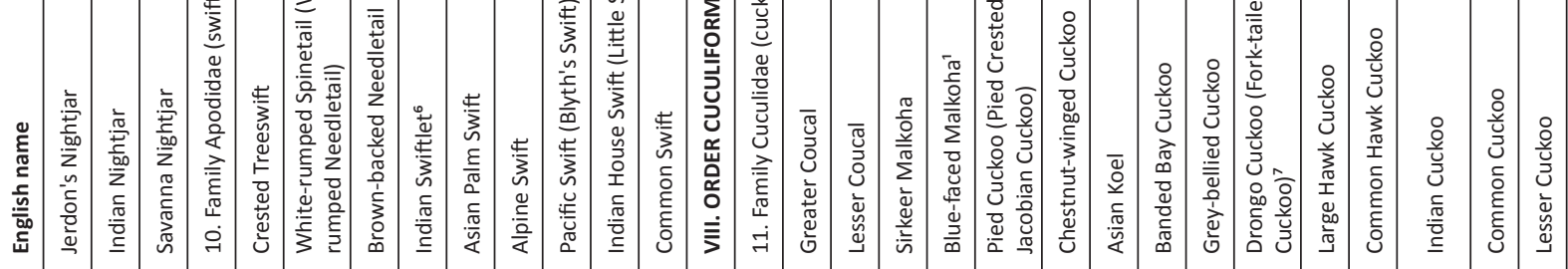
\& 


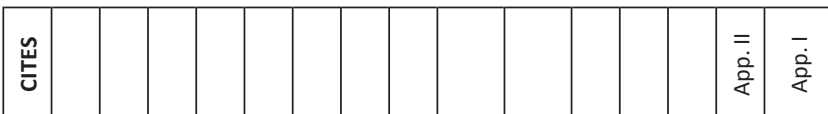

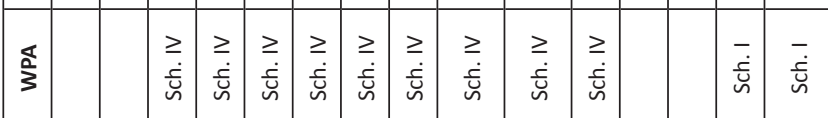

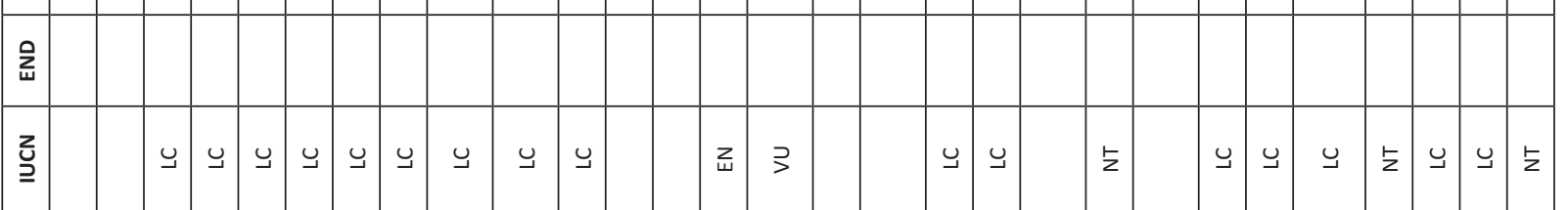

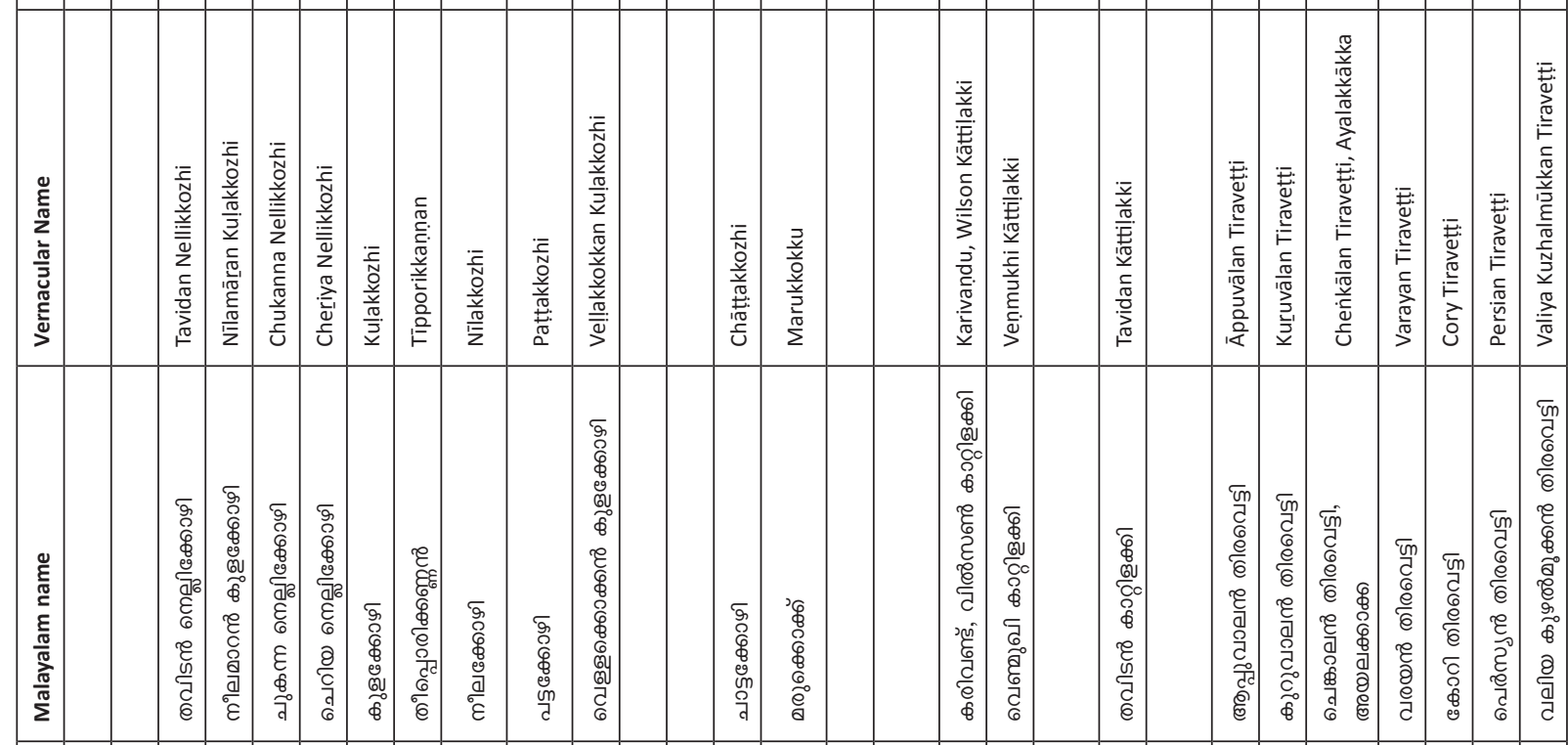

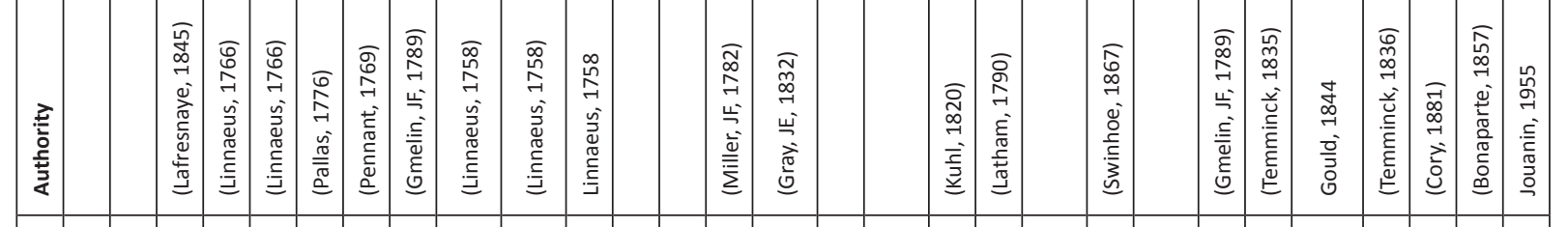

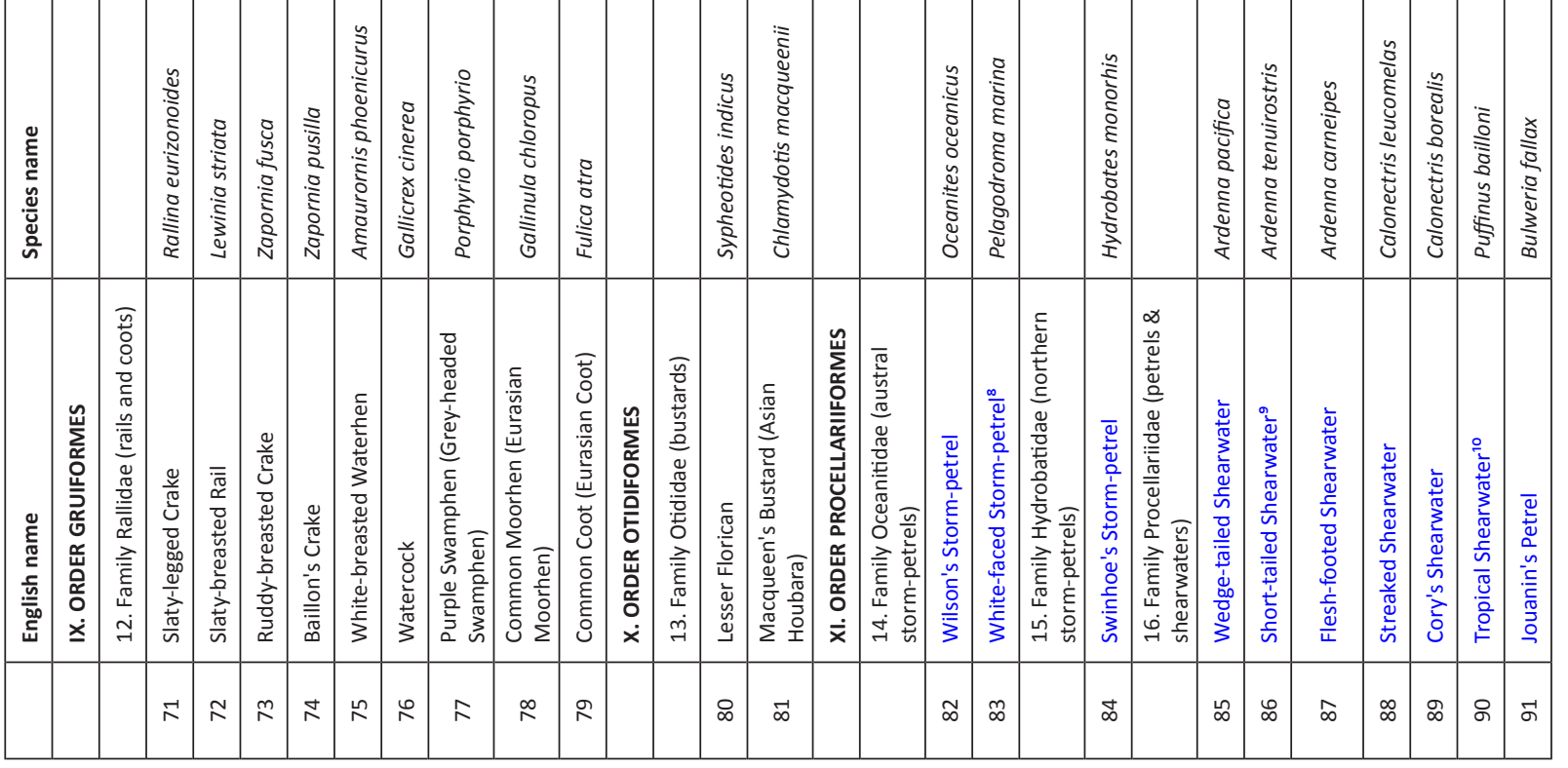




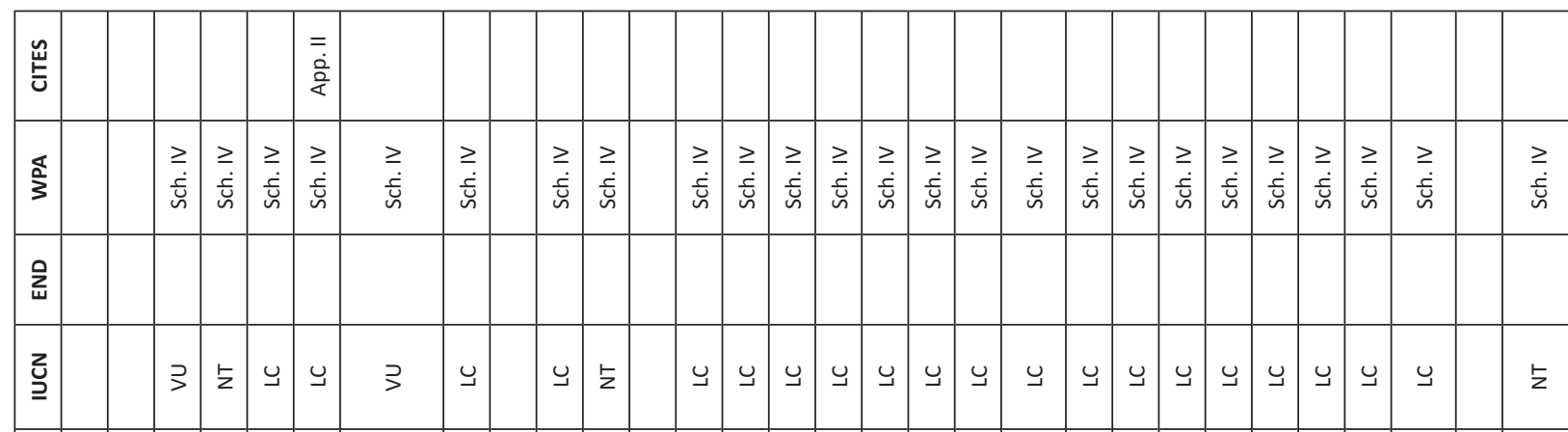

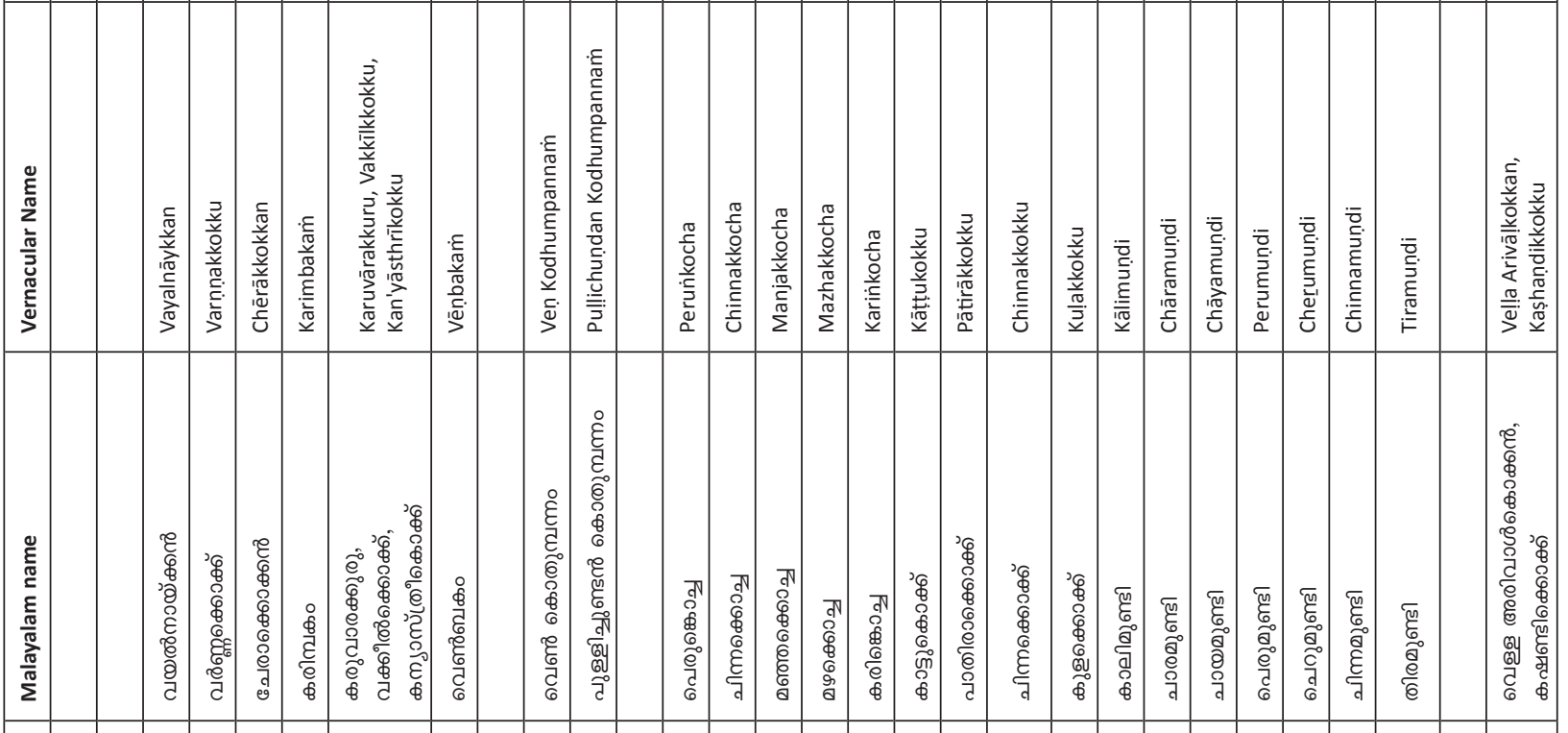

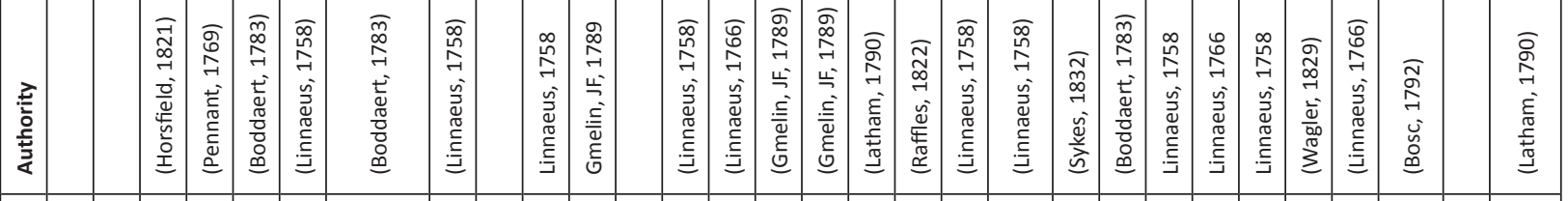

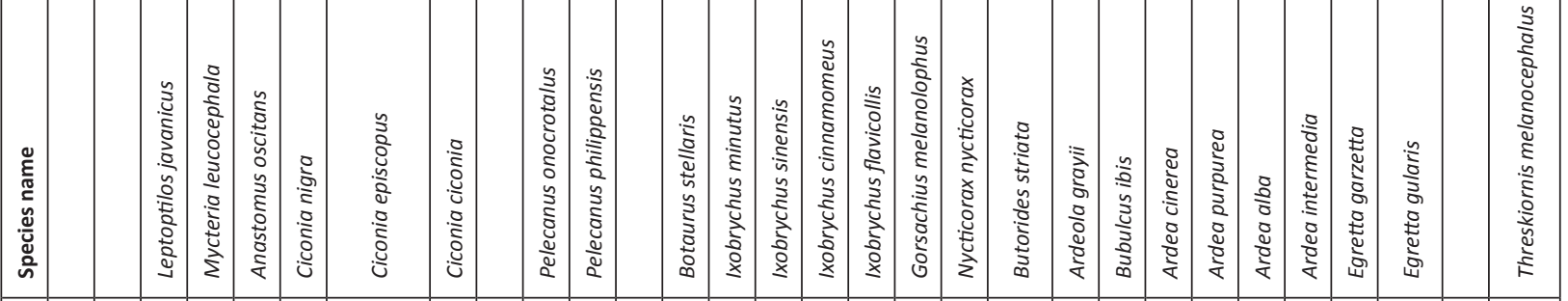

\begin{tabular}{|c|c|c|c|c|c|c|c|c|c|c|c|c|c|c|c|c|c|c|c|c|c|c|c|c|}
\hline \multirow[t]{2}{*}{ 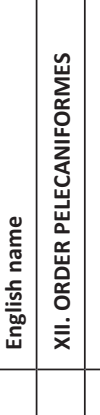 } & 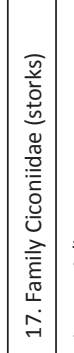 & 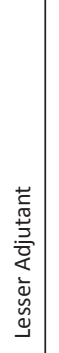 & 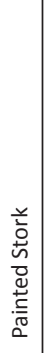 & & & \multirow{2}{*}{\multicolumn{2}{|c|}{ 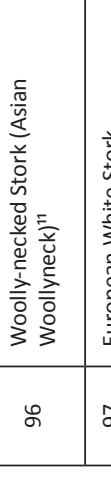 }} & \multirow[t]{2}{*}{ 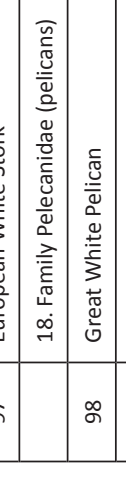 } & & \multirow[t]{2}{*}{ 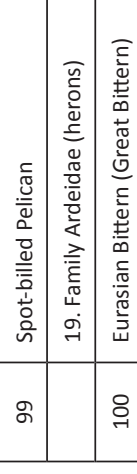 } & 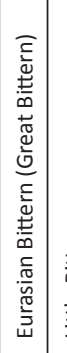 & \multirow{2}{*}{\multicolumn{2}{|c|}{ 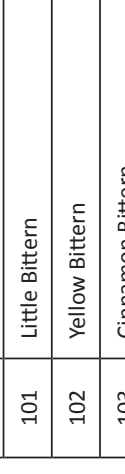 }} & \multirow{2}{*}{\multicolumn{2}{|c|}{ 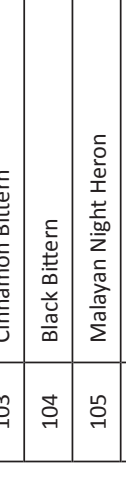 }} & \multirow{2}{*}{ 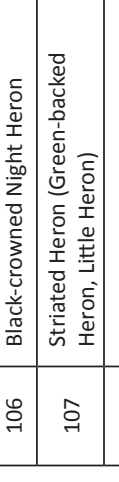 } & 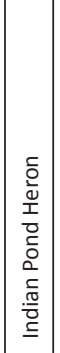 & \multirow[t]{2}{*}{ 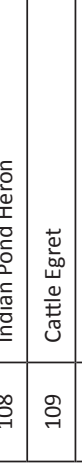 } & \multirow[t]{2}{*}{ 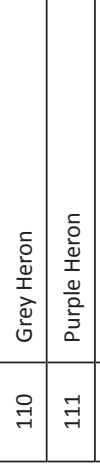 } & \multirow{2}{*}{\multicolumn{2}{|c|}{ 悹 }} & \multirow{2}{*}{\multicolumn{2}{|c|}{ 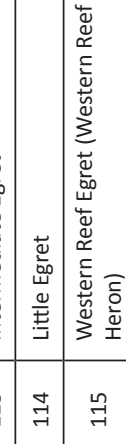 }} & | \\
\hline & & $\tilde{\sigma}$ & $\curvearrowleft$ & \% & & & & & $\infty$ & & ৪) & & & & & & $\stackrel{\infty}{\sim}$ & & & & & & & \\
\hline
\end{tabular}




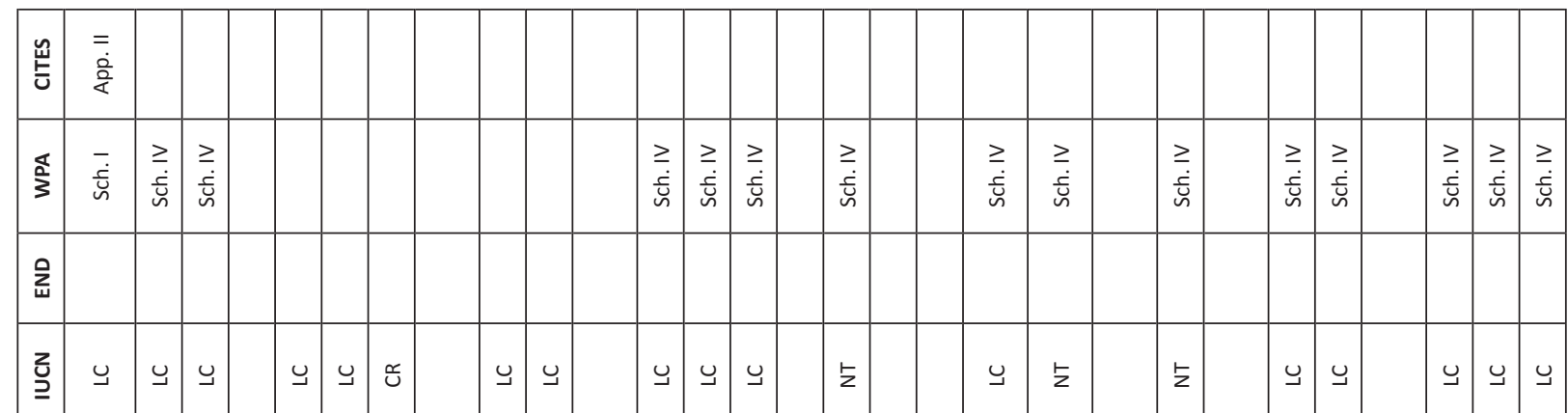

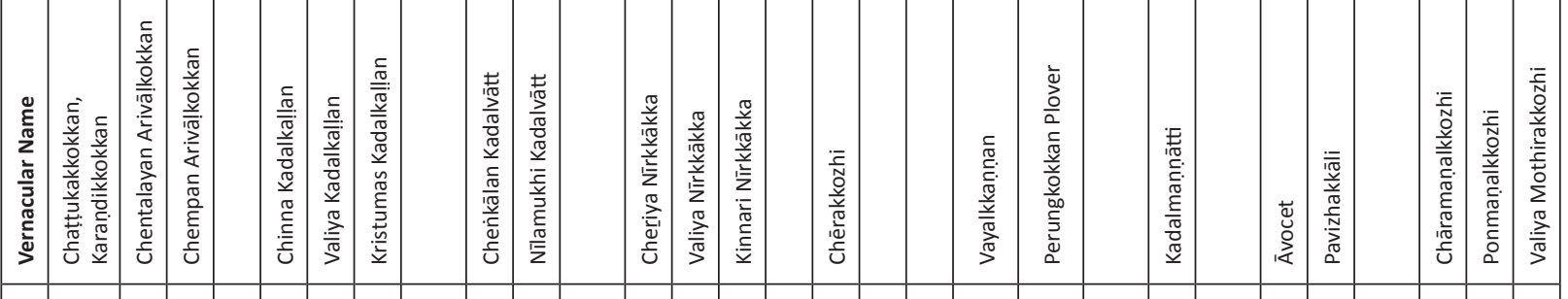

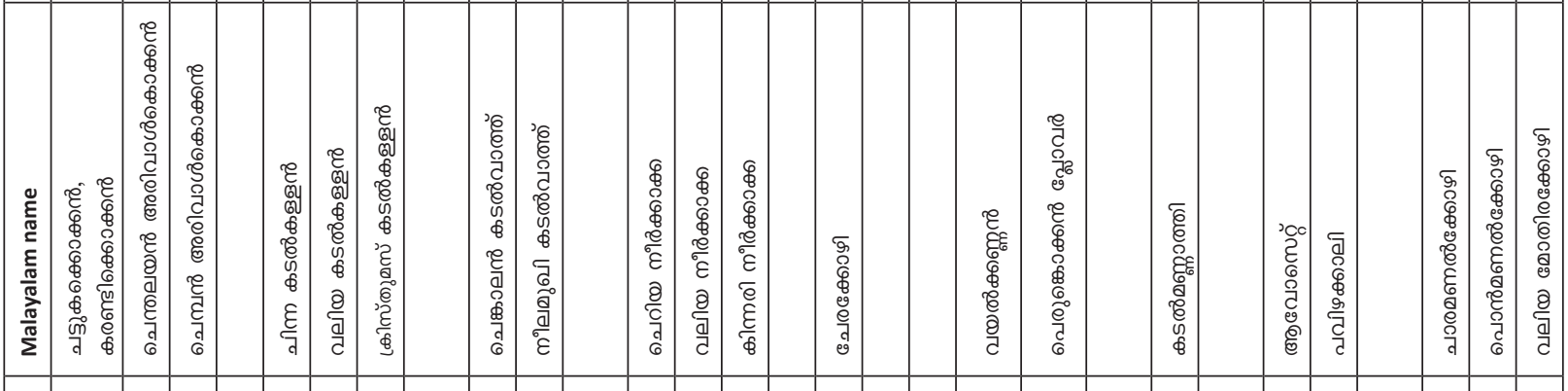

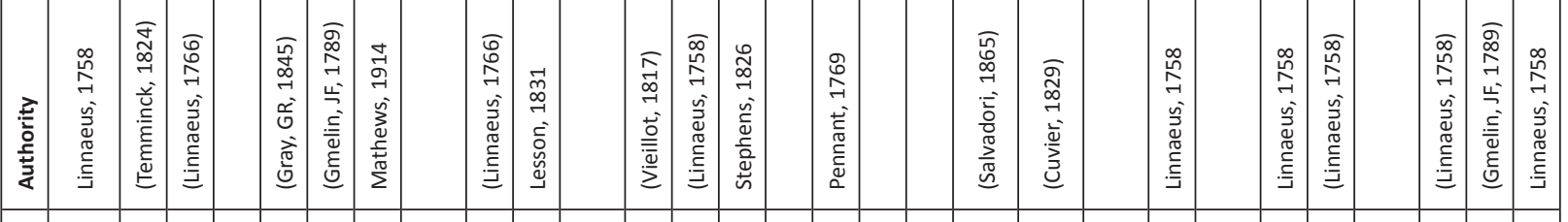

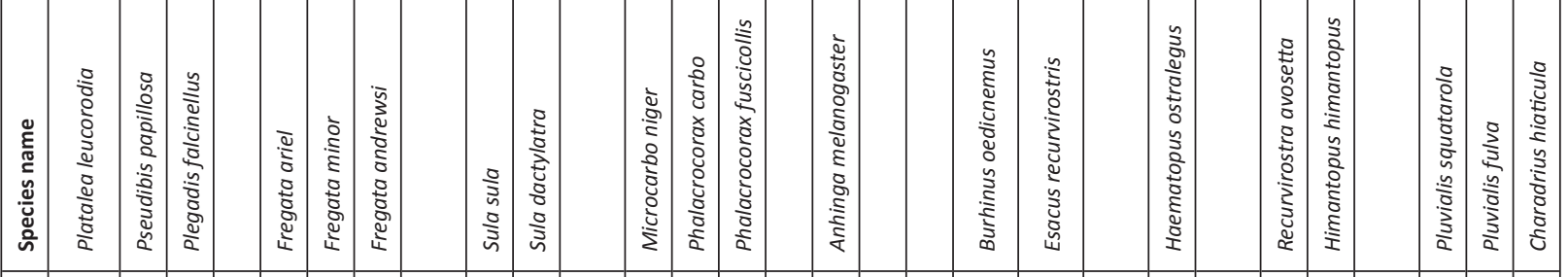

\begin{tabular}{|c|c|c|c|c|c|c|c|c|c|c|c|c|c|c|c|c|c|c|c|c|c|c|c|}
\hline 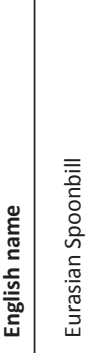 & 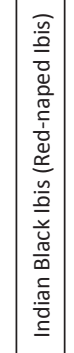 & 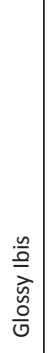 & 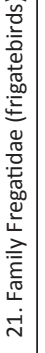 & 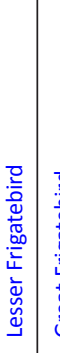 & 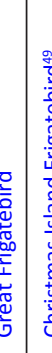 & 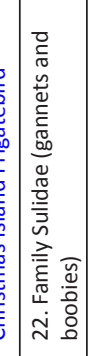 & 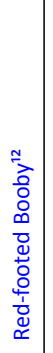 & 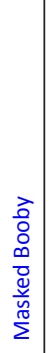 & 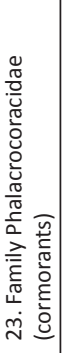 & 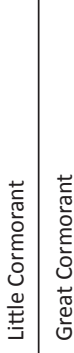 & 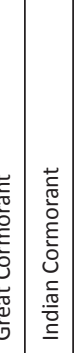 & 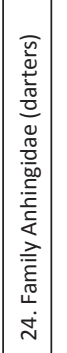 & 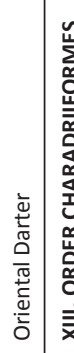 & 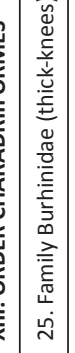 & 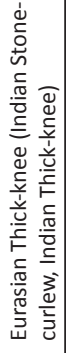 & 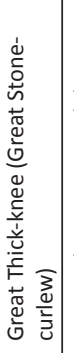 & 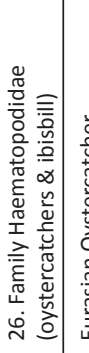 & 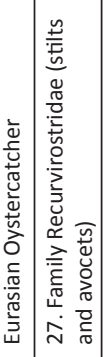 & 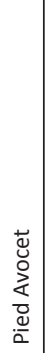 & 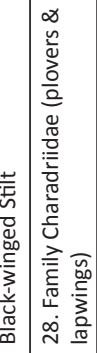 & 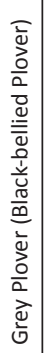 & 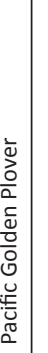 & \\
\hline$\exists$ & $\stackrel{\infty}{\underset{\sim}{*}}$ & $\underset{7}{7}$ & & 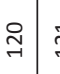 & 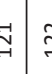 & & $\underset{\widetilde{\exists}}{\tilde{\exists}}$ & $\underset{\mathbb{Z}}{\mathbb{Z}}$ & & 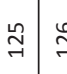 & 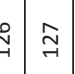 & & $\stackrel{\infty}{\underset{\exists}{\sim}}$ & & స్ & 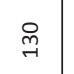 & & $\overrightarrow{\ddot{m}}$ & $\underset{\sim}{\tilde{m}}$ & m్ & $\underset{\sim}{ \pm ্}$ & 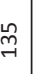 & \\
\hline
\end{tabular}




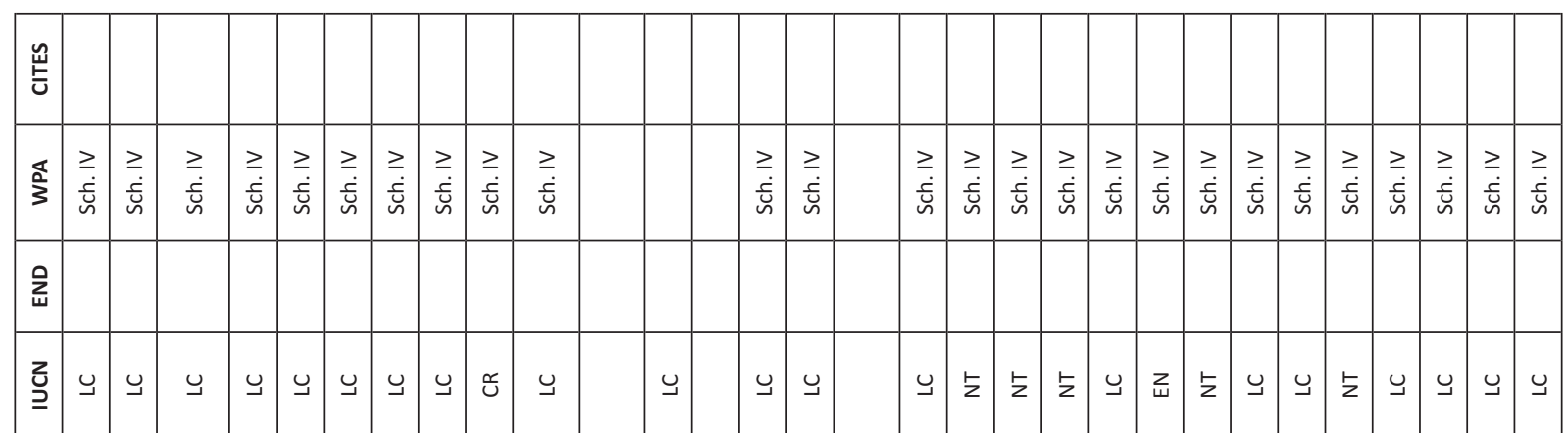

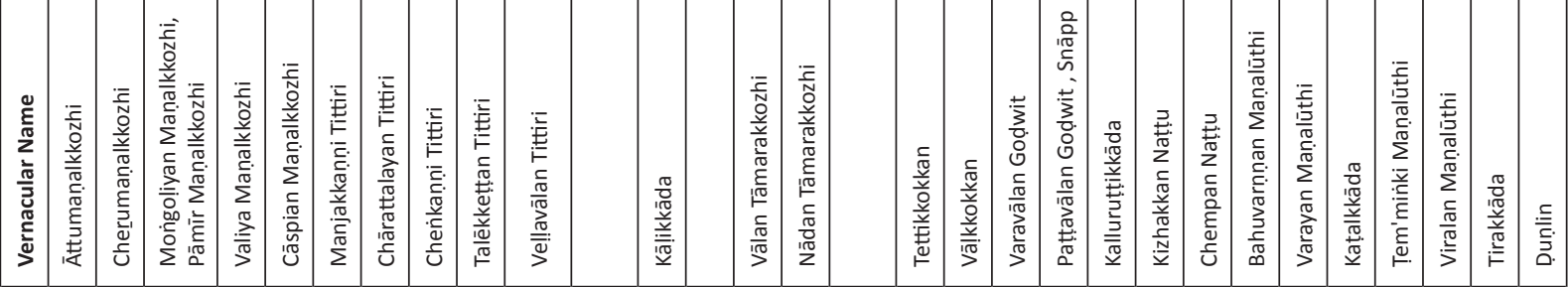

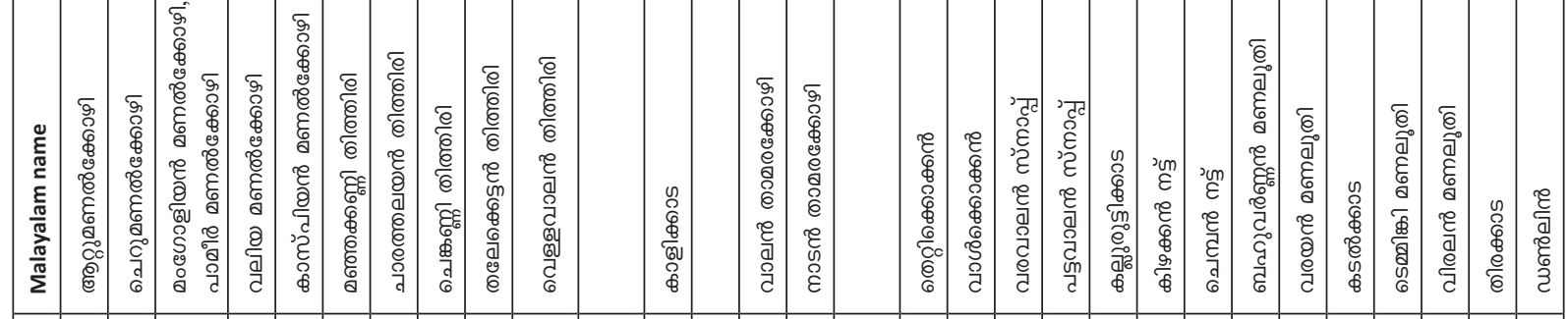

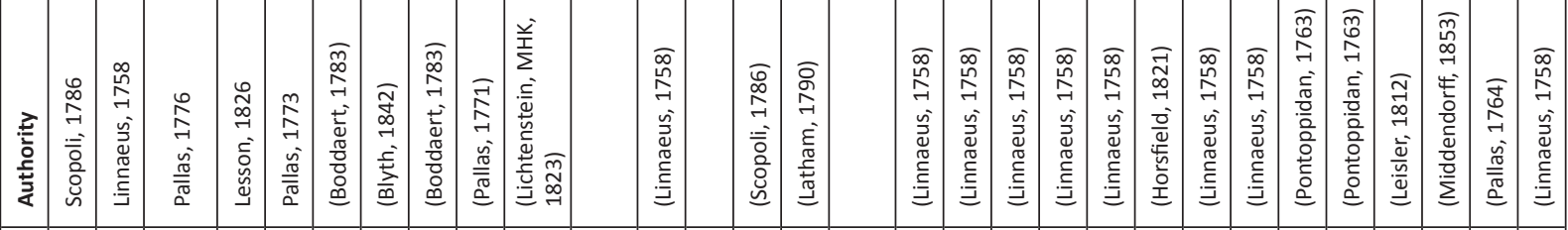

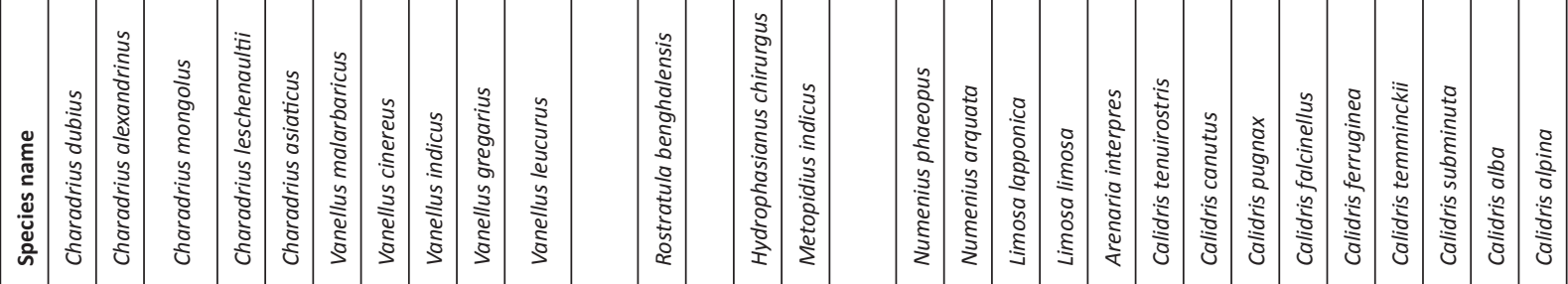

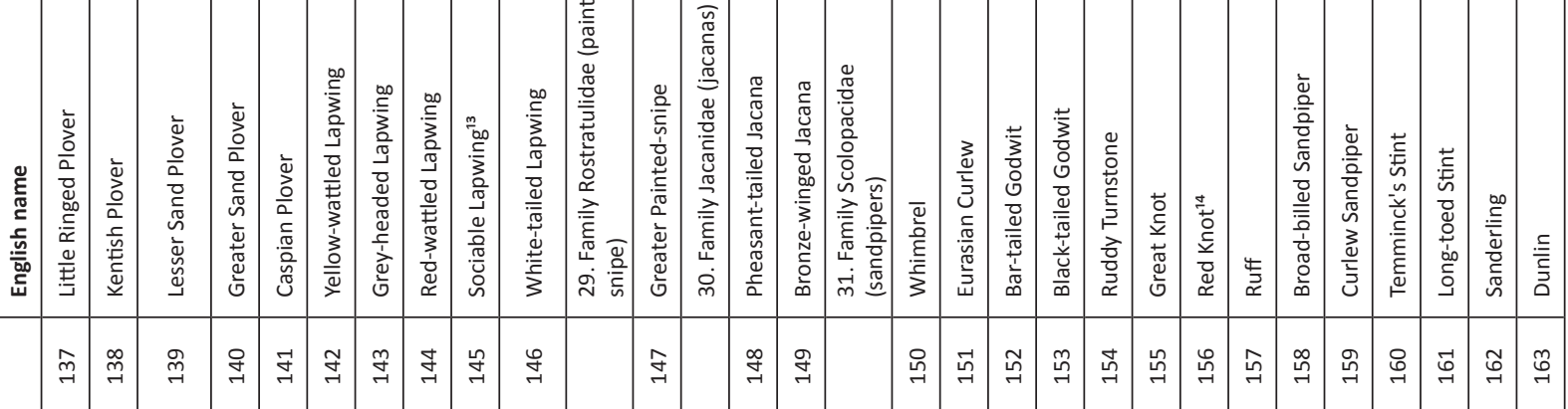




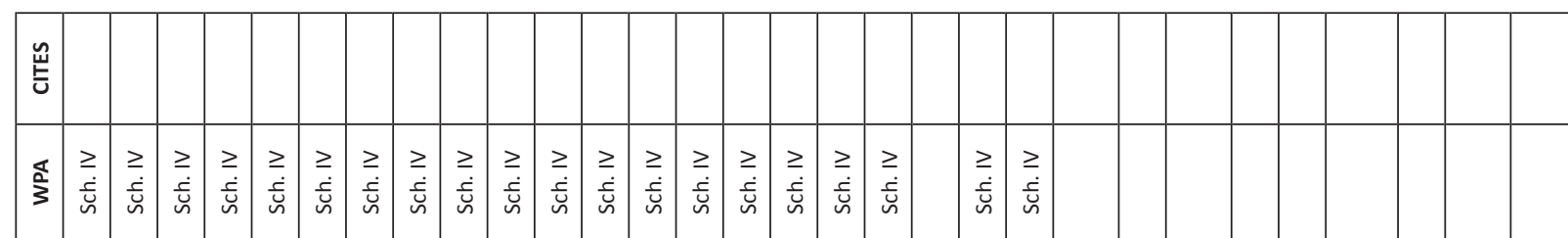

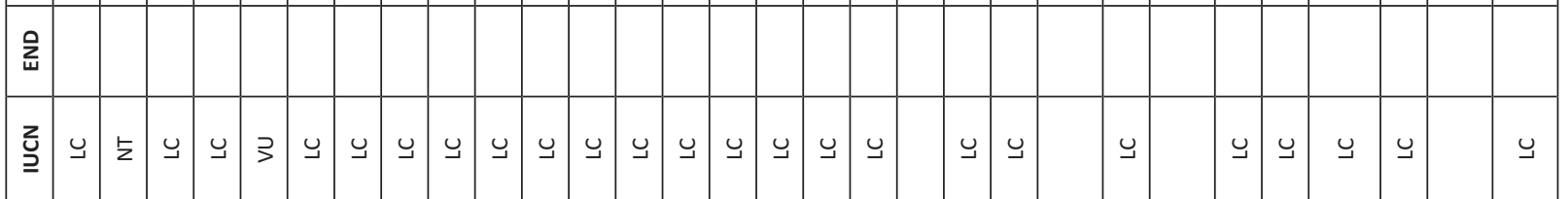

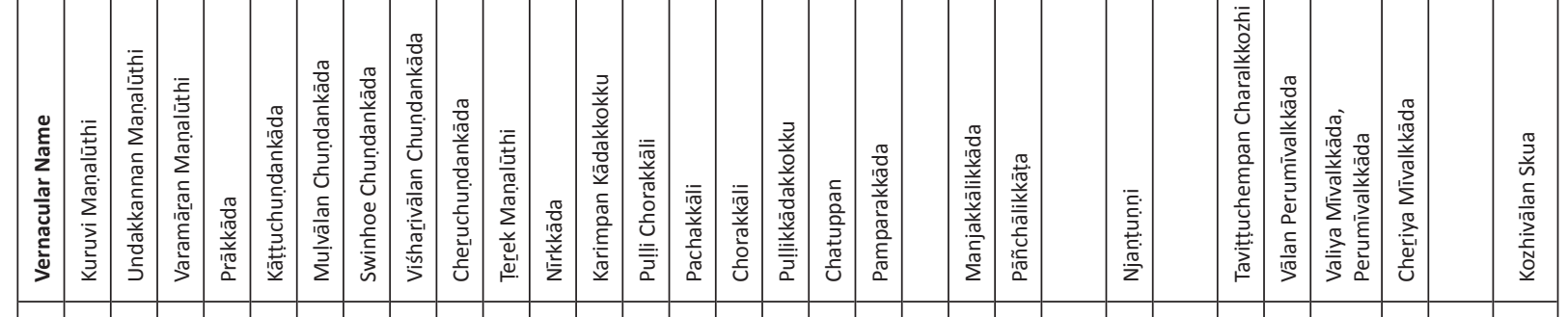

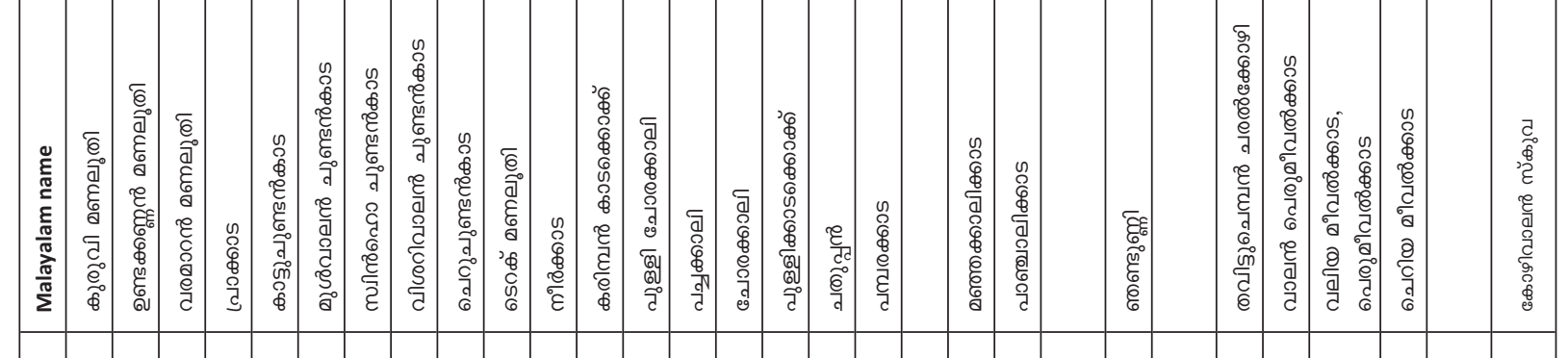

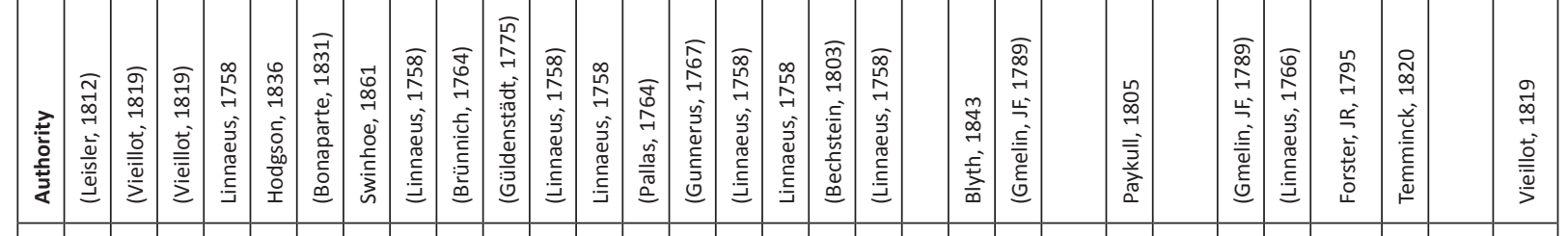

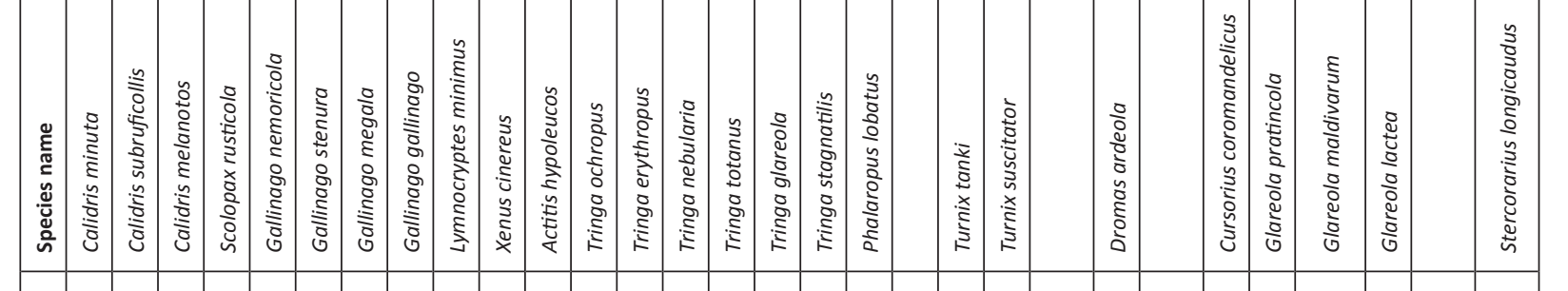

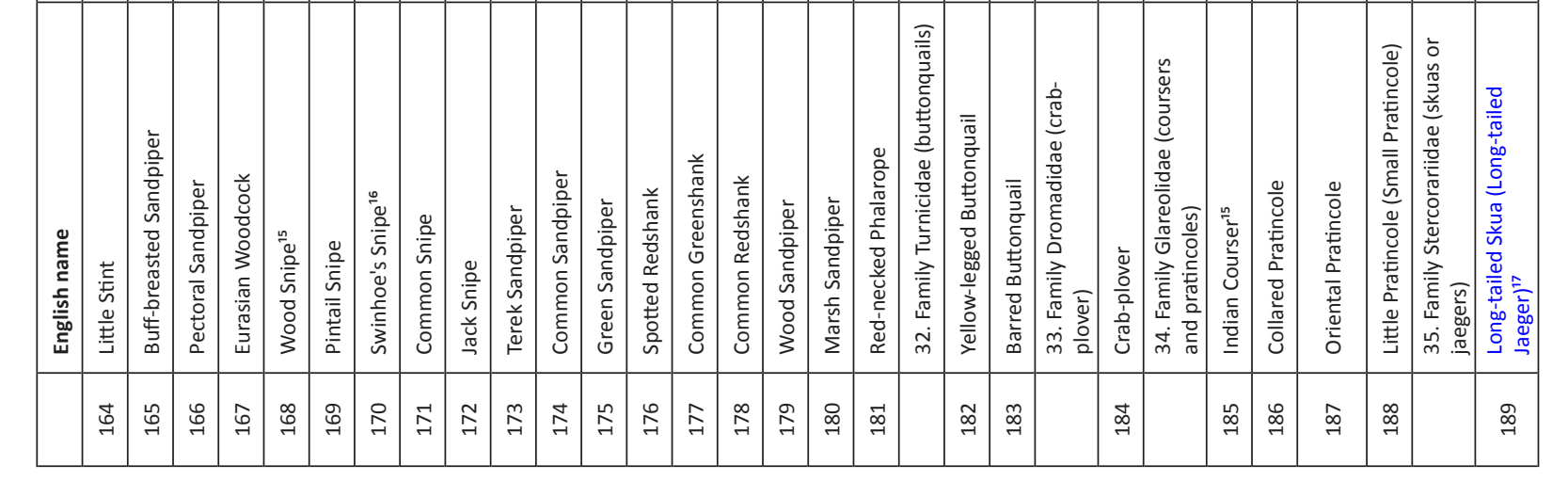




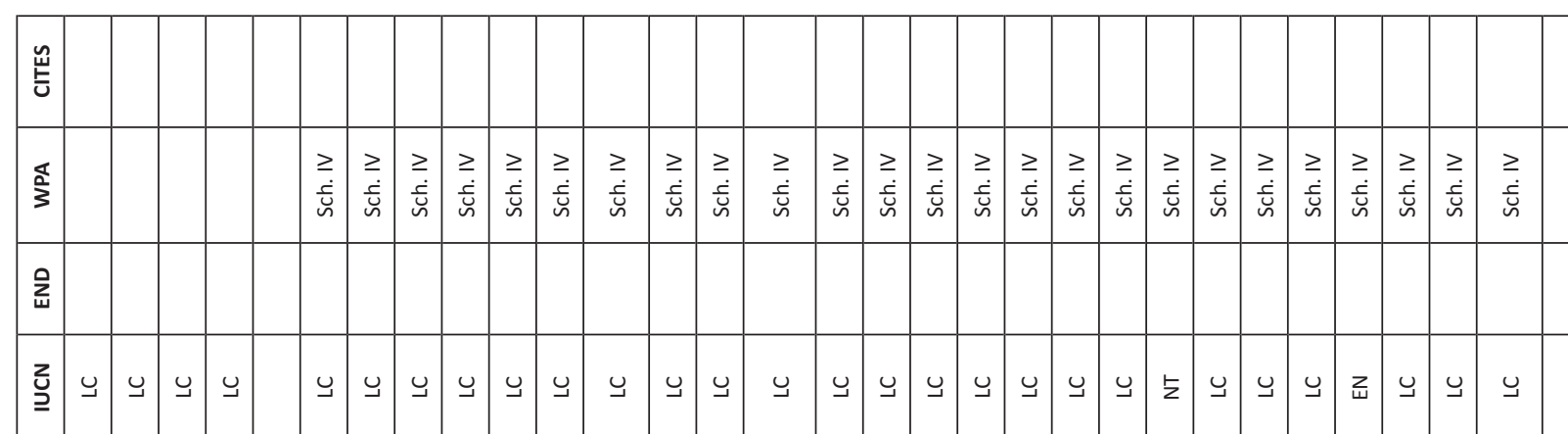

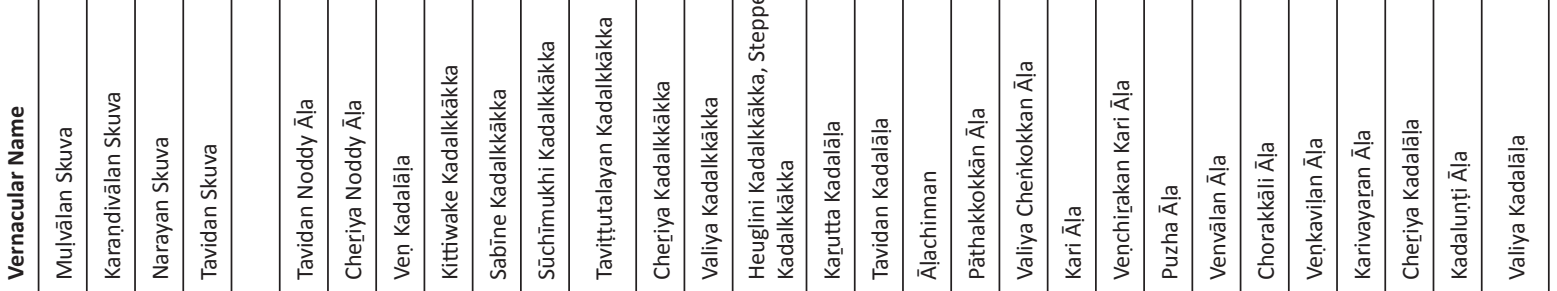

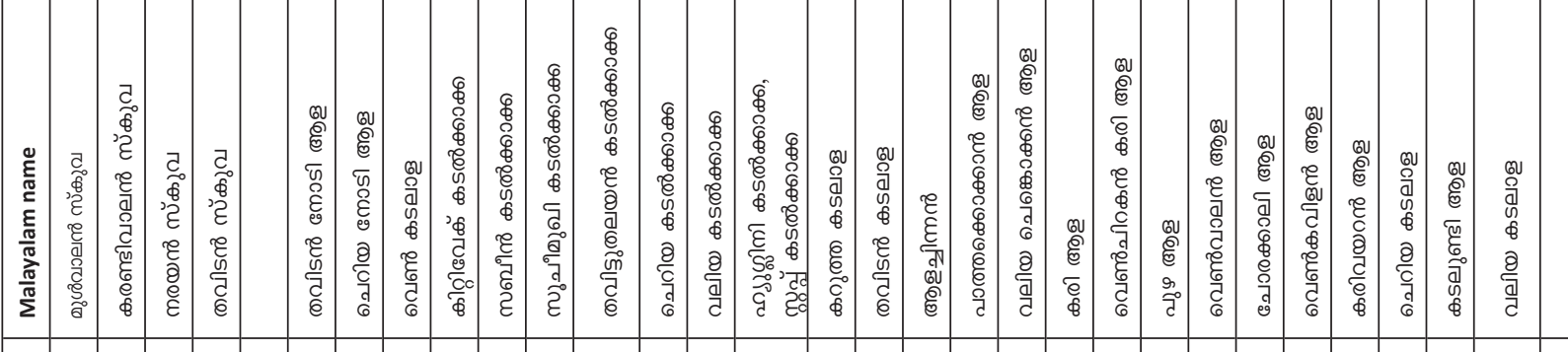

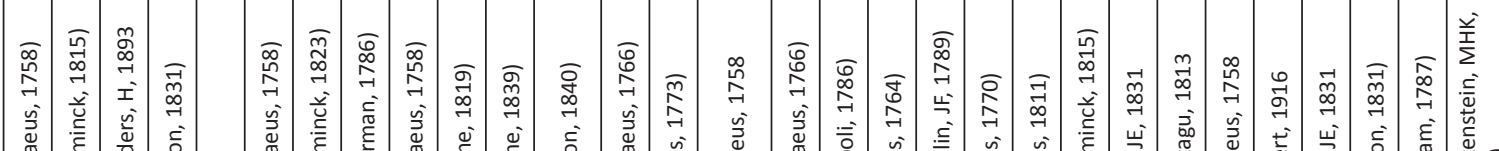

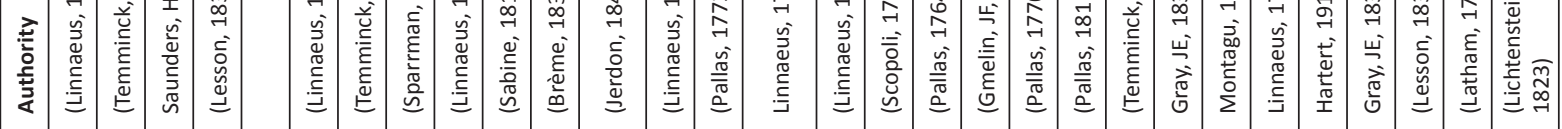

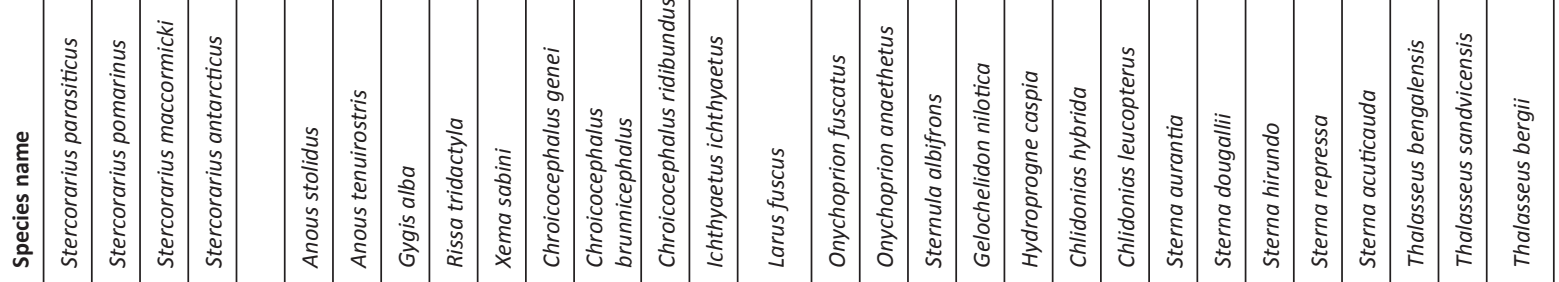

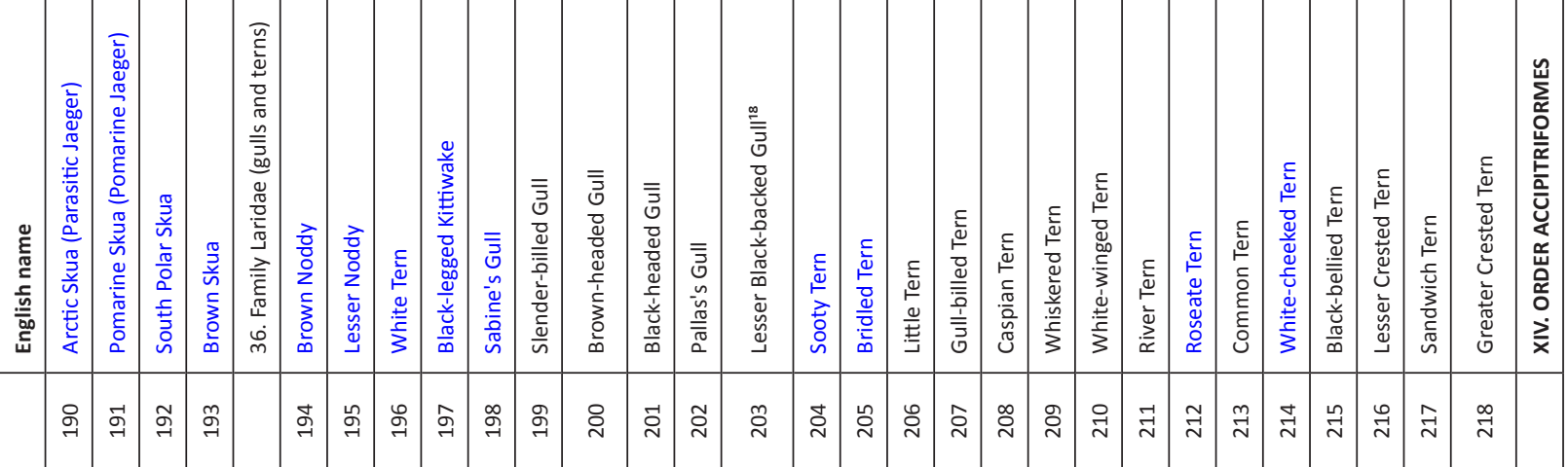




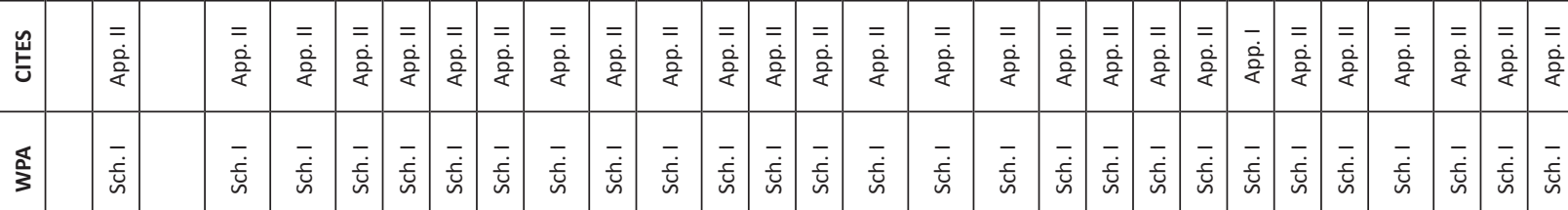

密

ว

\begin{tabular}{|c|c|c|c|c|c|c|c|c|c|c|c|c|c|c|c|c|c|c|c|c|c|c|c|}
\hline 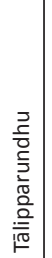 & 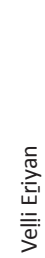 & 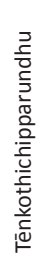 & 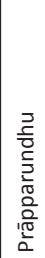 & 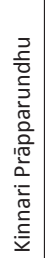 & 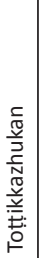 & 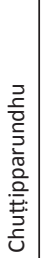 & 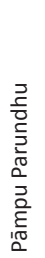 & 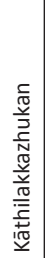 & 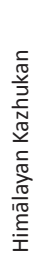 & 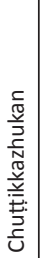 & 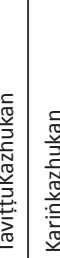 & 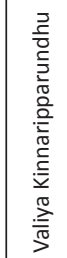 & 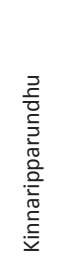 & 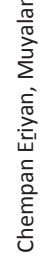 & 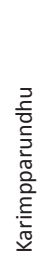 & 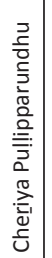 & 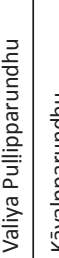 & 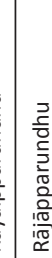 & 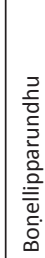 & 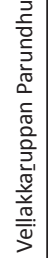 & 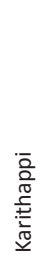 & 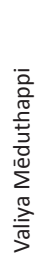 & 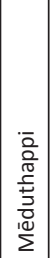 \\
\hline
\end{tabular}

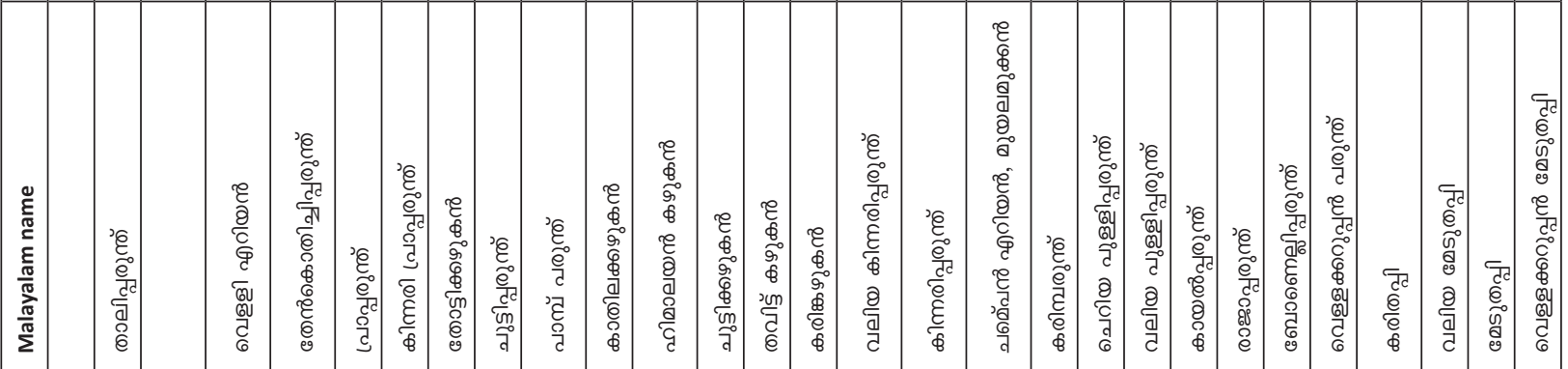

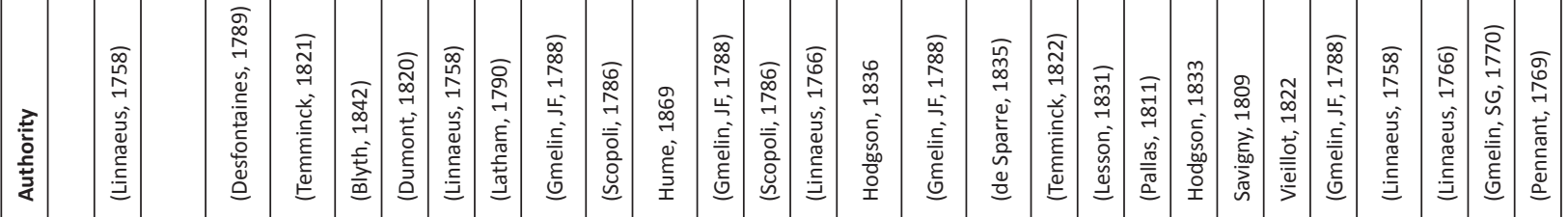

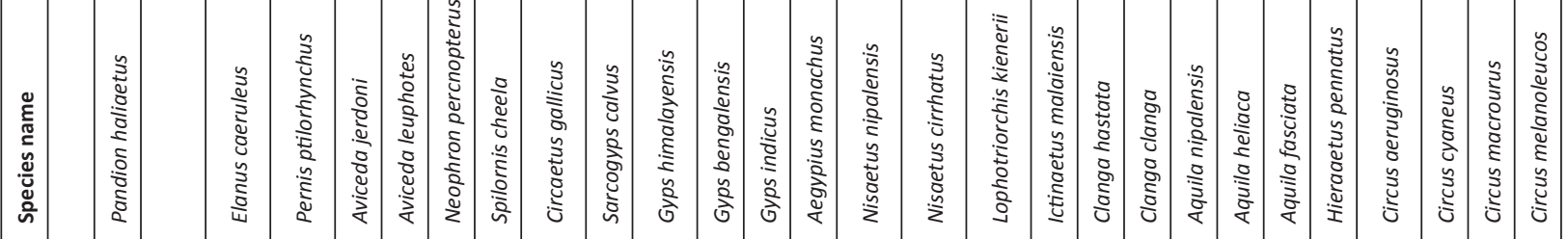

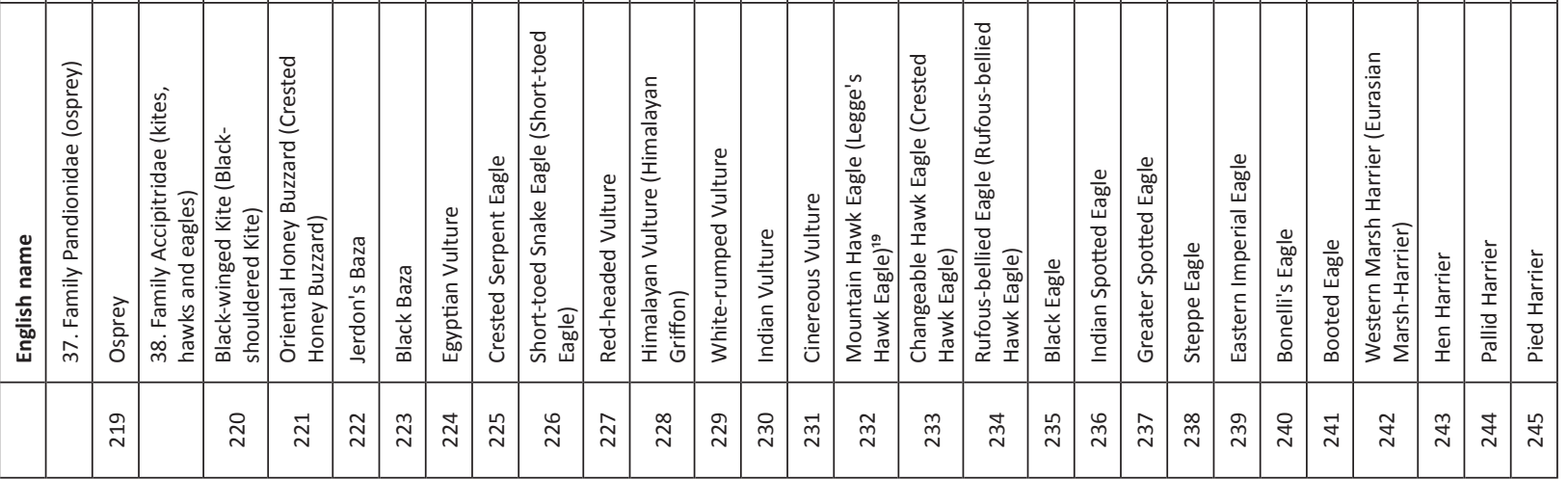




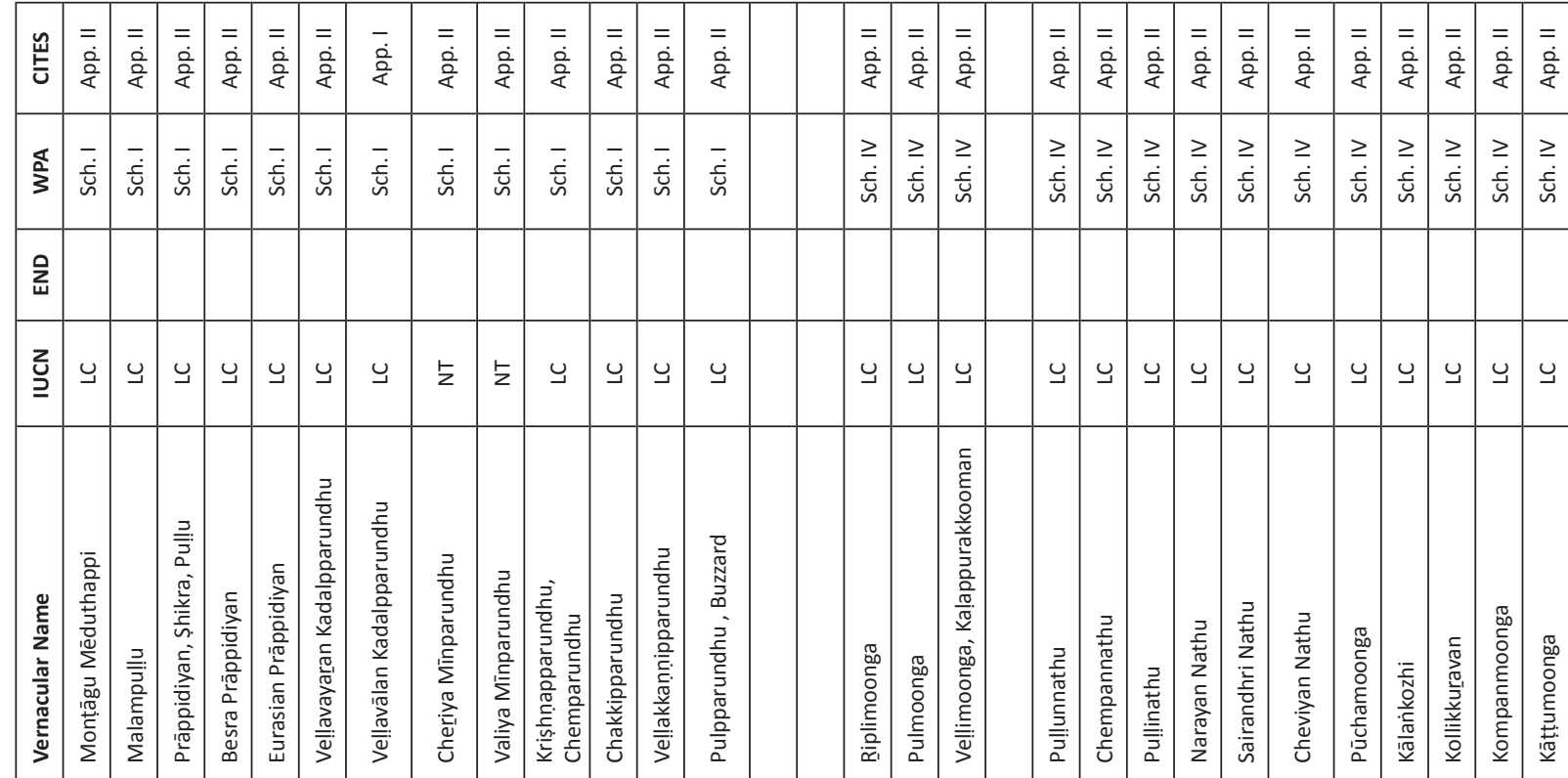

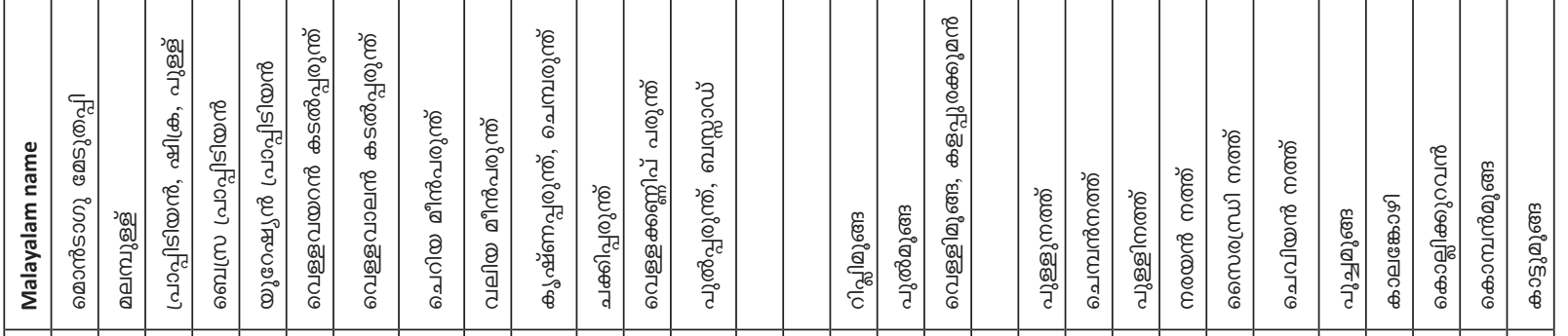

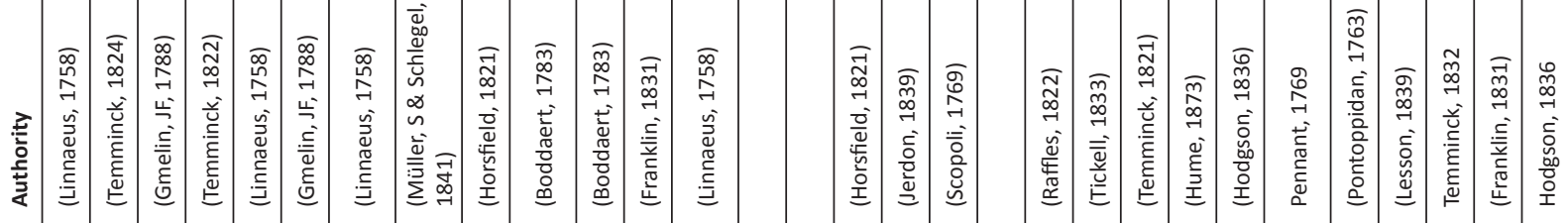

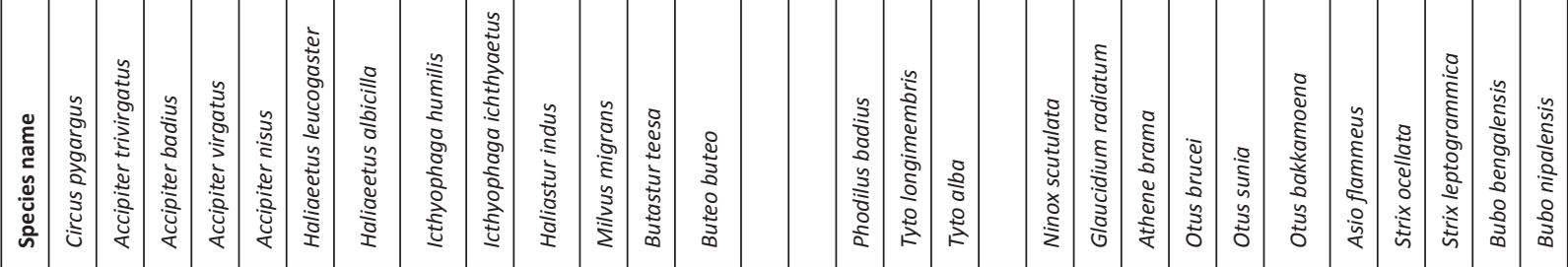




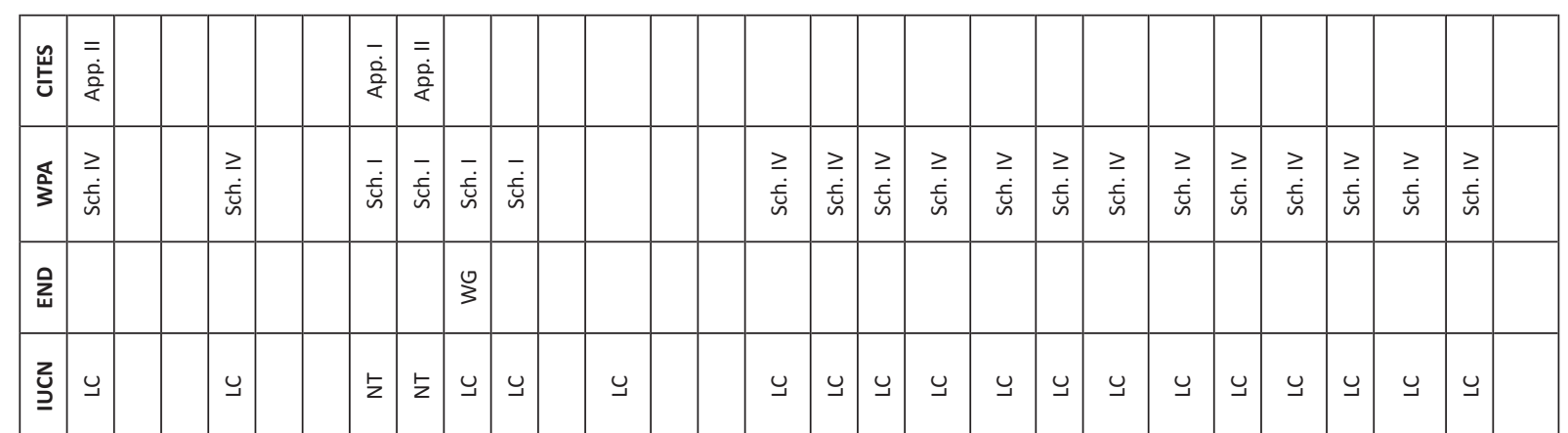

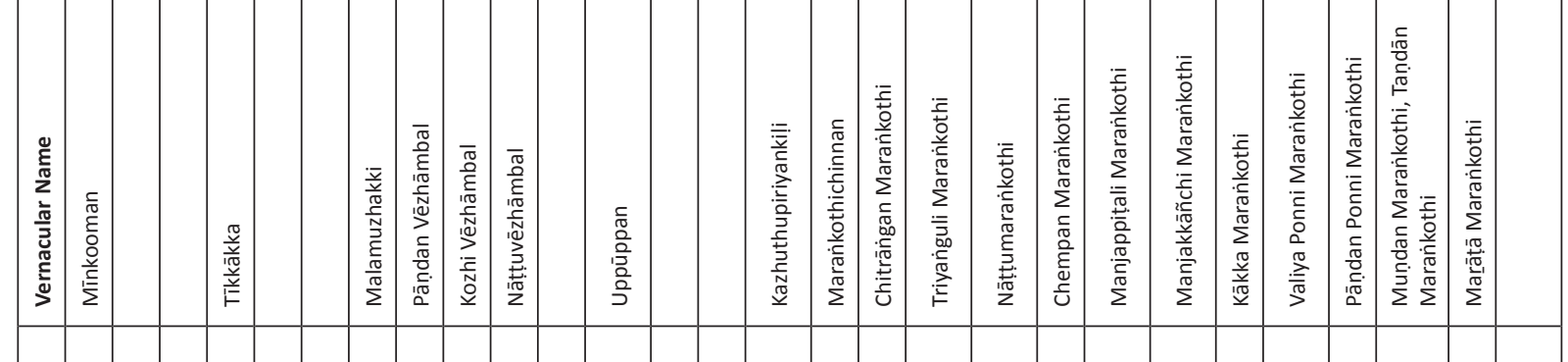

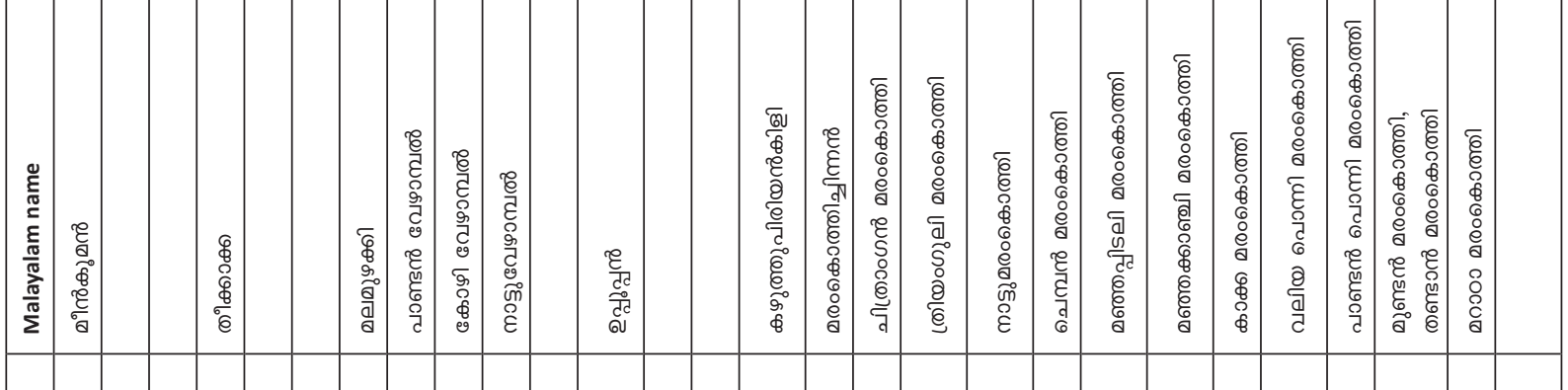

\begin{tabular}{|c|c|c|c|c|c|c|c|c|c|c|c|c|c|c|c|c|c|c|c|}
\hline & 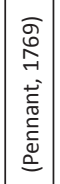 & 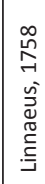 & 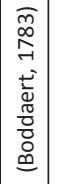 & 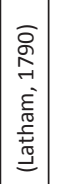 & 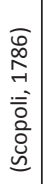 & 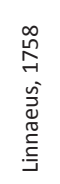 & 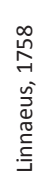 & 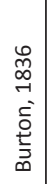 & 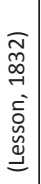 & 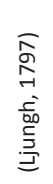 & 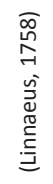 & 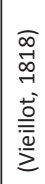 & 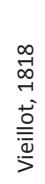 & 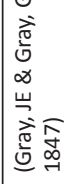 & 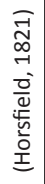 & 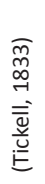 & 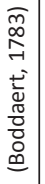 & 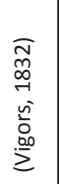 & 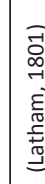 \\
\hline
\end{tabular}

\begin{tabular}{|c|c|c|c|c|c|c|c|c|c|c|c|c|c|c|c|c|c|c|c|c|c|c|}
\hline & 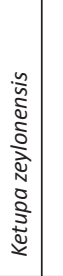 & & 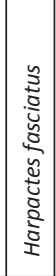 & & 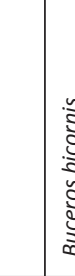 & 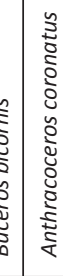 & 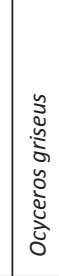 & 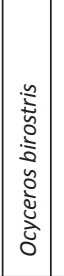 & & 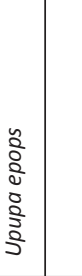 & & 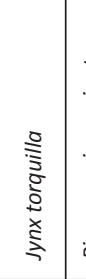 & 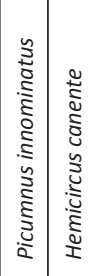 & 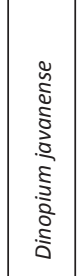 & 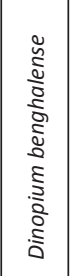 & 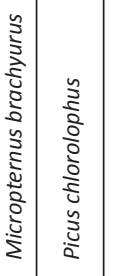 & 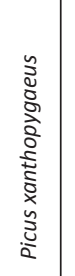 & 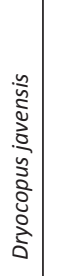 & 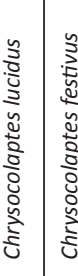 & 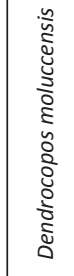 & 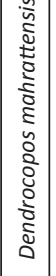 & \\
\hline 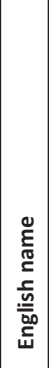 & 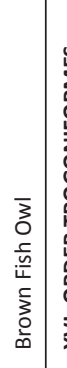 & 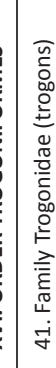 & 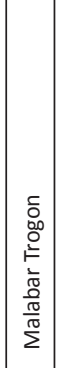 & 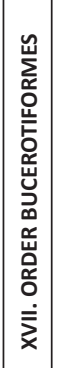 & 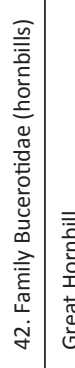 & 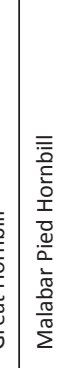 & 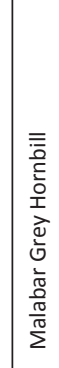 & 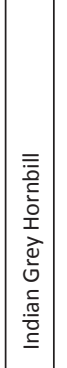 & 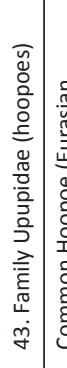 & 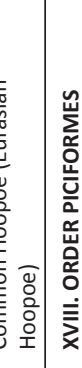 & 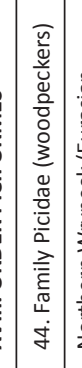 & 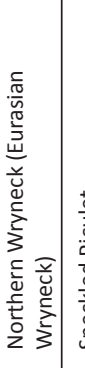 & 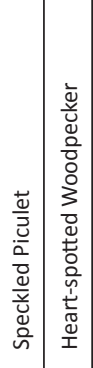 & 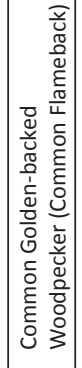 & 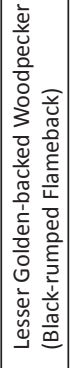 & 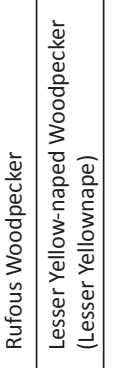 & 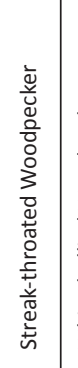 & 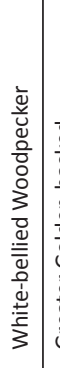 & 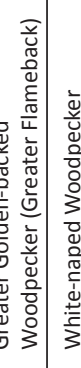 & 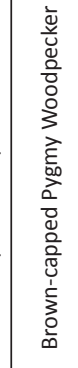 & 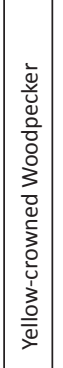 & 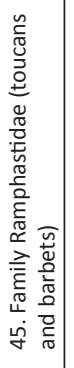 \\
\hline & $\stackrel{n}{ح}$ & & ? & & & $\widehat{\nwarrow}$ & i & $\stackrel{\infty}{\sim}$ & & $\stackrel{શ}{\approx}$ & & 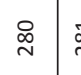 & \begin{tabular}{c|c}
$\stackrel{\vec{\infty}}{\sim}$ & $\widetilde{\infty}$ \\
$\sim$
\end{tabular} & 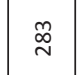 & $\stackrel{\Phi}{\sim}$ & 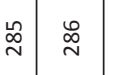 & $\stackrel{\infty}{\sim}$ & $\stackrel{\infty}{\stackrel{\infty}{\sim}}$ & $\stackrel{\mathscr{\infty}}{\sim}$ & వี & నี & \\
\hline
\end{tabular}




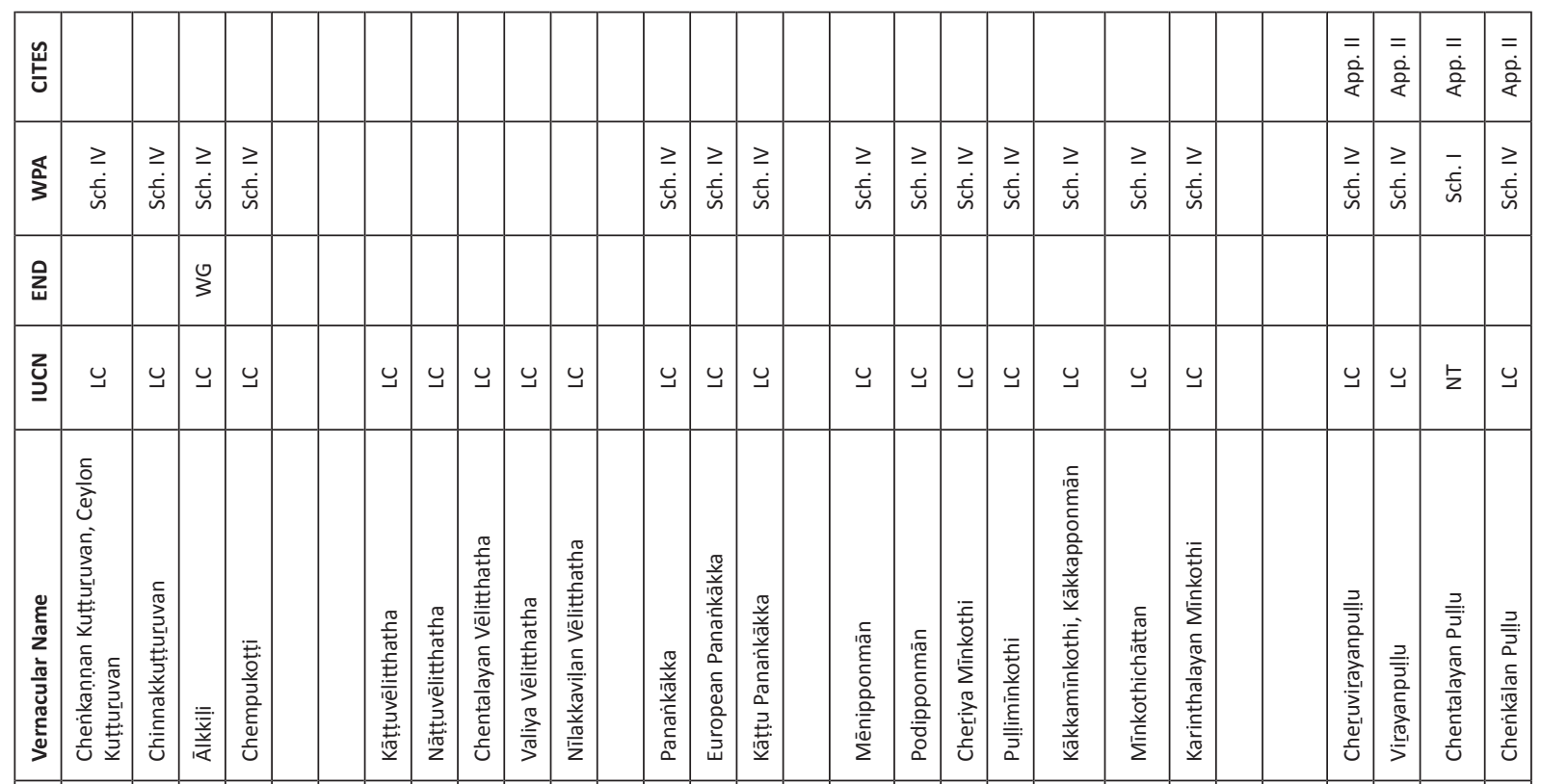

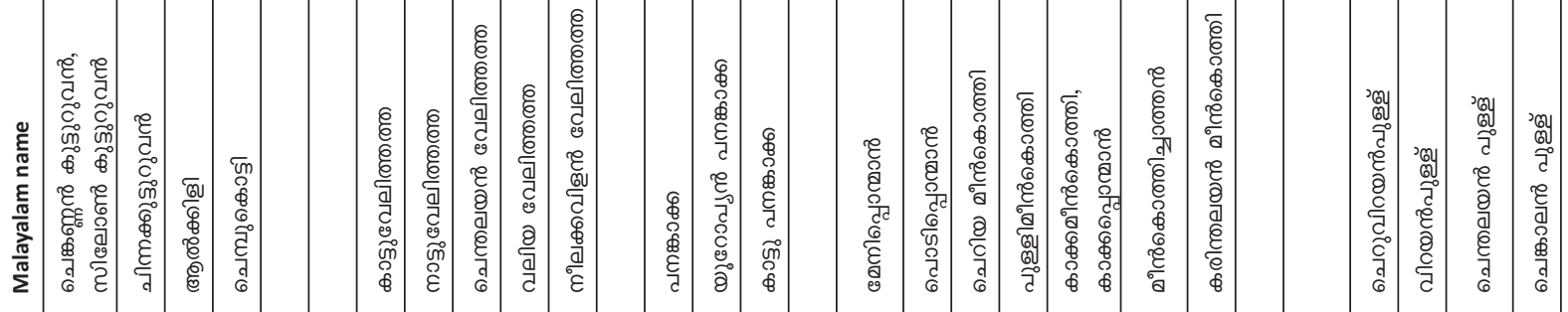

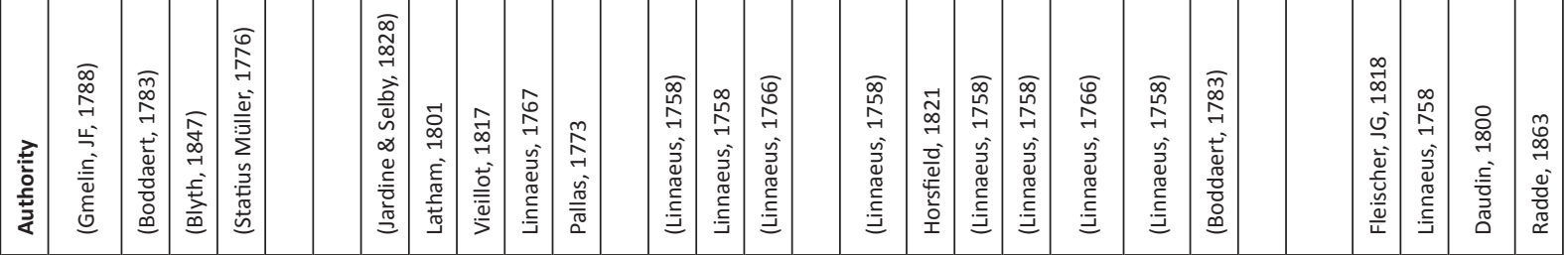

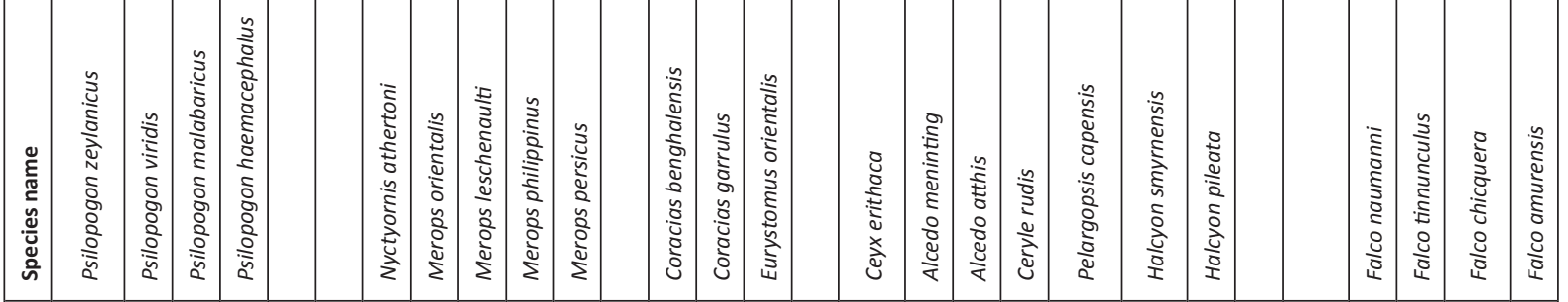

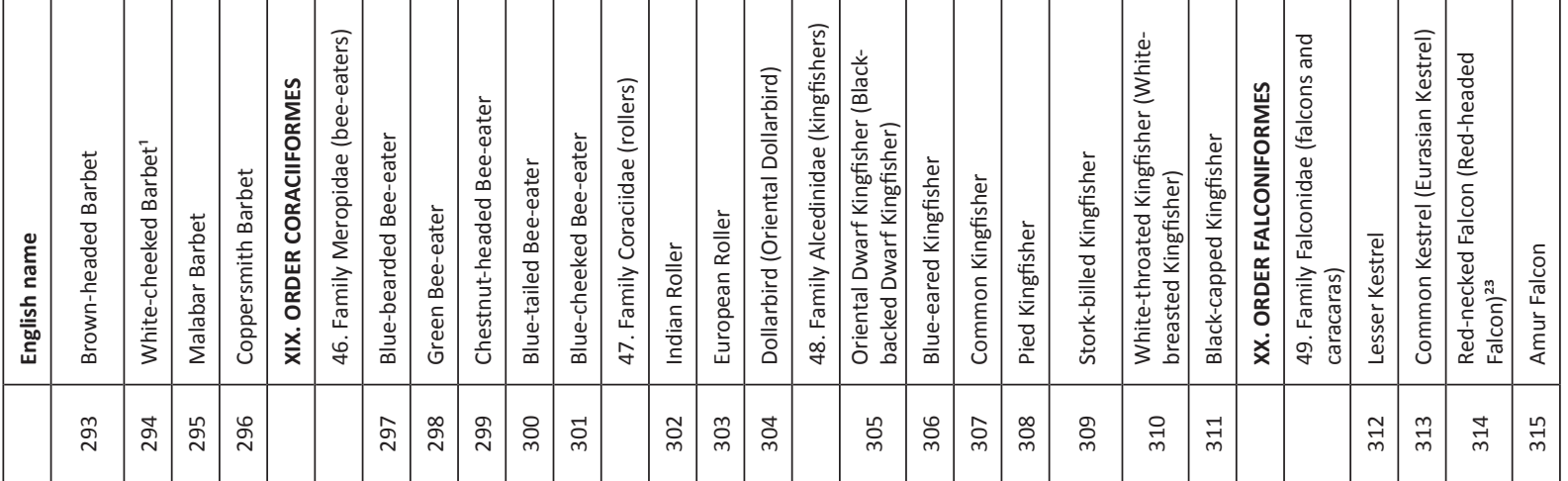




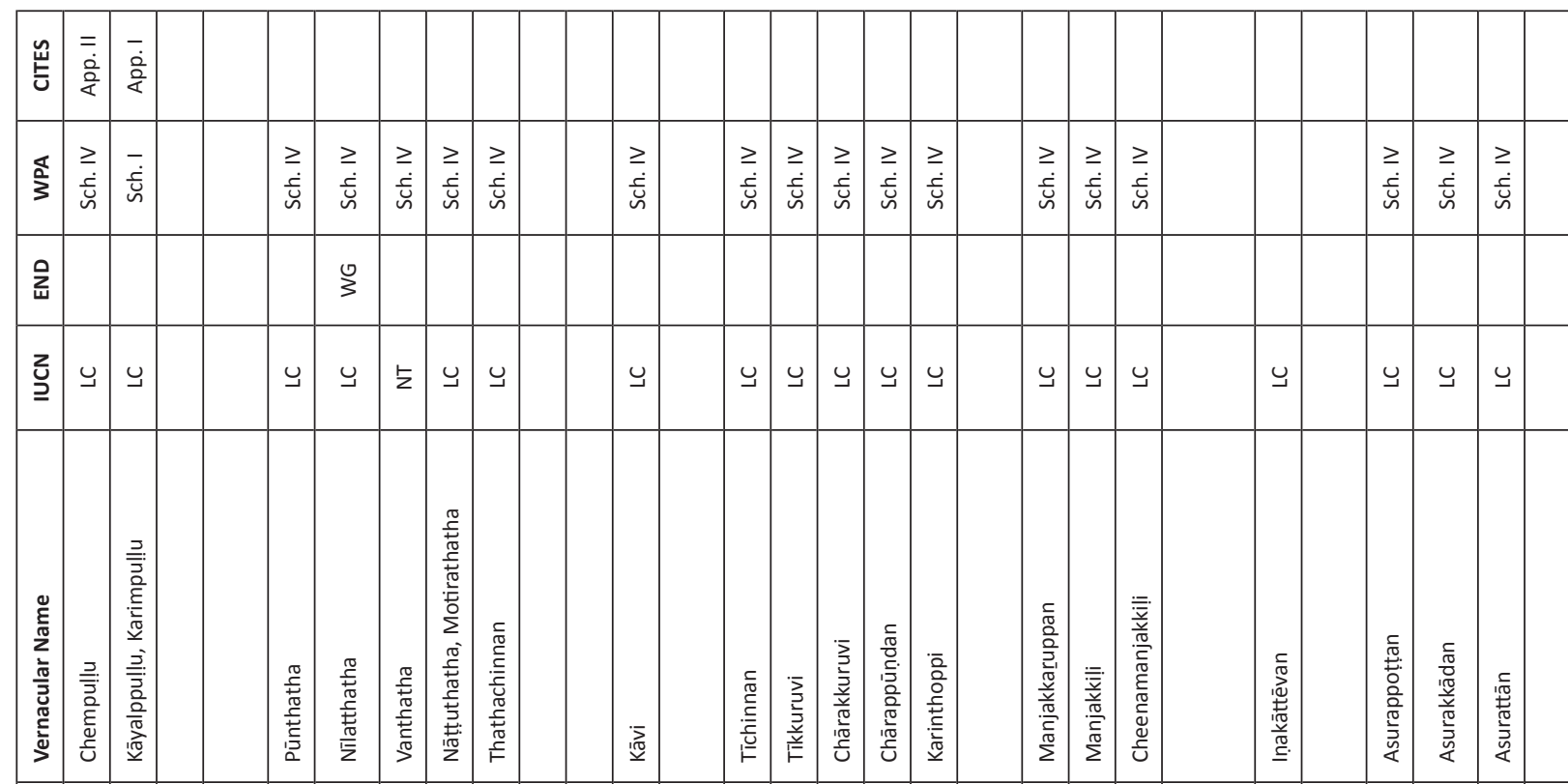

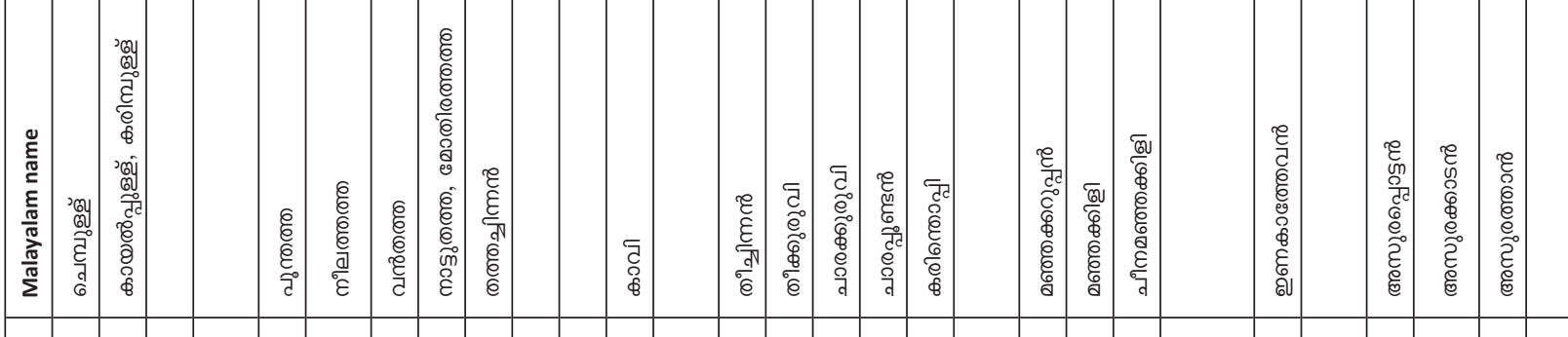

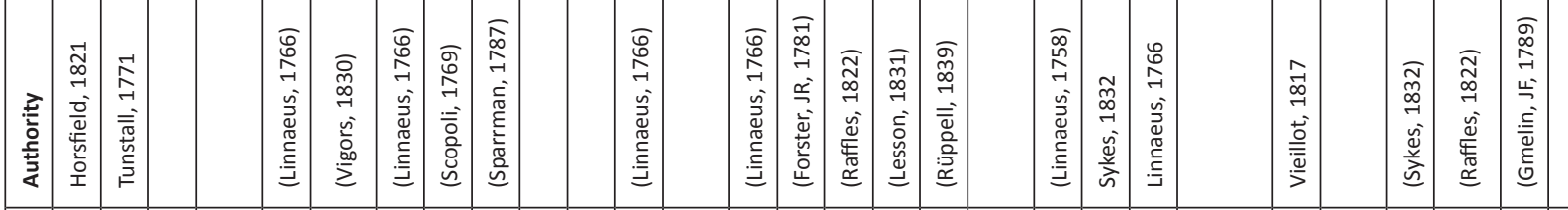

\begin{tabular}{|c|c|c|c|c|c|c|c|c|c|c|c|c|c|c|c|c|c|c|c|c|c|c|}
\hline & 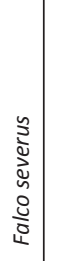 & 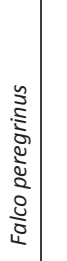 & & 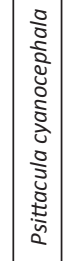 & 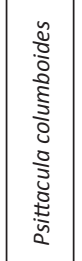 & 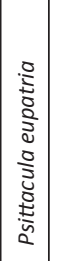 & 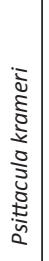 & 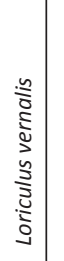 & & 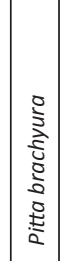 & & 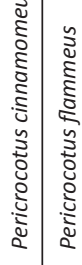 & 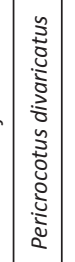 & 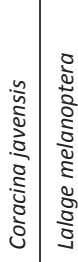 & & 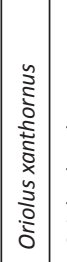 & 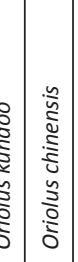 & & 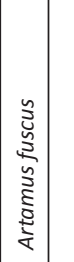 & & 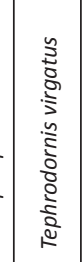 & 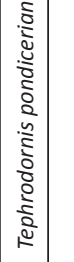 \\
\hline 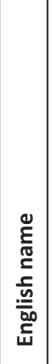 & 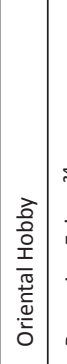 & 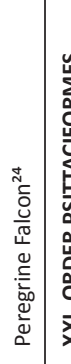 & 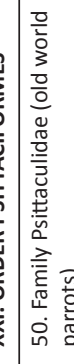 & 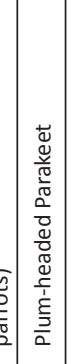 & 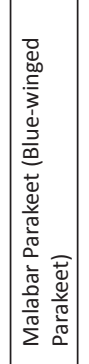 & 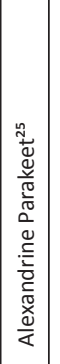 & 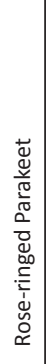 & 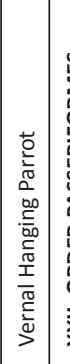 & 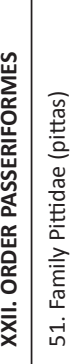 & 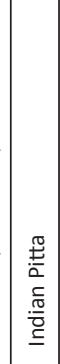 & 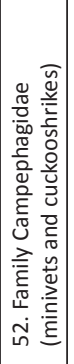 & 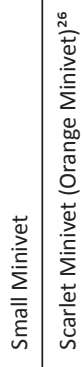 & 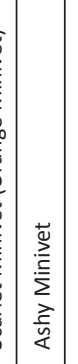 & 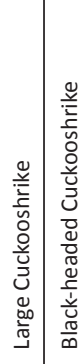 & 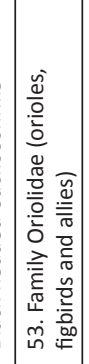 & 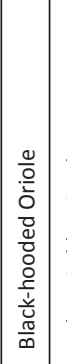 & 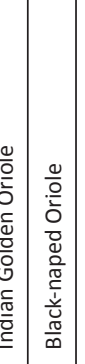 & 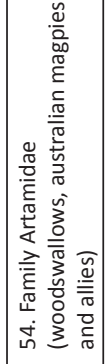 & 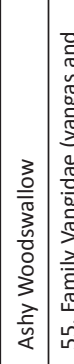 & 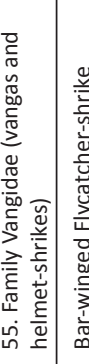 & 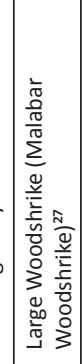 & 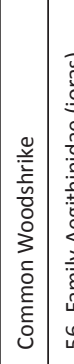 \\
\hline & 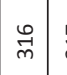 & $\vec{m}$ & & $\stackrel{\infty}{m}$ & $\stackrel{\vec{m}}{\vec{m}}$ & ᄅ్ & $\overrightarrow{\widetilde{m}}$ & $\underset{\tilde{m}}{\mathbb{Z}}$ & & $\underset{\sim}{\tilde{m}}$ & & $\underset{\sim}{\mathbb{N}} \mid \stackrel{\sim}{\sim}$ & $\underset{\sim}{\stackrel{\infty}{m}}$ & 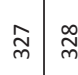 & & & $\begin{array}{l}\text { Di } \\
\vec{m}\end{array}$ & & $\tilde{m}$ & $m$ & 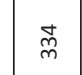 & 恖 \\
\hline
\end{tabular}




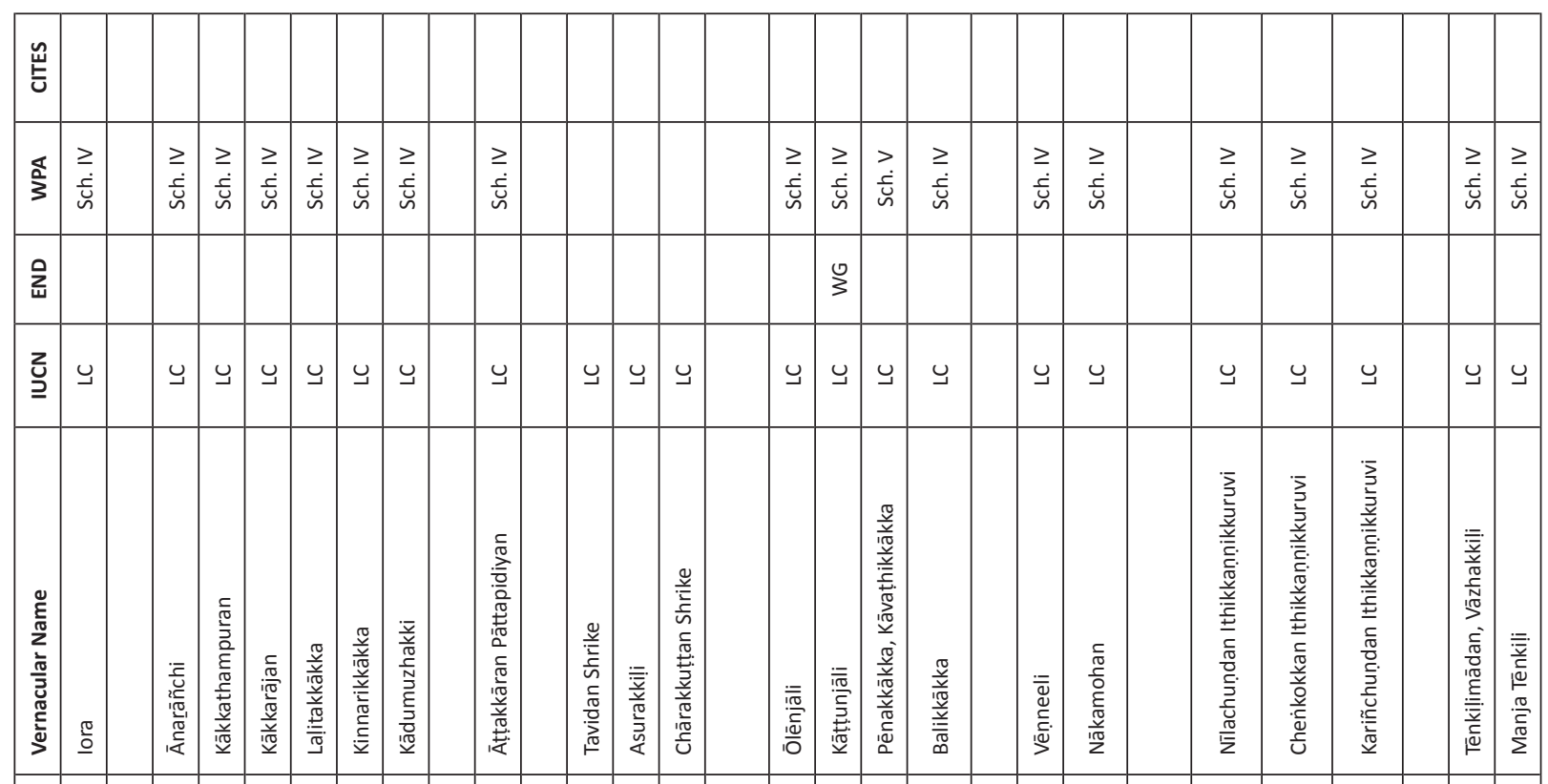

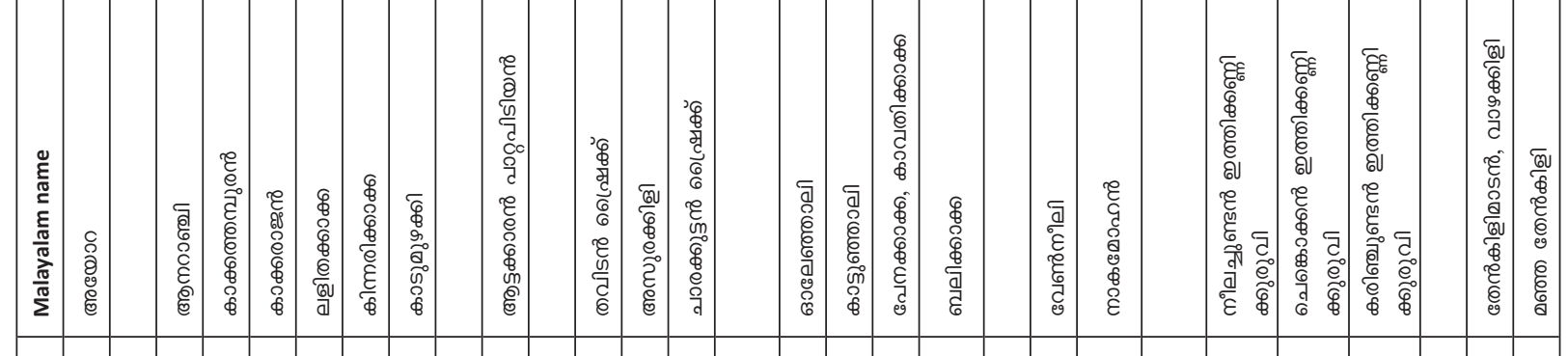

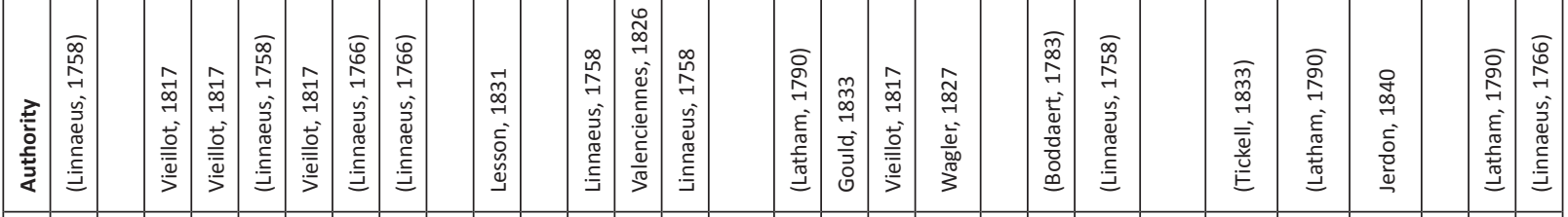

\begin{tabular}{|c|c|c|c|c|c|c|c|c|c|c|c|c|c|c|c|c|c|c|}
\hline 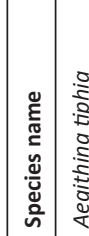 & 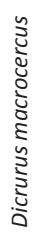 & & 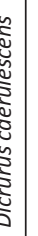 & 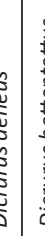 & 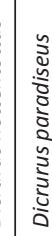 & 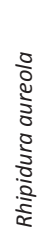 & 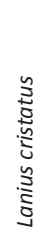 & 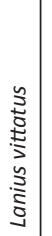 & 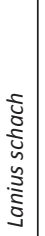 & 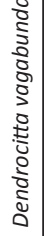 & 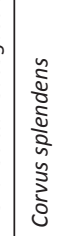 & 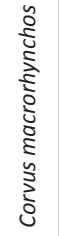 & 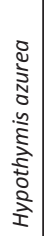 & 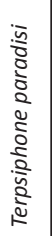 & 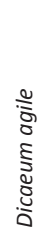 & 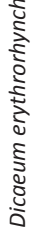 & 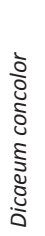 & 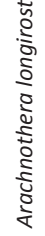 \\
\hline
\end{tabular}

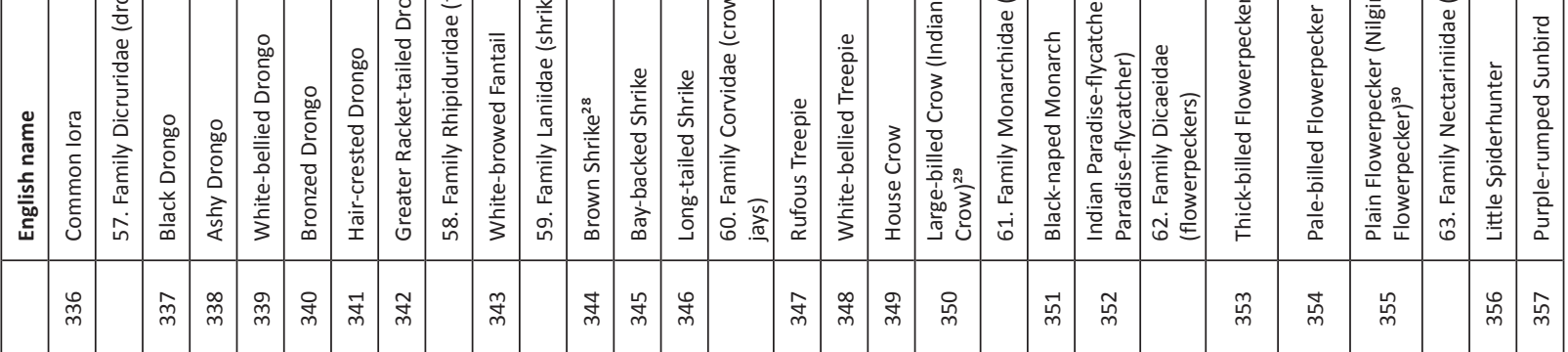




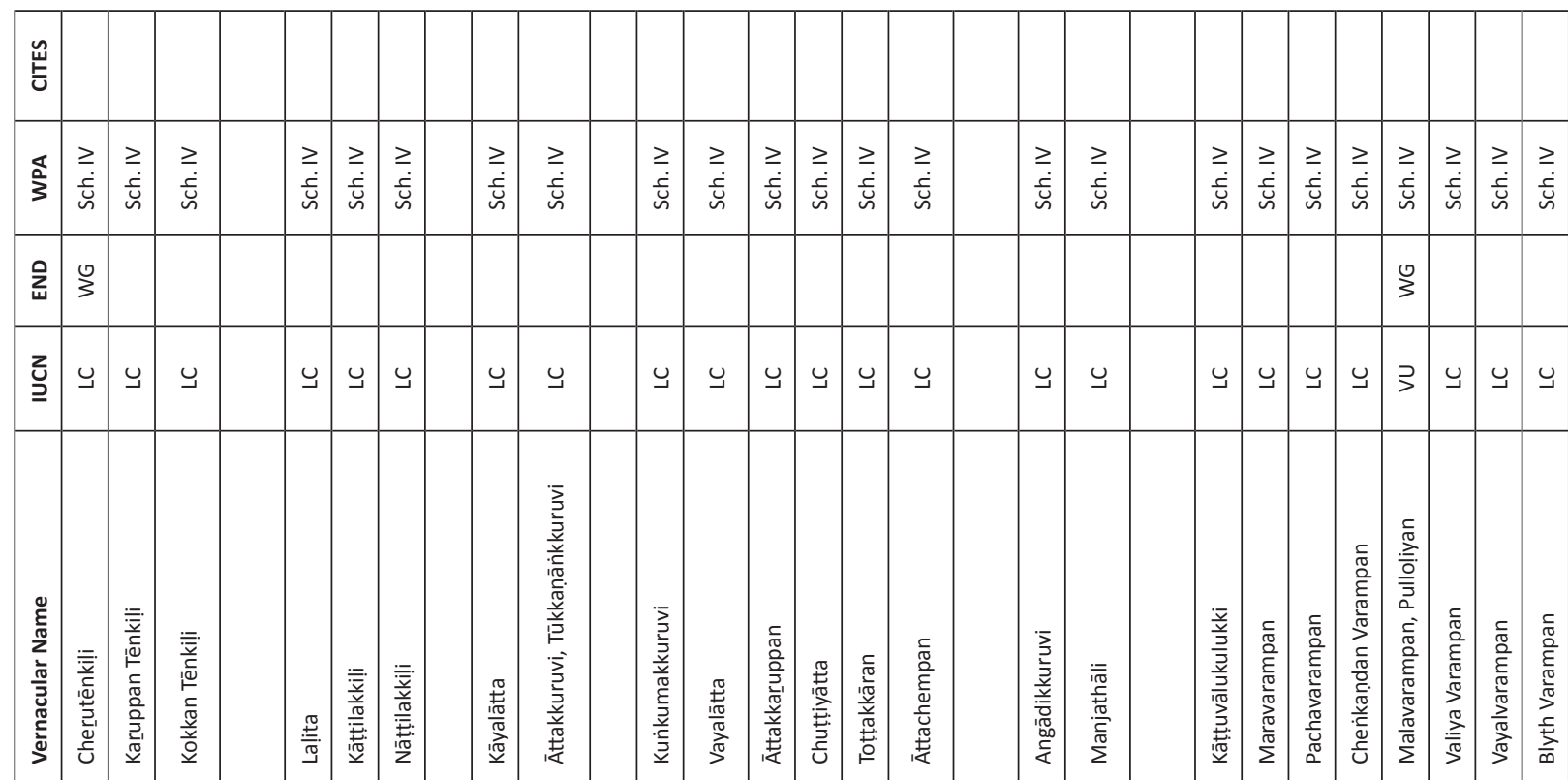

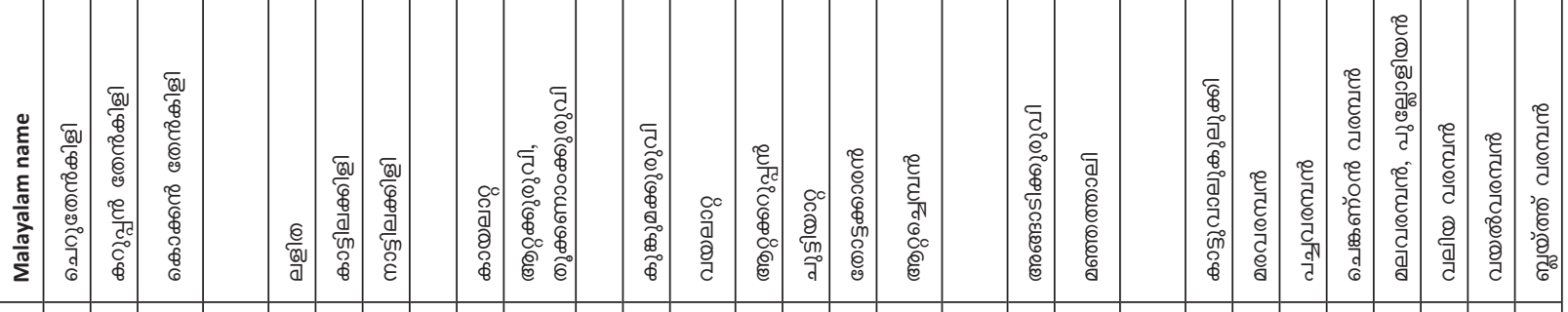

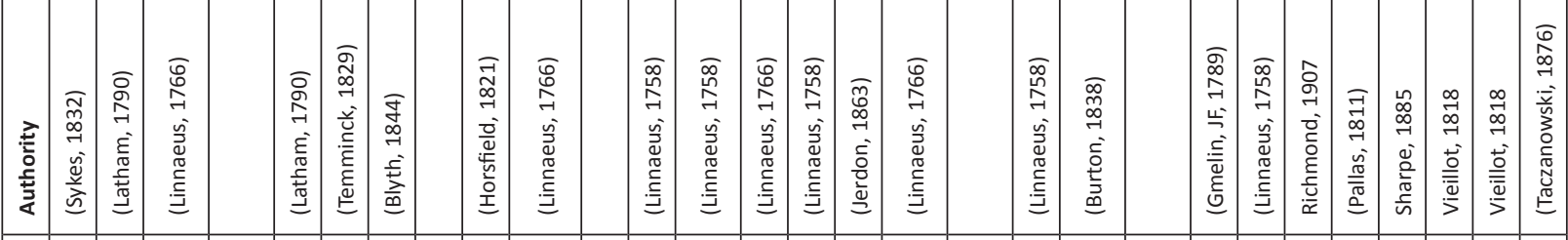

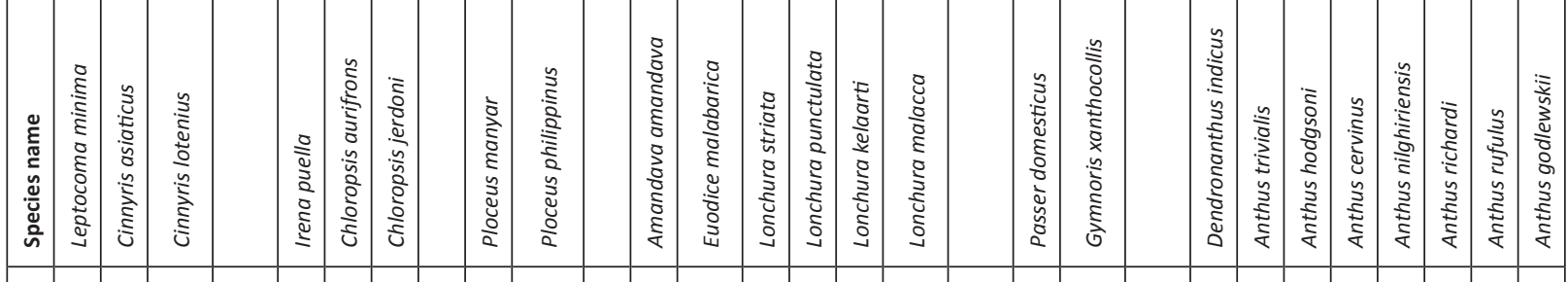

\begin{tabular}{|c|c|c|c|c|c|c|c|c|c|c|c|c|c|c|c|c|c|c|c|c|c|c|c|c|c|c|}
\hline 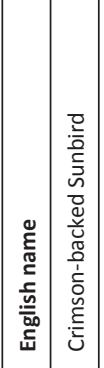 & 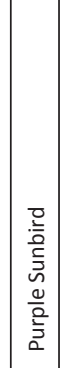 & 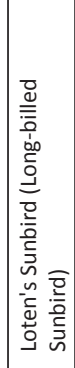 & 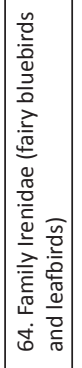 & 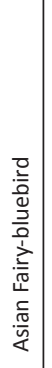 & 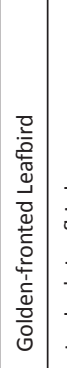 & 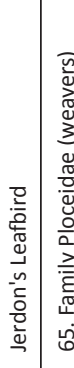 & 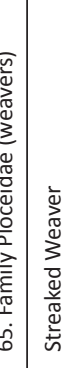 & 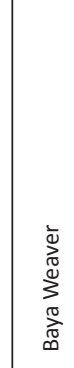 & 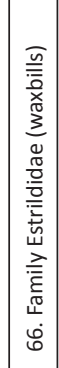 & 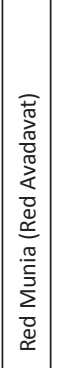 & 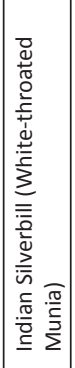 & 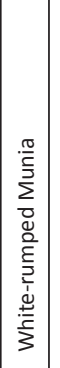 & 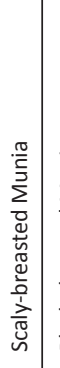 & 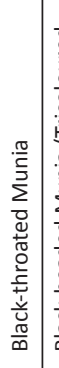 & 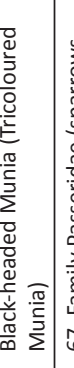 & 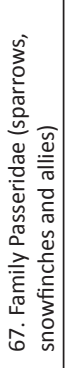 & 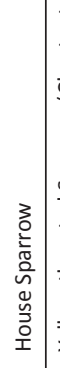 & 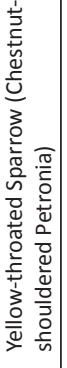 & 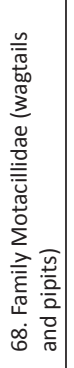 & 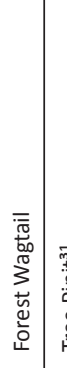 & 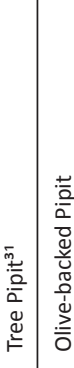 & 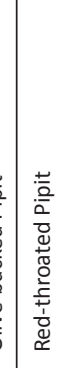 & 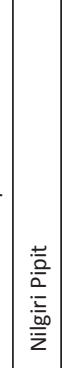 & 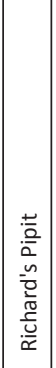 & 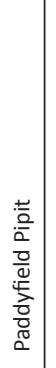 & 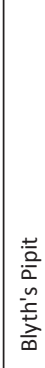 \\
\hline 怘 & 品 & $\underset{m}{\stackrel{\circ}{0}}$ & & $\overrightarrow{⿱ 0}$ & : & 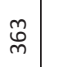 & :్ల & 怘 & & 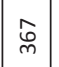 & $\stackrel{\infty}{\stackrel{\infty}{m}}$ & 怘 & : & $\stackrel{?}{m}$ & 命 & & $\underset{m}{N}$ & $\stackrel{m}{m}$ & & $\underset{m}{D}$ & $\stackrel{n}{m}$ & 命 & $\stackrel{\infty}{m}$ & $\stackrel{a}{m}$ & $\underset{m}{\infty}$ & mo \\
\hline
\end{tabular}




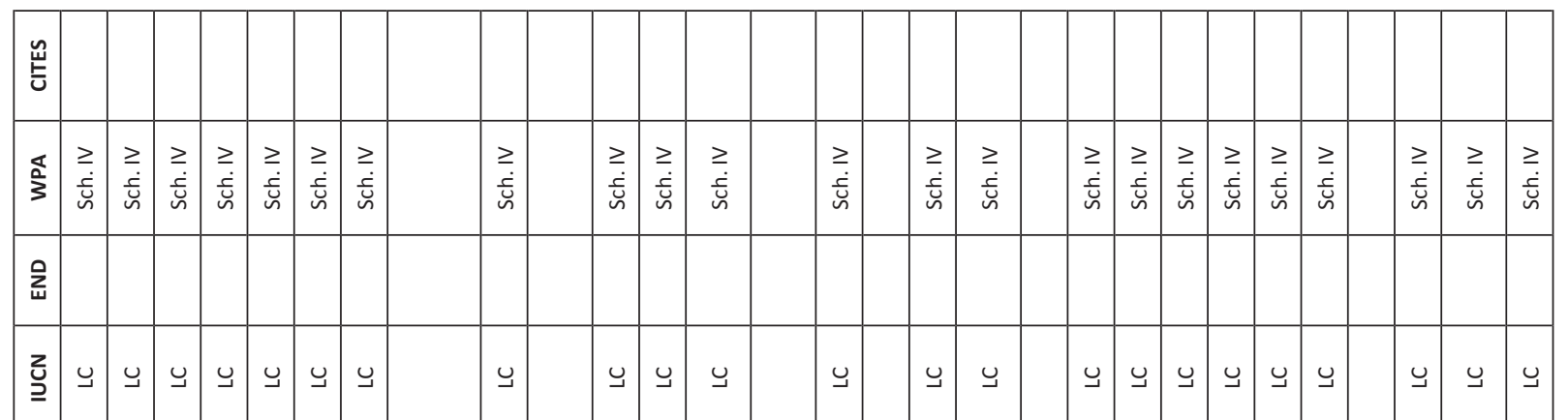

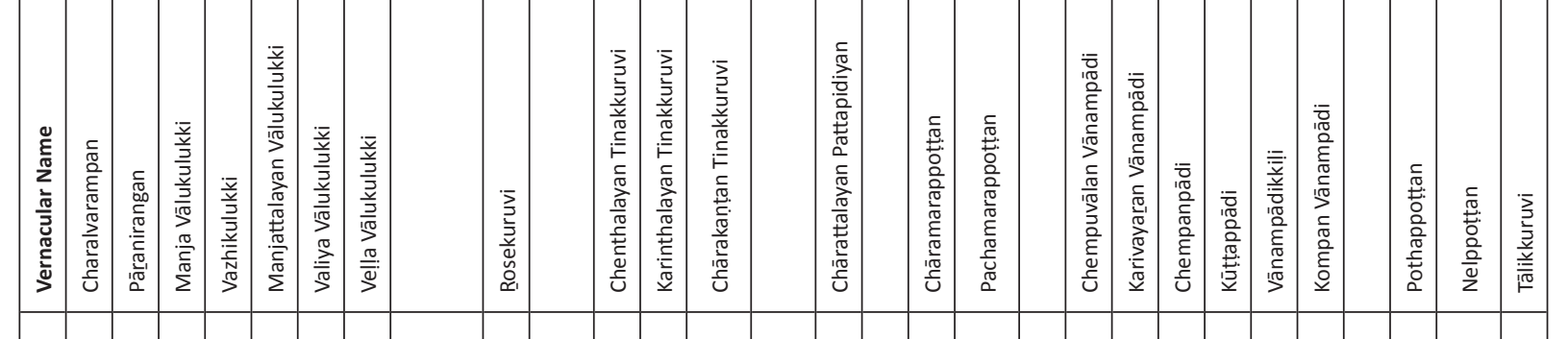

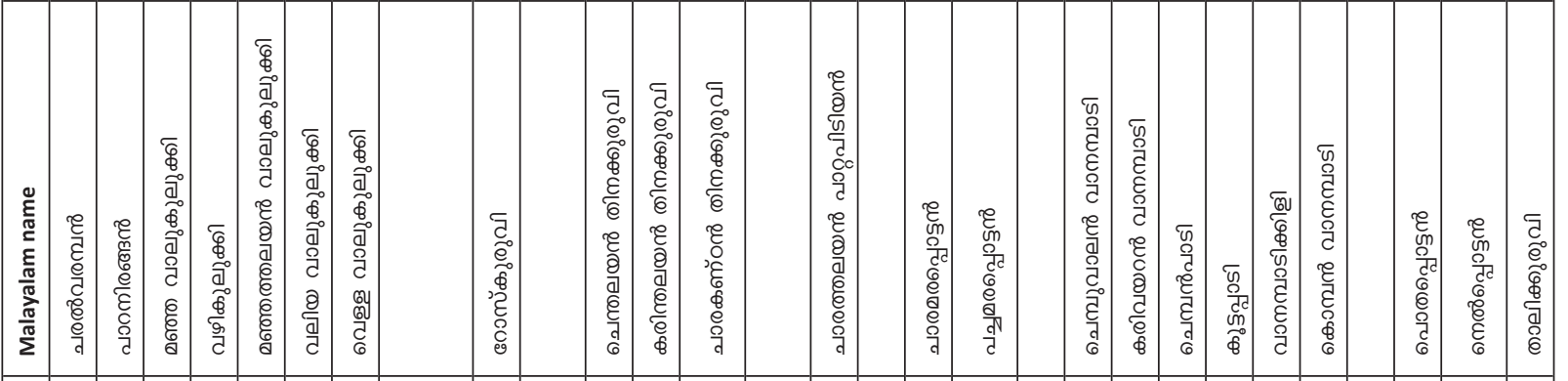

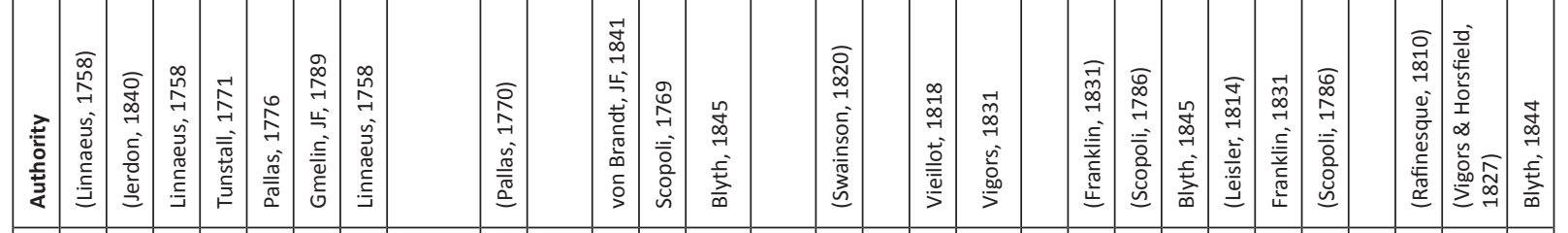

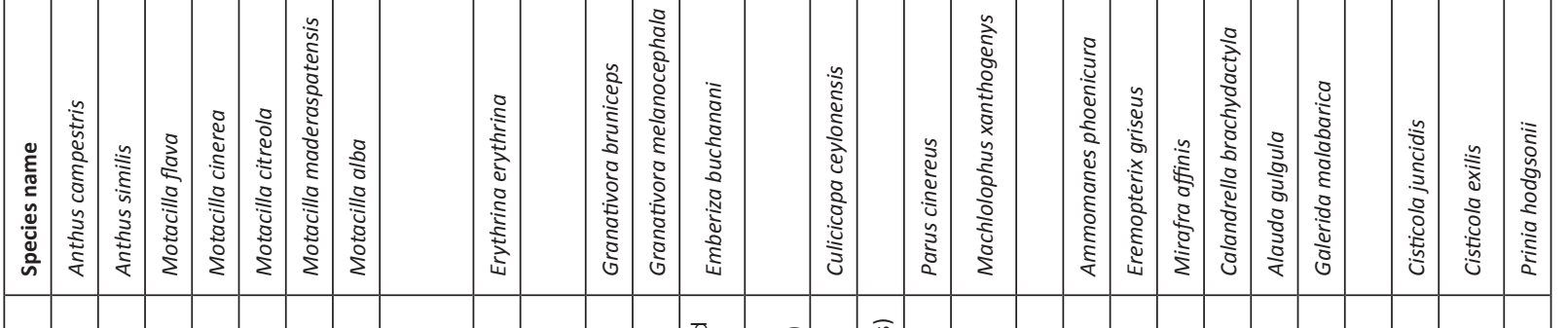

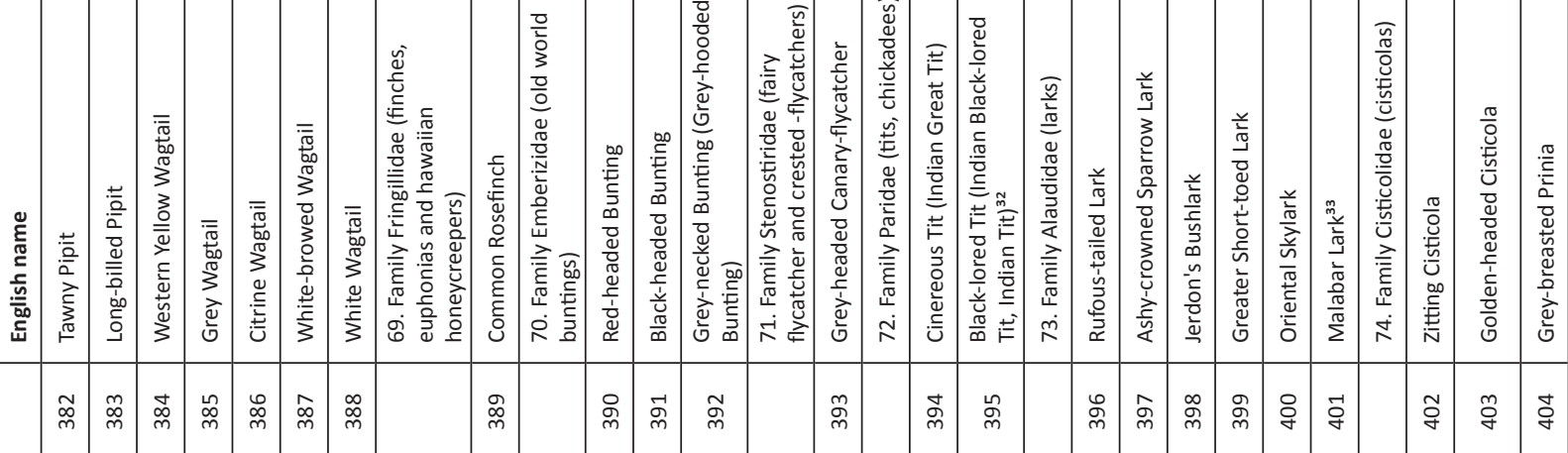




\begin{tabular}{|l|l|l|l|l|l|l|l|l|l|l|l|l|l|l|l|l|l|l|l|l|l|l|l|l|l|}
\hline$\underline{u}$ & & & & & & & & & & & & & & & & & & & & & & & & & \\
\hline
\end{tabular}

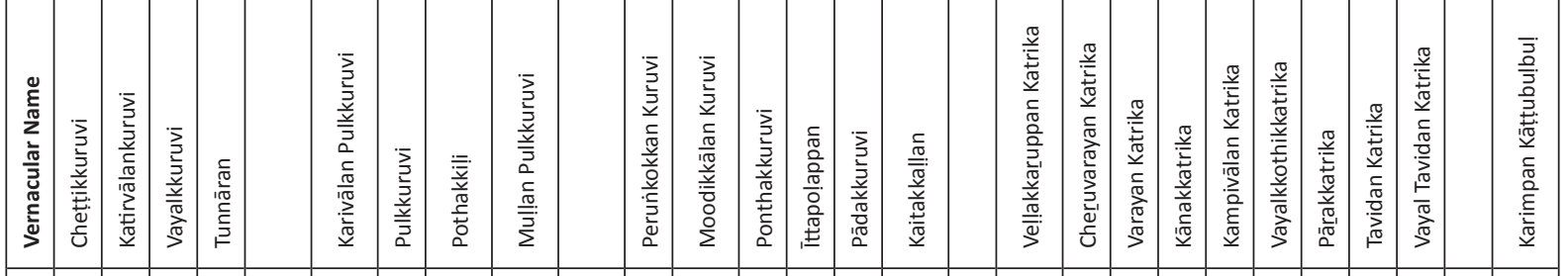

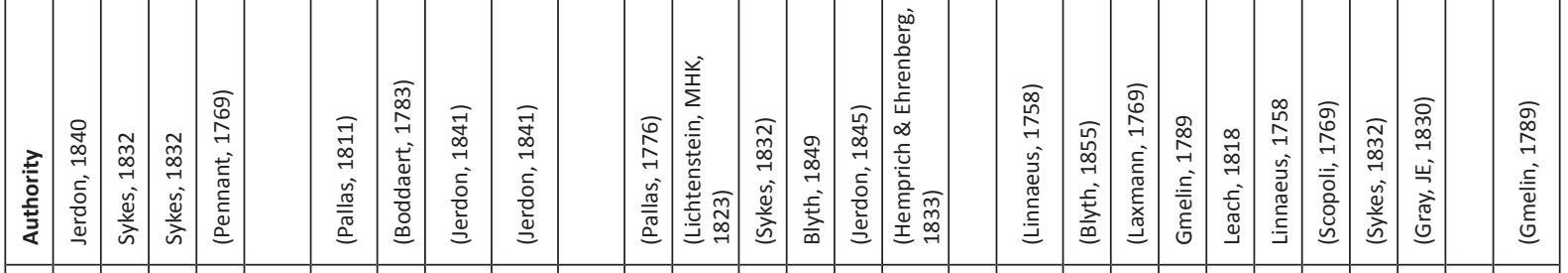

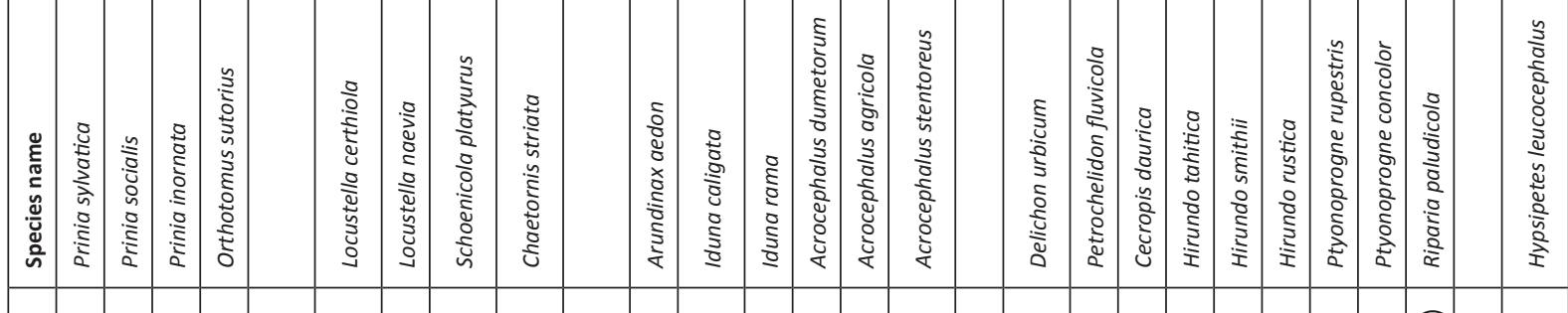

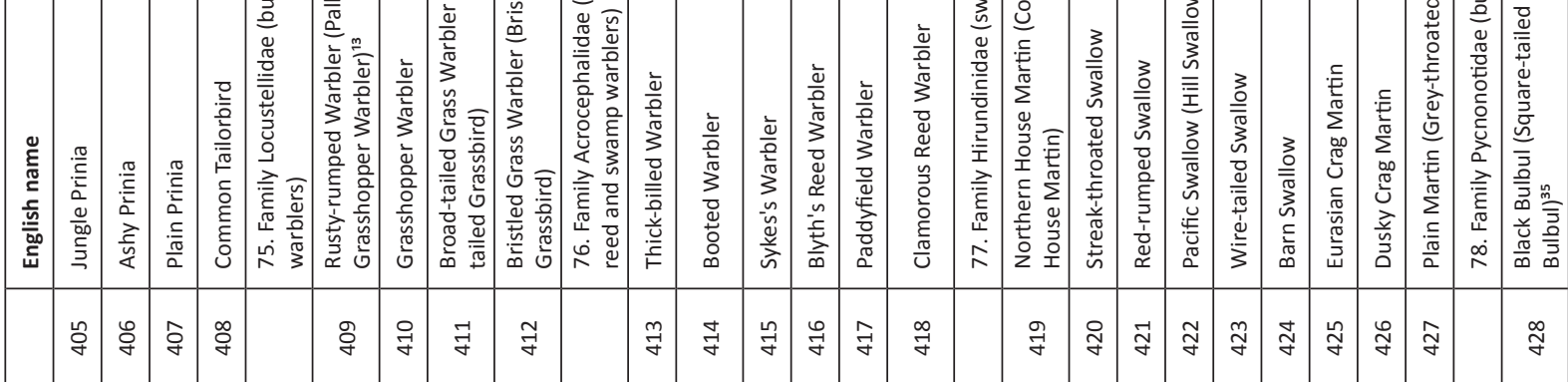




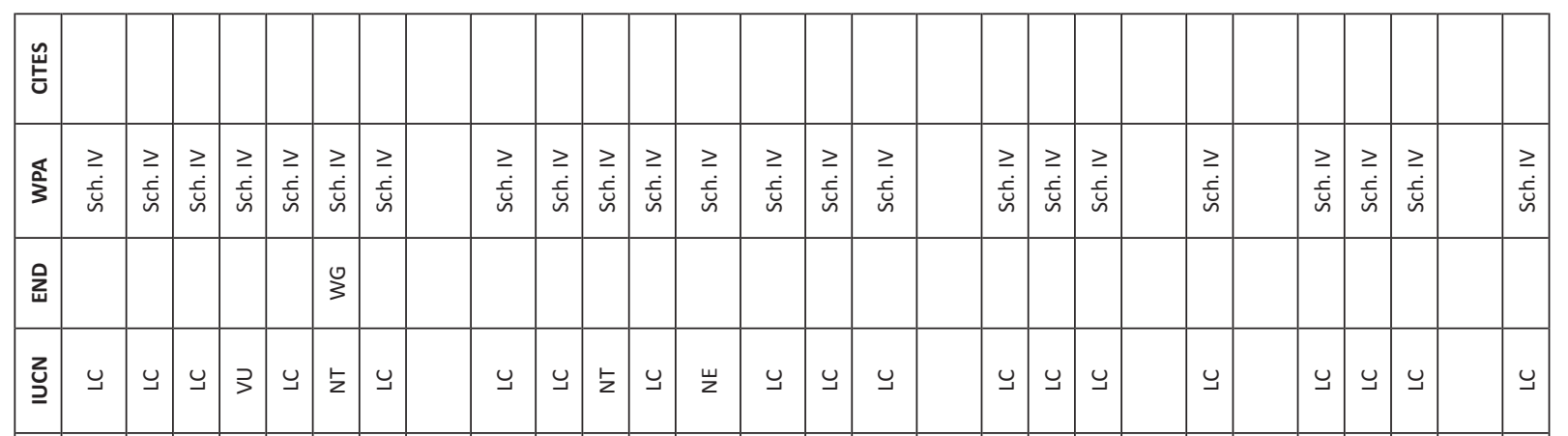

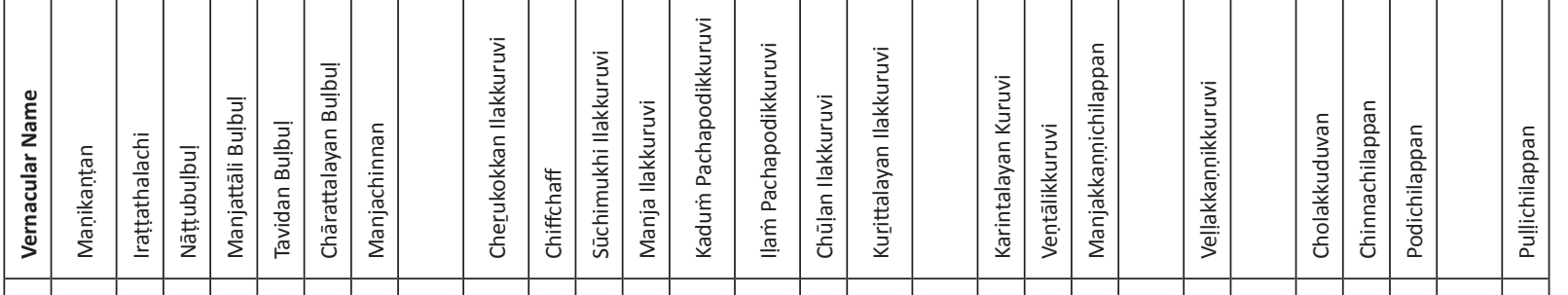

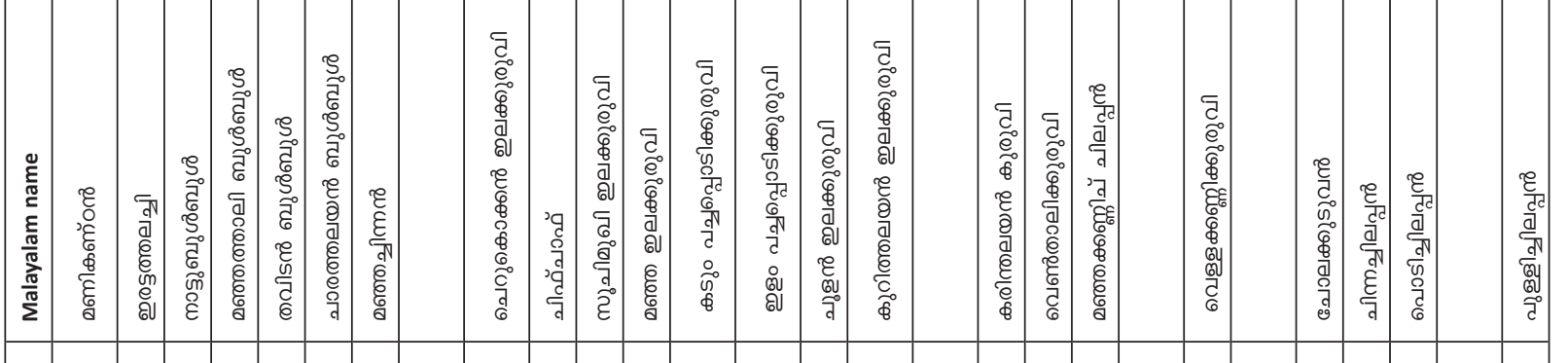

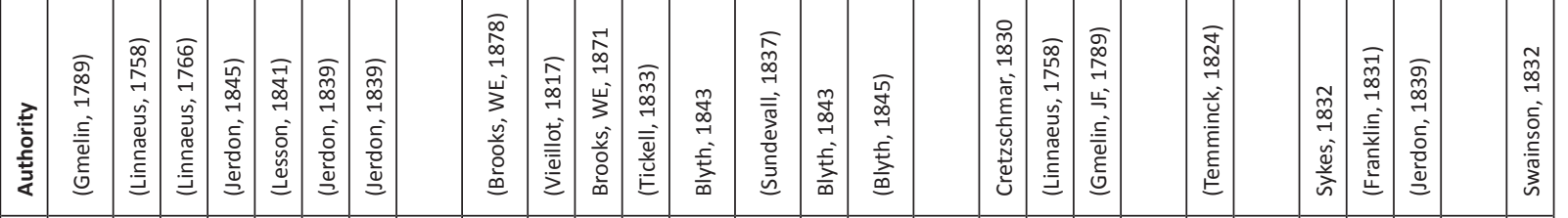

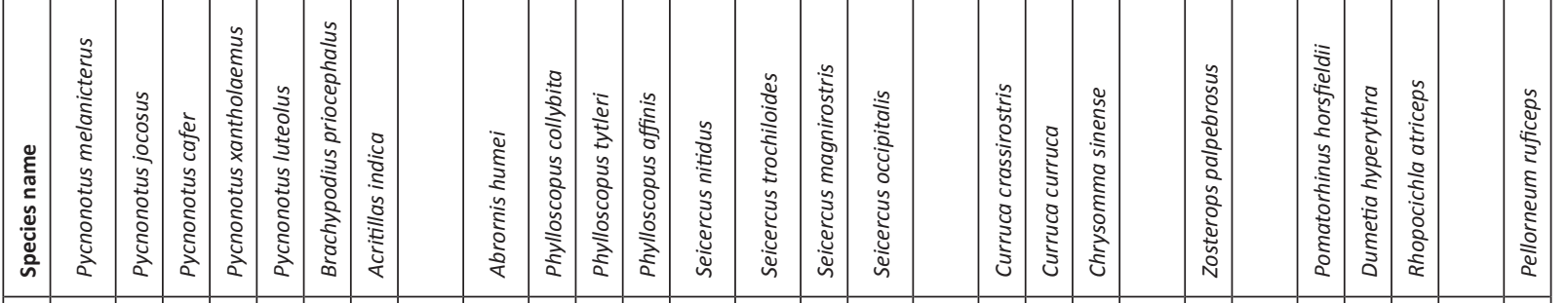

\begin{tabular}{|c|c|c|c|c|c|c|c|c|c|c|c|c|c|c|c|c|c|c|c|c|c|c|}
\hline 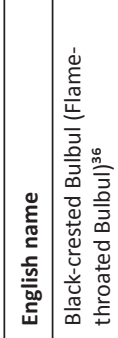 & 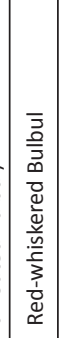 & 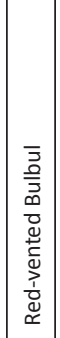 & 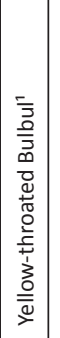 & 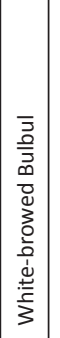 & 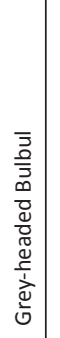 & 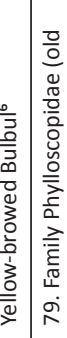 & 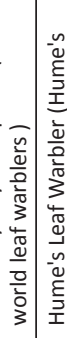 & 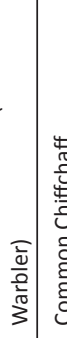 & 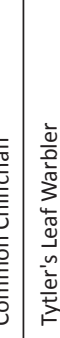 & 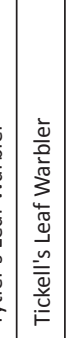 & 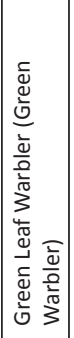 & 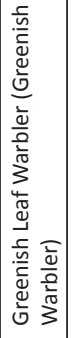 & 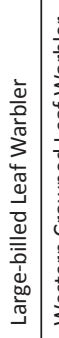 & 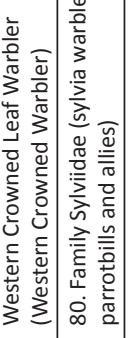 & 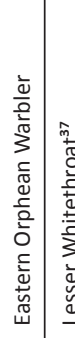 & 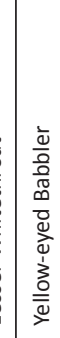 & 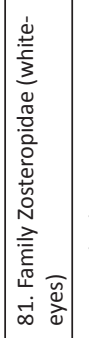 & 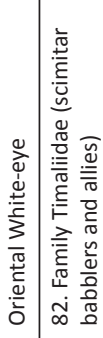 & 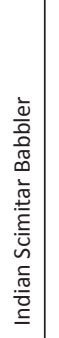 & 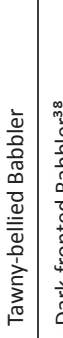 & 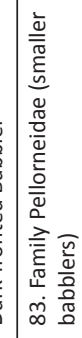 & 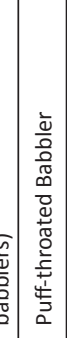 \\
\hline જి & $\stackrel{\circ}{q}$ & $\vec{\xi}$ & $\tilde{\tilde{q}}$ & $\stackrel{m}{y}$ & 奨 & 岁 & 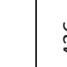 & 曾 & 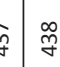 & 学 & g & $\vec{F}$ & ₹ & g & \begin{tabular}{l|l} 
\\
\end{tabular} & 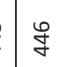 & & f & $\stackrel{\infty}{q}$ & $g$ & & 学 \\
\hline
\end{tabular}




\begin{tabular}{|c|c|c|c|c|c|c|c|c|c|c|c|c|c|c|c|c|c|c|c|c|c|c|c|}
\hline 峞 & & & & & & & & & & & & & & & & & $\begin{array}{l}\overline{\overline{3}} \\
\overline{\frac{0}{4}}\end{array}$ & & & & & & \\
\hline$\frac{\widehat{a}}{3}$ & $\begin{array}{l}\geq \\
\text { i } \\
\text { n. }\end{array}$ & $\begin{array}{l}\geq \\
\geq \\
\dot{\underline{\omega}}\end{array}$ & $\begin{array}{l}\geq \\
\geq \\
\dot{\omega} \\
\end{array}$ & $\begin{array}{l}\geq \\
\dot{i} \\
\dot{\omega}\end{array}$ & 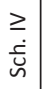 & $\begin{array}{l}\geq \\
\geq \\
\text { 忘 }\end{array}$ & $\begin{array}{l}\geq \\
\text { i } \\
\dot{\omega}\end{array}$ & $\begin{array}{l}\geq \\
\text { i } \\
\text { in }\end{array}$ & & & $\begin{array}{l}\geq \\
\text { i } \\
\text { i }\end{array}$ & 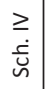 & $\begin{array}{l}\geq \\
\stackrel{\vec{i}}{\dot{n}}\end{array}$ & 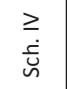 & $\begin{array}{l}\geq \\
\stackrel{\vec{i}}{\dot{n}}\end{array}$ & $\begin{array}{l}\geq \\
\text { 忘}\end{array}$ & 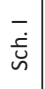 & $\begin{array}{l}\geq \\
i \\
i \\
\end{array}$ & $\begin{array}{l}\geq \\
\geq \\
\dot{i} \\
\dot{\omega}\end{array}$ & $\begin{array}{l}\geq \\
\vdots \\
\dot{E} \\
\end{array}$ & 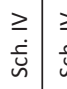 & 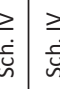 & 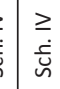 \\
\hline 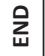 & & & $\stackrel{0}{3}$ & & & $\stackrel{0}{3}$ & ڤ & ڤ & & & & & & & & & & & & & & & ڤั) \\
\hline z & u & u & u & u & u & u & z & z & u & u & u & u & $\cup$ & u & u & u & 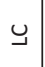 & u & u & u & u & $\begin{array}{c}4 \\
y\end{array}$ & u \\
\hline 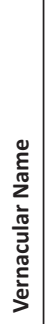 & 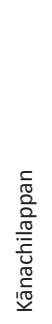 & 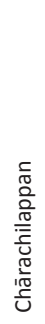 & 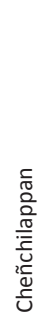 & 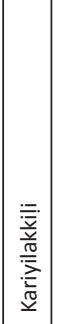 & 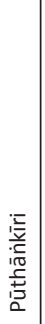 & 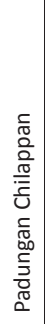 & 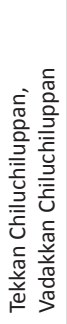 & 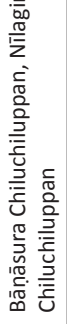 & 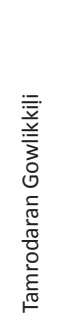 & 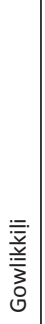 & 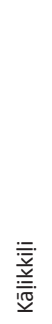 & 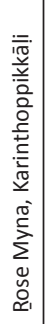 & 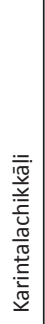 & 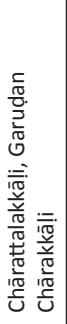 & 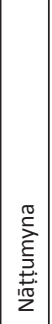 & 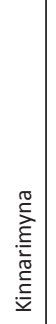 & 离 & 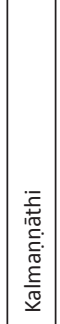 & 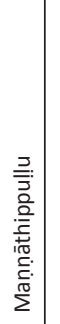 & 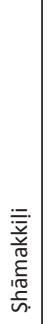 & 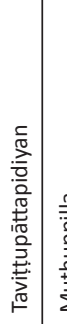 & 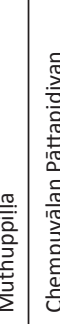 & 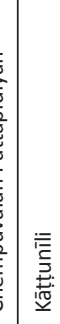 \\
\hline 冚 & 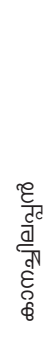 & 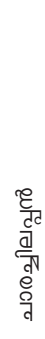 & 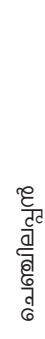 & 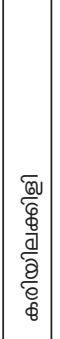 & 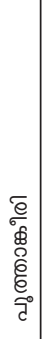 & 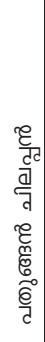 & 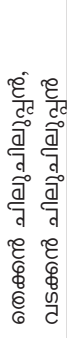 & 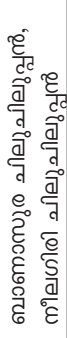 & 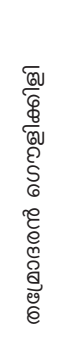 & 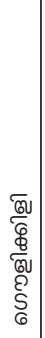 & 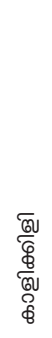 & 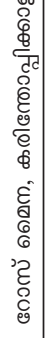 & 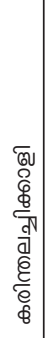 & 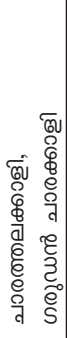 & $\begin{array}{l} \\
\\
\varepsilon \\
6 \\
6 \\
6 \\
0 \\
0 \\
\stackrel{0}{0} \\
\varepsilon\end{array}$ & 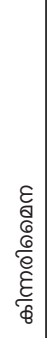 & 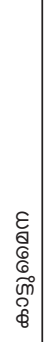 & 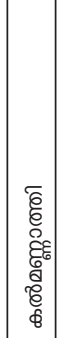 & 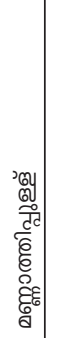 & $\begin{array}{l}\widetilde{\omega} \\
\frac{\Phi}{\Phi} \\
\bar{\Phi} \\
\text { वृ. }\end{array}$ & 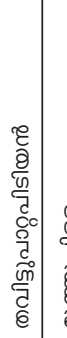 & 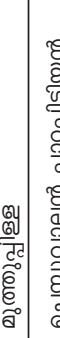 & 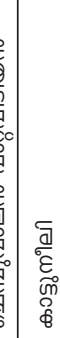 \\
\hline
\end{tabular}

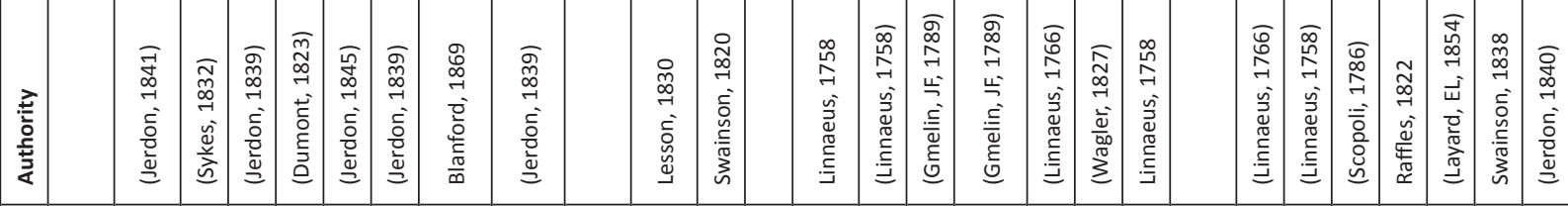

\begin{tabular}{|c|c|c|c|c|c|c|c|c|c|c|c|c|c|c|c|c|c|c|c|c|c|c|c|c|c|c|}
\hline 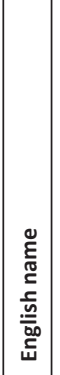 & 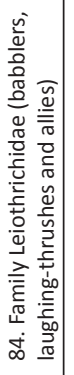 & 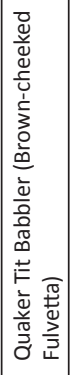 & 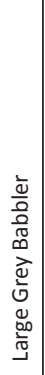 & 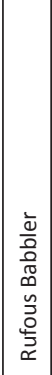 & 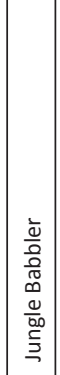 & 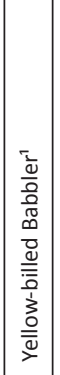 & 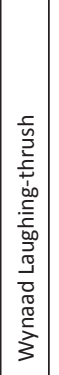 & 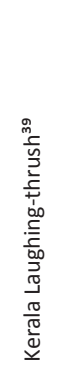 & 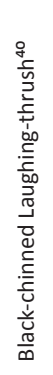 & 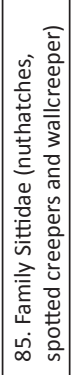 & 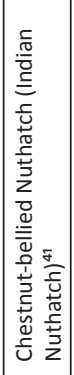 & 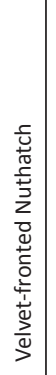 & 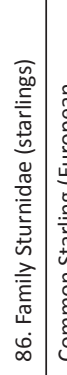 & 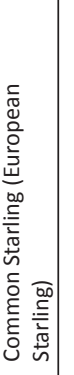 & 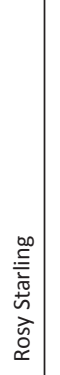 & 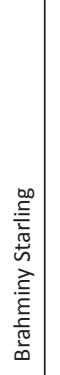 & 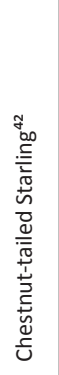 & 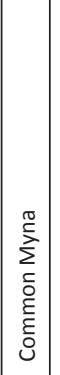 & $\mid$ & 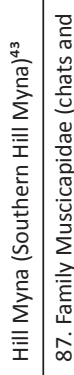 & 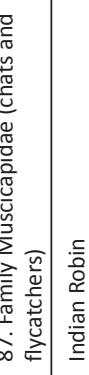 & 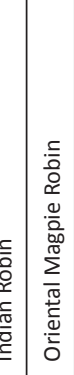 & 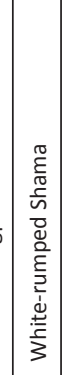 & 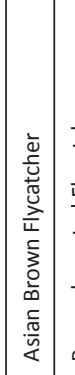 & | & 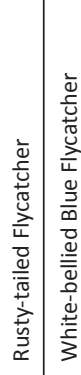 \\
\hline & & 守 & & 竎 & 夠 & 吕 & ริ & 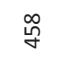 & 多 & & 导 & 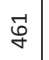 & & శ్ & 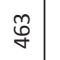 & 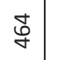 & 強 & $\begin{array}{l}\text { bo } \\
\vdots\end{array}$ & f & f & ơ & 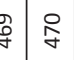 & $|\vec{q}|$ & $\mid \begin{array}{c}\tilde{f} \\
\end{array}$ & $\stackrel{m}{f}$ & $\begin{array}{l}\vec{g} \\
\dot{q}\end{array}$ \\
\hline
\end{tabular}




\begin{tabular}{|c|c|c|c|c|c|c|c|c|c|c|c|c|c|c|c|c|c|c|c|c|c|c|c|c|c|}
\hline 岁 & & & & & & & & & & & & & & & & & & & & & & & & & \\
\hline$\widehat{\hat{a}}$ & $\begin{array}{l}\geq \\
\dot{i} \\
\dot{\omega}\end{array}$ & $\begin{array}{l}\geq \\
\stackrel{\dot{\nu}}{\dot{\omega}}\end{array}$ & $\begin{array}{l}\geq \\
\text { 离 }\end{array}$ & $\begin{array}{l}\geq \\
\dot{.} \\
\dot{\omega}\end{array}$ & $\begin{array}{l}\geq \\
\stackrel{\dot{\nu}}{\dot{\omega}}\end{array}$ & 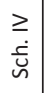 & $\begin{array}{l}\geq \\
\geq \\
\dot{\bar{n}}\end{array}$ & $\begin{array}{l}\geq \\
\text { 离 }\end{array}$ & $\begin{array}{l}\geq \\
\text { i } \\
\text { in }\end{array}$ & $\begin{array}{l}\geq \\
\stackrel{\vec{\Delta}}{\mathbf{n}}\end{array}$ & 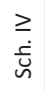 & $\begin{array}{l}\geq \\
\text { 它 }\end{array}$ & $\begin{array}{l}\geq \\
\text { 它 }\end{array}$ & $\begin{array}{l}\geq \\
\text { : } \\
\bar{\omega}\end{array}$ & $\begin{array}{l}\geq \\
\dot{\vec{j}} \\
\text { 足 }\end{array}$ & $\mid \begin{array}{l}\geq \\
\dot{\vec{L}} \\
\tilde{\omega}\end{array}$ & $\begin{array}{l}\geq \\
\text { 离 } \\
\text {. }\end{array}$ & $\begin{array}{l}\geq \\
\text {. } \\
\text { n. }\end{array}$ & $\begin{array}{l}\geq \\
\text {. } \\
\text { n }\end{array}$ & $\begin{array}{l}\geq \\
\geq \\
\text { 它 } \\
\omega\end{array}$ & $\begin{array}{l}\geq \\
\geq \\
\underline{\dot{L}} \\
\omega\end{array}$ & $\begin{array}{l}\geq \\
\dot{\vec{L}} \\
\end{array}$ & $\begin{array}{l}\geq \\
\text { 悹 }\end{array}$ & $\begin{array}{l}\geq \\
\text { 离 }\end{array}$ & $\begin{array}{l}\geq \\
\overline{5} \\
\bar{v}\end{array}$ \\
\hline$\sum_{\mathrm{u}}^{2}$ & & & & $\varliminf_{3}^{0}$ & ${ }_{3}^{\circ}$ & & & & & & & & $\stackrel{0}{3}^{\circ}$ & & & & & & & & & & & & \\
\hline$\underline{\underline{z}}$ & צ & צ & y & ह & z & u & צ & u & 3 & y & u & צ & 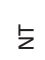 & U & u & u & צ & u & u & צ & u & y & u & y & $\frac{\vec{z}}{z}$ \\
\hline
\end{tabular}

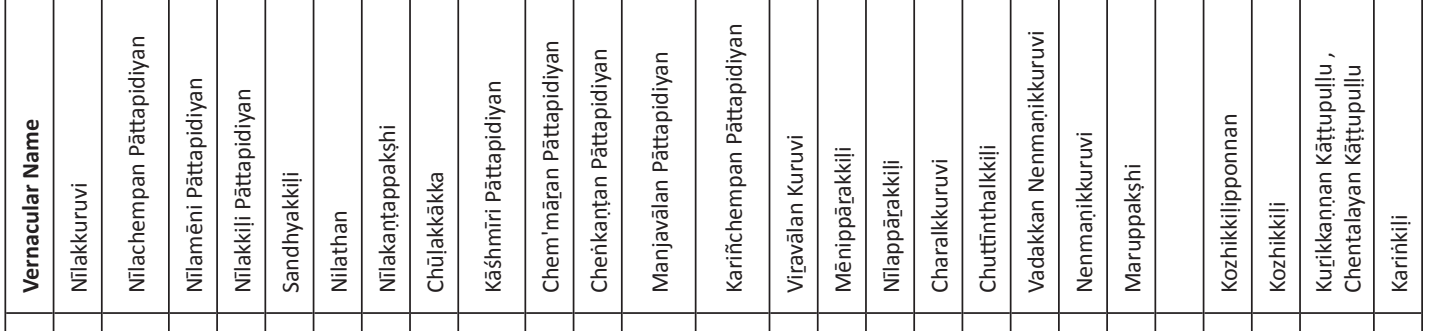

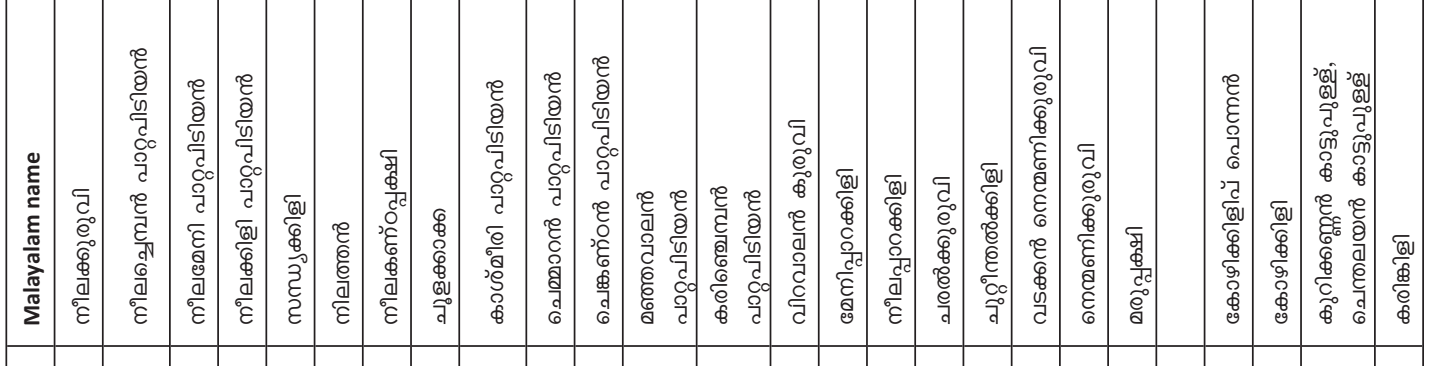

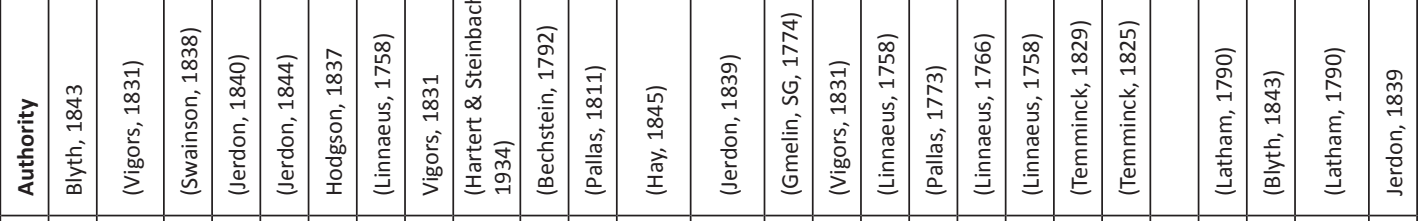

\begin{tabular}{|c|c|c|c|c|c|c|c|c|c|c|c|c|c|c|c|c|c|c|c|c|c|c|c|}
\hline & 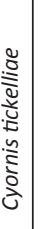 & 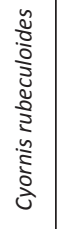 & 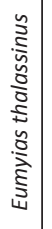 & 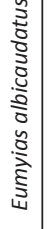 & 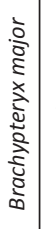 & 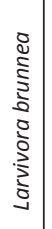 & 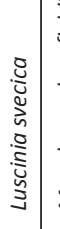 & 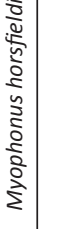 & 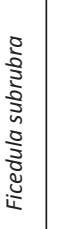 & 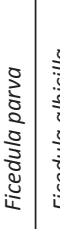 & 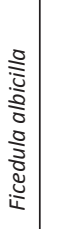 & 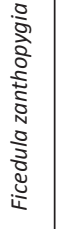 & 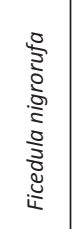 & 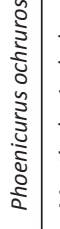 & 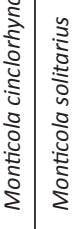 & 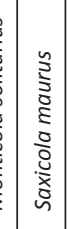 & 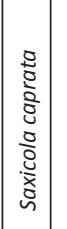 & 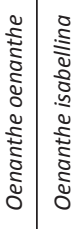 & 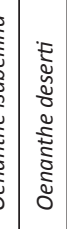 & & 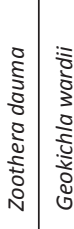 & 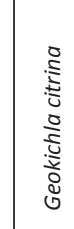 & \\
\hline 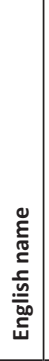 & 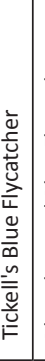 & 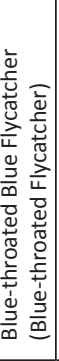 & 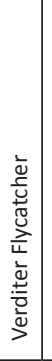 & 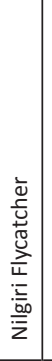 & 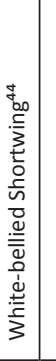 & 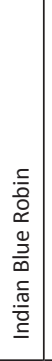 & | & 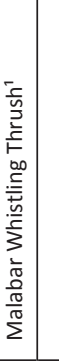 & 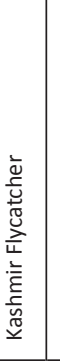 & 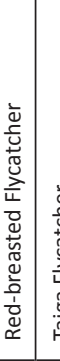 & 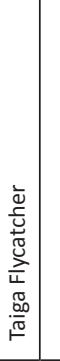 & 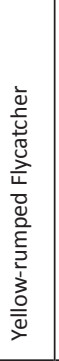 & 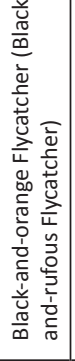 & 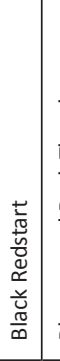 & 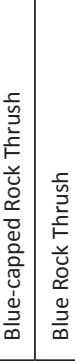 & 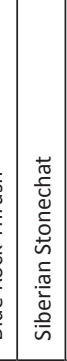 & 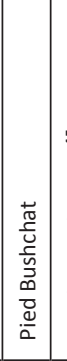 & & 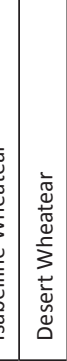 & 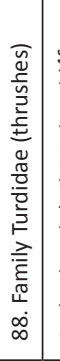 & 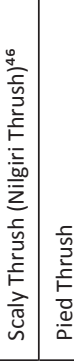 & 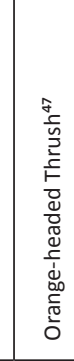 & \\
\hline & $\mathscr{L}^{\circ}$ & $\hat{f}$ & $\hat{f}$ & $\stackrel{\infty}{\&}$ & 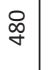 & $\begin{array}{ll}\vec{g} \\
\stackrel{\sigma}{a}\end{array}$ & 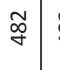 & $\begin{array}{l}\stackrel{\infty}{\leftrightarrow} \\
\stackrel{\sigma}{\sigma}\end{array}$ & 㐫 & 岁 & 这 & क्ष & $\begin{array}{l}\infty \\
\stackrel{\infty}{a} \\
\stackrel{1}{2}\end{array}$ & 搼 & 守 & హ్ & 啇 & 孛 & $\begin{array}{l}3 \\
\end{array}$ & & \begin{tabular}{l|l}
$\stackrel{\infty}{g}$ & $\stackrel{\infty}{g}$
\end{tabular} & g & ìn \\
\hline
\end{tabular}


${ }^{1}$ Endemic to Peninsular India.

${ }^{2}$ Regional population considered naturalised in origin.

${ }^{3}$ Mannar \& Ambika (2014)

${ }^{4}$ Endemic race of Western Ghats Grey-fronted Green Pigeon T. p. affinis sometimes accorded species status (Birdlife International 2014, Clements et al. 2014, Gill \&

Donskar 2014).

${ }^{5}$ Endemic race of Western Ghats Nilgiri Imperial Pigeon D. b. cuprea sometimes accorded species status (Birdlife International 2014).

${ }^{6}$ Endemic to Western Ghats \& Sri Lanka.

${ }^{7}$ Regionally occurring wintering race Fork-tailed Drongo Cuckoo S. I. dicruroides sometimes accorded species status (Birdlife International 2014; Clements et al. 2014; Gill \& Donskar 2014).

${ }^{8}$ Praveen et al. (2013)

9 Praveen et al. (2015)

${ }^{10}$ Regionally occurring passage birds are of race Persian Shearwater $P$. b. persicus and is sometimes accorded species status (Birdlife International 2014; Clements et al. 2014; Gill \& Donskar 2014).

${ }^{11}$ Birdlife International (2014) splits African Woollyneck C. microscelis of Africa from this species and then lists Asian Woollyneck C. episcopus as Vulnerable.

${ }^{12}$ Gandhe (2014)

${ }^{13}$ Historical.

${ }^{14}$ Sreenivasan (2015)

${ }^{15}$ Historical. Recent sight records not sufficiently corraborated.

${ }^{16}$ Historical. Recent photographic records unconfirmed.

17 Bhat (2014)

18 Includes two regionally wintering subspecies - Heuglin's Gull L. f. heuglini \& Steppe Gull L. f. barabensis.

${ }^{19}$ Endemic race of Western Ghats and Sri Lanka, N. n. kelaarti, sometimes accorded species status and named Legge's Hawk Eagle (Clements et al. 2014; Gill \&

Donskar 2014).

${ }^{20}$ Endemic race of Western Ghats ripleyi is sometimes considered under race assimilis as an independent species named Sri Lanka Bay Owl (Clements et al. 2014, Gill \& Donskar 2014).

${ }^{21}$ Formal publication of the record is under way.

${ }^{22} \mathrm{E}$. Indian race $O$. b. lettia is sometimes considered a different species and takes the name Collared Scops Owl while the nominate is called Indian Scops Owl (Birdlife International 2014; Clements et al. 2014; Gill \& Donskar 2014).

${ }^{23}$ Birdlife International (2014) accords species status to the African race F. c. ruficollis that takes the name Red-necked Falcon and the regionally occurring nominate

is called Red-headed Falcon and the same is listed as Near-Threatened.

${ }^{24}$ Includes distinct resident subspecies Shahin Falcon F. p. perigrinator.

25 Regional population considered naturalised in origin (Rasmussen \& Anderton 2012).

${ }^{26}$ Himalayan race $P$. f. speciosus is sometimes considered a different species and takes the name Scarlet Minivet while the nominate is called Orange Minivet

(Clements et al. 2014; Gill \& Donskar 2014).

${ }^{27}$ Endemic race of Western Ghats Malabar Woodshrike T. virgatus sylvicola sometimes accorded species status (Clements et al. 2014; Gill \& Donskar 2014).

${ }^{28}$ Includes distinct subspecies Philippine Shrike L. c. lucionensis.

${ }^{29}$ Regionally occurring Indian Jungle Crow C. m. culminatus is sometimes considered a distinct species (Gill \& Donskar 2014).

${ }^{30}$ Races of northeastern India D. c. minullum and Andamans D. c. virescens are sometimes considered different species and hence the Western Ghat endemic

nominate race takes the name Nilgiri Flowerpecker while the name Plain Flowerpecker is used for minullum (Clements et al. 2014; Gill \& Donskar 2014).

${ }^{31}$ George (2015)

32 Endemic race of Western Ghats travancoreensis is sometimes considered under race aplonotus as an independent species named Indian Black-lored Tit/Indian Tit (Clements et al. 2014, Gill \& Donskar 2014).

${ }^{33}$ Endemic to west coast of India.

${ }^{34}$ Endemic race of Western Ghats and Sri Lanka, H. t. domicola, sometimes accorded species status and named Hill Swallow (Clements et al. 2014; Gill \& Donskar 2014).

${ }^{35}$ Endemic race of Western Ghats and Sri Lanka, H. I. ganeesa, sometimes accorded species status and named Square-tailed Bulbul (Clements et al. 2014; Gill \& Donskar 2014)

${ }^{36}$ Endemic race of Western Ghats, Flame-throated Bulbul P. m. gularis, sometimes accorded species status (Clements et al. 2014; Gill \& Donskar 2014).

${ }^{37}$ Regionally occurring wintering/passage birds are of race althaea sometimes accorded species status and named Hume's Whitethroat (Birdlife International 2014;

Clements et al. 2014; Gill \& Donskar 2014).

${ }^{38}$ Endemic to Western Ghats \& Sri Lanka. Includes distinct subspecies Bourdillon's Babbler R. a. bourdilloni.

${ }^{39}$ Endemic to Western Ghats south of Palakkad gap. Sometimes treated as two species - Palani Laughingthrush S. fairbanki \& Travancore Laughingthrush S. meridionalis (Praveen \& Nameer 2012).

${ }^{40}$ Endemic to Western Ghats north of Palakkad gap up to Coorg. Sometimes treated as two species - Banasura Laughingthrush S. jerdoni \& Nilgiri Laughingthrush S. cachinnans (Praveen \& Nameer 2012).

${ }^{41}$ Himalayan race S. c. cinnamoventris is sometimes considered a different species and takes the name Chestnut-bellied Nuthatch while the nominate is called Indian Nuthatch (Clements et al. 2014; Gill \& Donskar 2014).

42 Includes distinct endemic race Malabar Starling S. m. blythi, sometimes accorded species status (Clements et al. 2014; Gill \& Donskar 2014).

${ }^{43}$ Endemic race of Western Ghats and Sri Lanka, G. r. indica, sometimes accorded species status and named Southern Hill Myna (Clements et al. 2014; Gill \& Donskar 2014).

${ }^{44}$ Includes two endemic subspecies, Nilgiri Shortwing/Nilgiri Blue Robin B. m. major found in the Western Ghats north of Palakkad gap till Bababudan Hills and White-bellied Shortwing/White-bellied Blue Robin B. m. albiventris found south of the gap. Based on molecular studies, Robin et al. (2010) proposes to elevate the two races to species level. These are treated as two distinct species by Birdlife International (2014), Clements et al. (2014) and Gill \& Donskar (2014) and both are classified as Endangered.

Includes two endemic subspecies, Nilgiri Shortwing/Nilgiri Blue Robin B. m. major found in the Western Ghats north of Palakkad gap till Bababudan Hills and White-bellied Shortwing/White-bellied Blue Robin B.m.albiventirs found south of the gap. These are treated as two distinct species by Birdlife International (2014), Clements et al. (2014) and Gill \& Donskar (2014) and both are classified as Endangered.

45 Damle \& Inskipp (2014)

${ }^{46}$ The endemic race of Western Ghats, Z. d. neilgherriensis, sometimes accorded species status and called Nilgiri Thrush (Gill \& Donskar 2014).

${ }^{47}$ Includes distinct subspecies White-throated Ground Thursh G. c. cyanota.

${ }^{48}$ Endemic to Peninsular India. Includes distinct migratory subspecies T. s. nigropileus \& resident T. s. bourdilloni apart from nominate.

${ }^{49}$ Karuthedathu et al. (2015) 


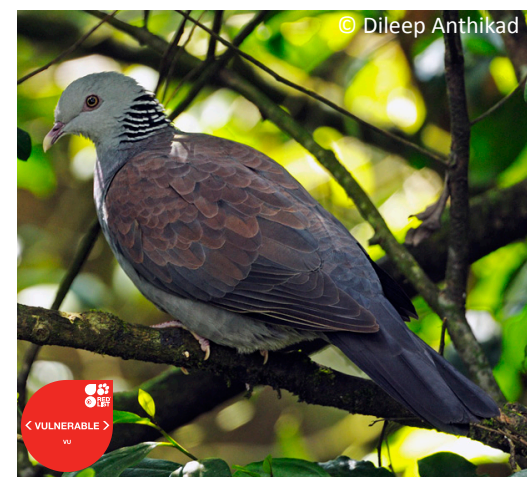

Image 1. Nilgiri Wood-Pigeon

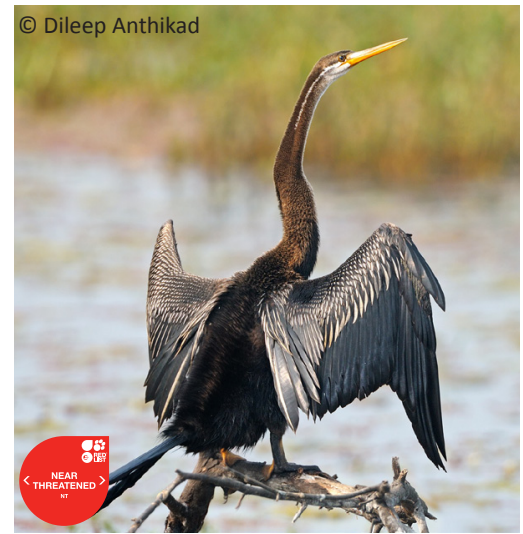

Image 4. Oriental Darter

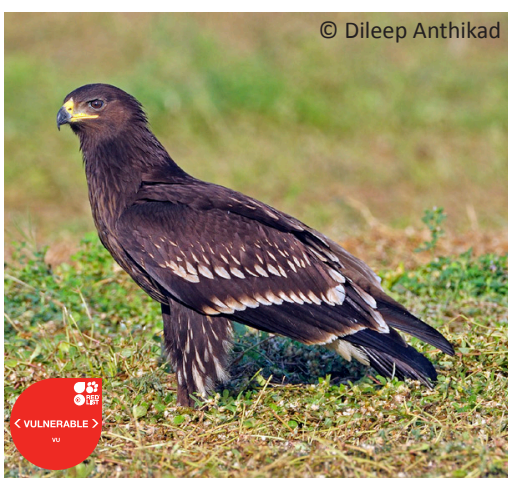

Image 7. Greater Spotted Eagle

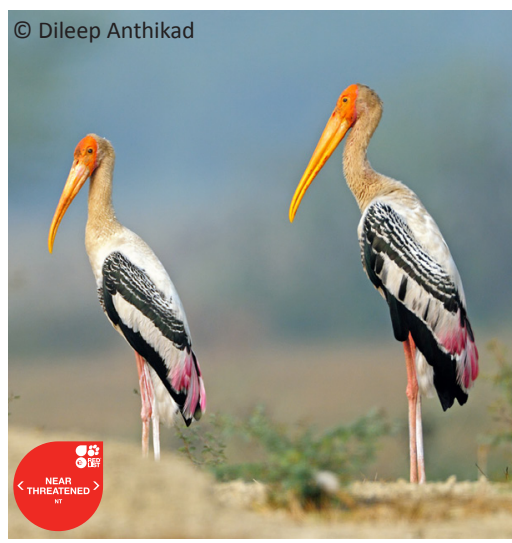

Image 2. Painted Stork

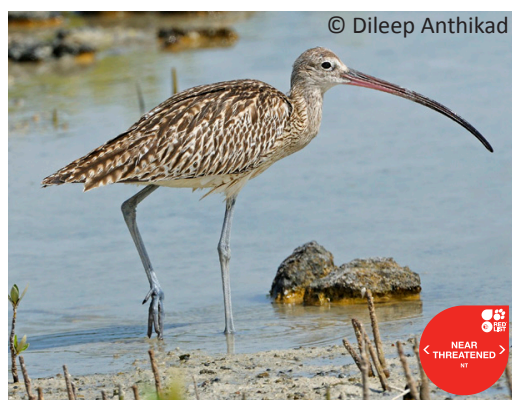

Image 5. Eurasian Curlew

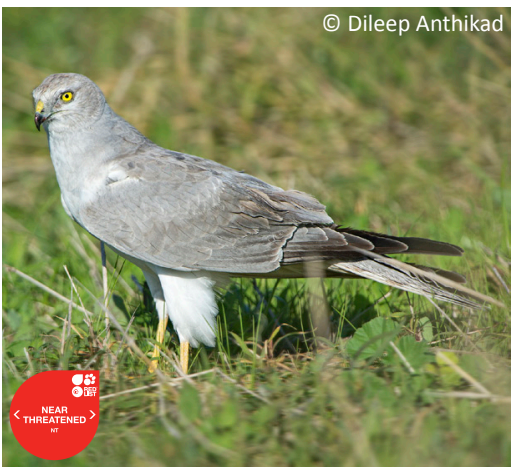

Image 8. Pallid Harrier

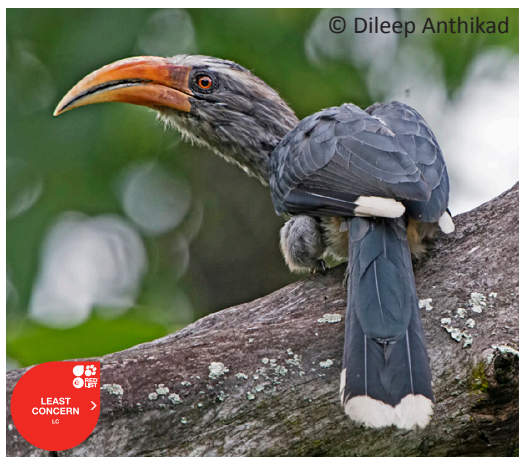

Image 10. Malabar Grey Hornbill

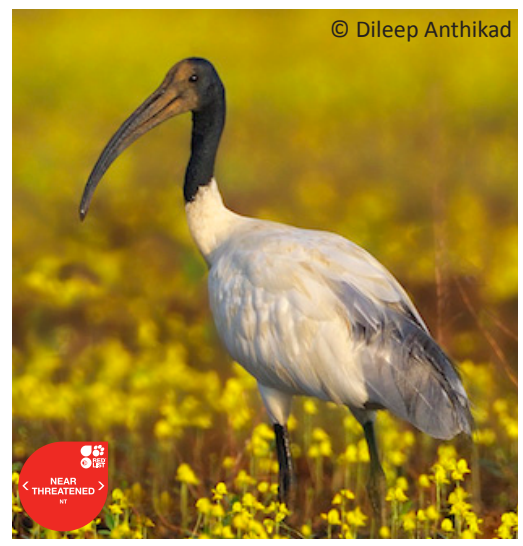

Image 3. Black-headed Ibis

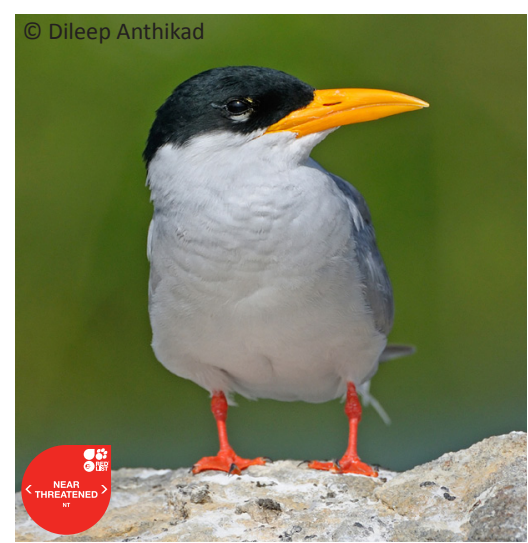

Image 6. River Tern

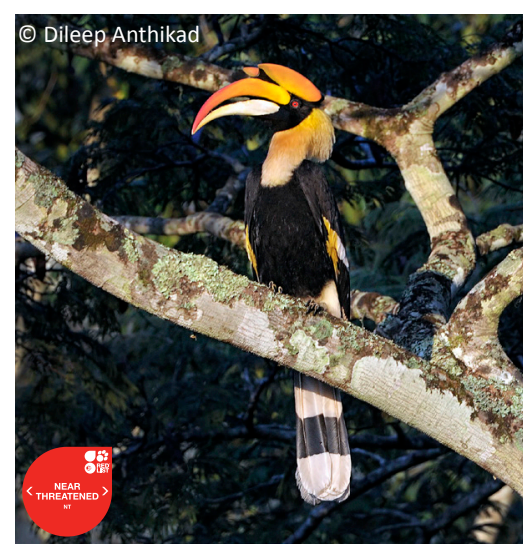

Image 9. Great Indian Hornbill 


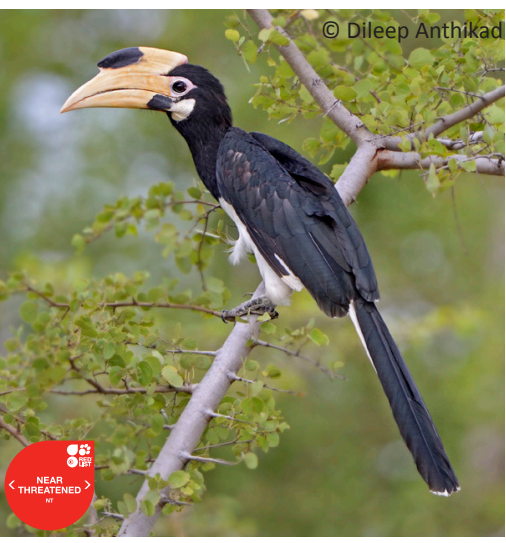

Image 11. Malabar Pied Hornbill

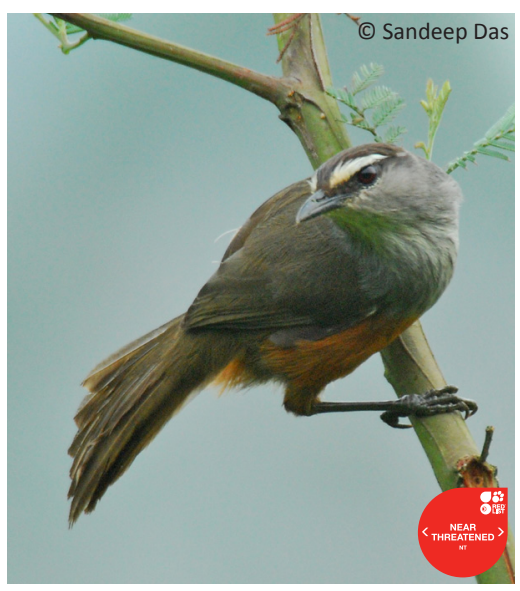

Image 14. Kerala Laughing-thrush (Palani Laughing-thrush

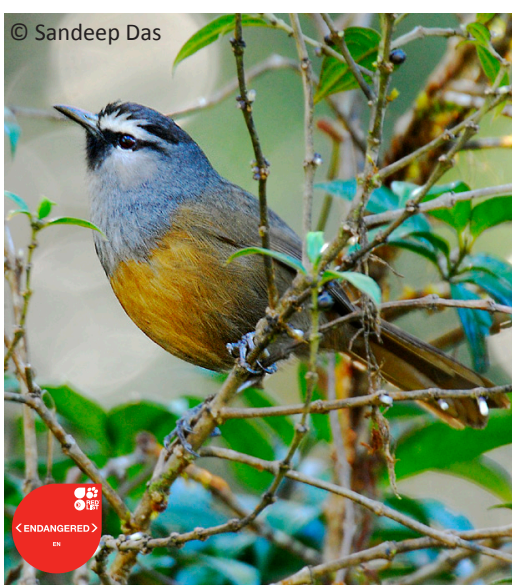

Image 17. Black-chinned Laughing-thrush (Banasura Laughing-thrush)

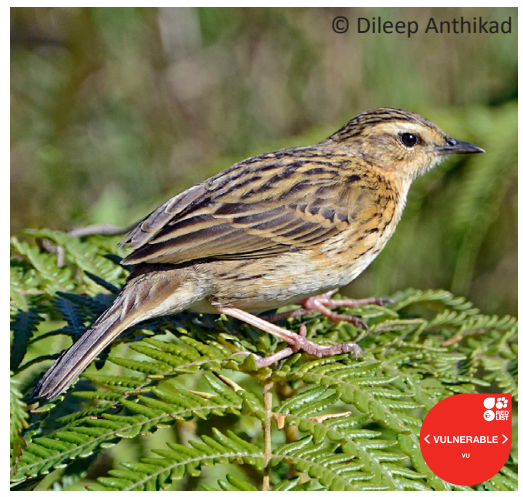

Image 12. Nilgiri Pipit

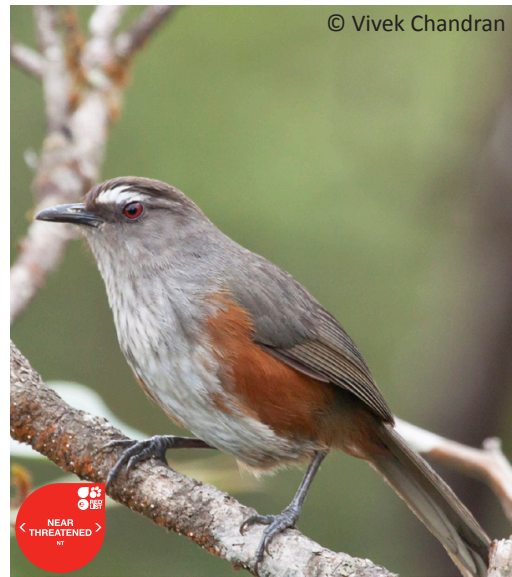

Image 15. Kerala Laughing-thrush

(Travancore Laughing-thrush)

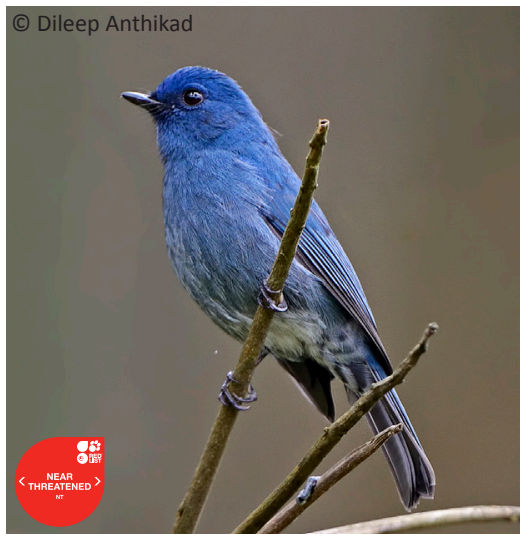

Image 18. Nilgiri Flycatcher

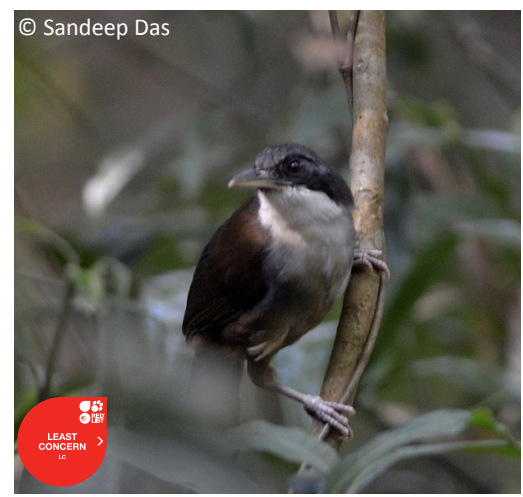

Image 13. Wynaad Laughingthrush

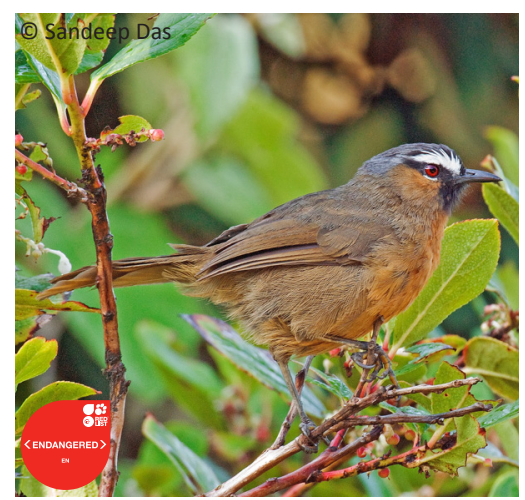

Image 16. Black-chinned Laughing-thrush (Nilgiri Laughing-thrush)

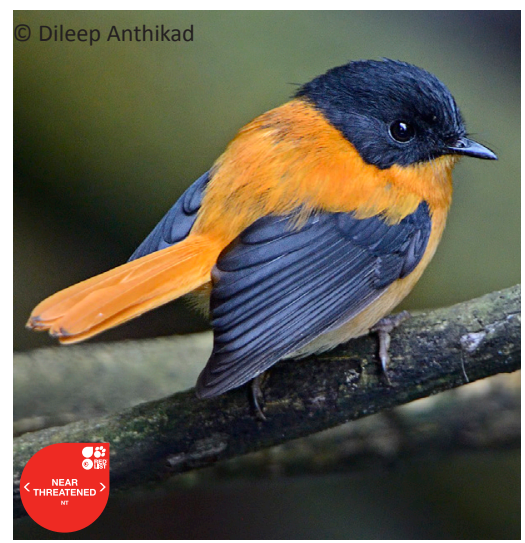

Image 19. Black-and-orange Flycatcher 
Davidson, S. Manickam, V. Rao \& M. Vineeth (2015). A compilation of frigatebird sightings from 2014, including Christmas Island Frigatebird Fregata andrewsi. Indian BIRDS 10(5): 115-118.

Mannar, H. \& D.S. Ambika (2014). Snapshot sightings: Red Turtle Dove from Haripad, Kerala. Indian BIRDS 9(5\&6): 168A.

Neelakantan, K.K. (1984). Keralathile Pakshikal. $2^{\text {nd }}$ Edition. Kerala Sahitya Academy. Trichur

Neelakantan, K. K., C. Sashikumar \& R. Venugopalan (1993). A Book of Kerala Birds - Part 1. World Wide Fund for Nature-India. Kerala State Committee, Trivandrum, 146pp.

Praveen, J. \& P.O. Nameer (2013). Strophocincla Laughingthrushes of south India: a case for allopatric speciation and impact on their conservation. Journal of the Bombay Natural History Society 109(1\&2): 46-52 (2012)

Praveen, J., R. Jayapal \& A. Pittie (2013). Notes on Indian rarities - 1: Seabirds. Indian Birds 8(5): 113-125

Praveen, J., R. Jayapal \& A. Pittie (2014). Checklist of the birds of South Asia (Ver. 1). Website: http://www.indianbirds.in/resources/. [Date of publication: 26 December 2014.]
Praveen, J. \& S.P. Narayanan (2014). Additions to the checklist of birds of Kerala. Malabar Trogon 11: (1-3) 2-12 (2013).

Praveen E.S., S. Das \& K.G. Dilip (2015). Sighting of Short-tailed Shearwater Ardenna tenuirostris and Wedge-tailed Shearwater Ardenna pacifica from the Arabian Sea, off Ponnani, Kerala. Indian BIRDS 10(1): 26-27.

Rasmussen, P.C. \& J.C. Anderton (2012). Birds of South Asia: the Ripley Guide: Attributes and Status - 2. Smithsonian Institution and Lynx Edicions, Washington, D.C. and Barcelona, 683pp.

Robin, V.V., A. Sinha \& U. Ramakrishnan (2010). Ancient geographical gaps and paleo-climate shape the phylogeography of an endemic bird in the Sky Islands of southern India. PLOS ONE 5(10): e13321; http://dx.doi.org/10.1371/journal.pone.0013321

Sashikumar, C., J. Praveen, M.J. Palot \& P.O. Nameer (2010). A checklist of birds of Kerala. Malabar Trogon 7(3): 2-13 (2009).

Sashikumar, C., J. Praveen, M.J. Palot \& P.O. Nameer (2011). Birds of Kerala: Status and Distribution. DC Books, Kottayam, Kerala, 835pp. Sreenivasan, P.P. (2015). Snapshot sightings: Red Knot at Chavakkad, Kerala. Indian BIRDS 10(1): 28A 